\title{
Total Saponins of Panax Notoginseng Exhibit Neuroprotection by The Activation of Akt/mTOR Pathway in Vitro and in Vivo
}

\section{Dong-Ping Wu}

Jinan University

Yu-Wei Pan

Jinan University

Hua-Feng Liang

Jinan University

Gen-Yun Tang

Jinan university

Ming Chen

Jinan University

Guan-Ping Liu

Jinan university

Li-Li Zhang

Jinan University

Chun-Lin Fan

Jinan University

Lei Shi

Jinan University

Wen-Cai Ye

Jinan University

ManMei Li ( $\nabla$ jnulimanmei1209@126.com )

Jinan University

\section{Research}

Keywords: Total saponins of Panax notoginseng (Burkill) F.H.Chen, oxygen-glucose deprivation and reoxygenation, neuroprotection, ischemia-reperfusion, Akt/mTOR pathway

Posted Date: November 9th, 2020

DOl: https://doi.org/10.21203/rs.3.rs-102342/v1 
License: (c) (i) This work is licensed under a Creative Commons Attribution 4.0 International License. Read Full License 
Total saponins of Panax notoginseng exhibit neuroprotection by the activation of Akt/mTOR pathway in vitro and in vivo

Dong-Ping Wu ${ }^{1 \S}$, Yu-Wei Pan ${ }^{2,3 \S}$, Hua-Feng Liang ${ }^{1}$, Gen-Yun Tang ${ }^{2}$, Ming Chen ${ }^{4}$, Guan-Ping Liu ${ }^{4}$, Li-Li Zhang ${ }^{1}$, Chun-Lin Fan ${ }^{1}$, Lei Shi ${ }^{2 *}$, Wen-Cai Ye ${ }^{1 *}$, Man-Mei $\mathbf{L i}{ }^{*}$

${ }^{1}$ Institute of Traditional Chinese Medicine and Natural Products, College of Pharmacy, Jinan University, Guangzhou, Guangdong 510632, China

${ }^{2}$ JNU-HKUST Joint Laboratory for Neuroscience and Innovative Drug Research, Jinan University, Guangzhou, Guangdong 510632, China.

${ }^{3}$ Guangzhou Tianhe Traditional Chinese Medicine Hospital, Guangzhou, Guangdong 510632, China

${ }^{4}$ ZhongHeng Group Guangxi Wuzhou pharmaceutical Company, Guangxi, Wuzhou 543000, China

\section{Author Contributions}

${ }^{\S}$ Dong-Ping Wu and Yu-Wei Pan contributed equally to this study,

*Corresponding authors

Man-Mei Li, College of Pharmacy, Jinan University, Guangzhou 510632, China Phone: (86) 20 85221646, Fax: (86) 20 85221559, E-mail: limanmei@hotmail.com Wen-Cai Ye, College of Pharmacy, Jinan University, Guangzhou 510632, China Phone: (86) 20 85220936, Fax: (86) 20 85221559, E-mail: chywc@ aliyun.com Lei Shi, JNU-HKUST Joint Laboratory for Neuroscience and Innovative Drug Research, Jinan University, Guangzhou, Guangdong 510632, China Phone: (86) 20 85222120, Fax: (86) 20 85222112, E-mail: sophielshi80@gmail.com

\section{Email addresses:}

MML: jnulimanmei1209@126.com

WCY: chywc@aliyun.com

LS: sophielshi80@gmail.com

DPW: 1448473002@qq.com 
YWP: pywyvette@foxmail.com

HFL: 834301227@qq.com

GT: tanggenyun@foxmail.com

MC: cmhk1898@163.com

GPL:1gp3808@126.com

LLZ: 757741498@qq.com

CLF: jnuchunlin@163.com 


\begin{abstract}
:
Background: Panax notoginseng (Burkill) F.H.Chen is a traditional Chinese medicine. The present study reports the potential therapeutic effect of total saponins of Panax notoginseng (Burkill) F.H.Chen (TSPN) on ischemic stroke and investigates the underlying mechanisms. To reveal the neuroprotective effect of TSPN on cerebral ischemia-reperfusion injury and the underlying mechanisms.
\end{abstract}

Methods: Oxygen-glucose deprivation/reoxygenation (OGD/R) of cultured cortical neurons was used as a model of neuronal injury. The neuroprotective effect of TSPN was evaluated by 3-(4, 5-dimethylthiazol-2-yl)-2,5-diphenyl tetrazolium bromide (MTT) assay, flow cytometry and Live/Dead cell assay. The morphology of dendrites was detected by immunofluorescence. Rat middle cerebral artery occlusion (MCAO) was used as a model of cerebral ischemia-reperfusion. The neuroprotective effect of TSPN was evaluated by neurological score, tail hang test, TTC staining, and Nissl staining. Western blot analysis, immunohistochemistry or immunofluorescence was used to measure the changes in Akt/mTOR signaling pathway.

Results: MTT showed that TSPN had a rescue effect on cortical neurons after OGD/Rtreated. Flow cytometry and Live/Dead cell assay indicated that TSPN decreased neuronal apoptosis, and immunofluorescence showed that TSPN restored dendrite morphology of the damaged neurons. Moreover, TSPN down-regulated the expressions of cleaved-Caspase-3 and LC3B-II/LC3B-I, whereas up-regulated the levels of phosphorylated (p)- Akt and p-mTOR. In MCAO model, TSPN rescued defective neurological behavior and reduced infarct volume. Moreover, the expressions of Beclin-1 and LC3B in cerebral ischemic penumbra were down-regulated after the treatment of TSPN, whereas p-mTOR level was up-regulated.

Conclusion: TSPN show neuroprotective effects against OGD/R-induced cortical neuronal damage and exhibit potential therapeutic effects on cerebral ischemia in rat MCAO model. Up-regulation of mTOR pathway and inhibition of the autophagic pathway could be the mechanisms that underlie the action of TSPN.

Key words: Total saponins of Panax notoginseng (Burkill) F.H.Chen, oxygen-glucose deprivation and re-oxygenation, neuroprotection, ischemia-reperfusion, Akt/mTOR pathway. 


\section{Background}

Stroke is generally a cerebrovascular disease, with an expected increase of 10.3 million per year, represents a major threat to human health, and represents a great burden on society [1]. Stroke includes ischemic and hemorrhagic strokes. The former stroke is responsible for 60 to $70 \%$ of all stroke cases. Stroke caused by cerebral ischemia can lead to cerebral infarction, leading to cerebral ischemia and hypoxia, with reduced local cerebral blood flow or interruption of blood supply [2]. The oxygenglucose deprivation/reoxygenation (OGD/R) model is commonly used in laboratories as a cytological model in hypoxia. Cultures lack glucose and oxygen, along with the physical or chemical mechanisms that feed oxygen around the cells, replacing essential nutrients. Meanwhile, the middle cerebral artery occlusion (MCAO) model makes animals suffer from ischemic stroke, as well as in patients with clinical stroke. Therefore, MCAO is a classic model for assessing the effectiveness of neuroprotective drugs or other treatment methods, analyzing the pathological mechanisms of cerebral ischemia [3].

Ischemic stroke is a disease that causes irreversible damage to the central area of cerebral ischemia, which involves abnormal blood flow, an imbalance of excitatory toxic ions by the release of glutamic acid, destruction of the blood-brain barrier, and a series of complex pathological processes, along with oxidative stress, inflammatory response, necrosis, apoptosis, and autophagy [4]. However, Ischemic penumbra is a potential area of clinical stroke treatment, which is damaged brain tissue around the central ischemic area that can be recovered [5].

Autophagy is an energy-dependent process related to the mechanism of cell death and is an essential step for cell growth, development, and homeostasis, which enables the materials in cells to maintain a relatively balanced state in synthesis, degradation, and circulation. A great deal of previous research has been reported the relationships between autophagy and ischemic. Some studies showed that intermediate autophagy alleviates brain damage [6], while others reported that inhibiting autophagy mitigates brain damage [7-9]. Therefore, it has been proposed that the time and degree of autophagy may influence cerebral ischemia and whether the process of autophagy is 
impaired [10]. This supports the evidence that the prolongation of ischemia leads to autophagy overactivation, diverting the effects of the autophagy pathway from protecting brain tissue to damage [11].

In clinical, it is universal to cure ischemic stroke using Chinese herbal medicines and standardized preparations of Chinese herbal medicines. Total saponins of Panax notoginseng (Burkill) F.H.Chen (TSPN) are the main ingredient of Xueshuantong (XST) for injection (lyophilized), and they are particularly useful in treating vascular and cardiovascular diseases, such as ischemic stroke, acute cerebral hemorrhage, and heart disease in China or other oriental countries $[12,13]$. Recently, more and more studies have been showed that TSPN have neuroprotective effects as well as antioxidant properties [14-16]. TSPN have been shown to improve cerebral ischemia and protect brain tissue from a stroke. Potential mechanisms include inhibiting apoptosis, promoting nerve cell regeneration, scavenging oxygen free radicals, anti-inflammation, anti-thrombosis, inhibiting excitatory amino acid toxicity, and $\mathrm{Ca}^{2+}$ influx [17-22]. Moreover, A previously published study reported that TSPN altered the expression of autophagy-related proteins in a rat model of cisplatin-induced kidney injury [23]. However, whether TSPN affect the autophagy pathway during cerebral ischemia has remained unidentified. In the literature, we investigate the ability of TSPN on inhibiting autophagy not only on oxygen-glucose deprivation/reperfusion (OGD/R) neurons, an in vitro model to simulate cerebral ischemia but also on MCAO rats, an in vivo model to mimic stroke.

\section{Material and methods}

\section{Chemicals and reagents}

Acetonitrile and methanol (HPLC grade) were purchased from Merck (Darmstadt, Germany). The water used was purified by Milli-Q system (Millipore, USA). Reference compounds, namely notoginsenoside $\mathrm{R} 1$, ginsenoside $\mathrm{Rg} 1, \mathrm{Re}, \mathrm{Rb} 1$ and $\mathrm{Rd}$ were extracted from Jinan University (Guangzhou, China). Total saponins of Panax notoginseng (TSPN) were provided by Guangxi Wuzhou Pharmaceutical Co, Ltd (Guangxi, China. batch number 16101515). Neurobasal culture medium, B27 and D- 
Hank's solution were purchased from Gibco (Life Technologies, Carlsbad, CA, USA); Poly-L-Lysine was from Sigma-Aldrich (St. Louis, MO, USA); Rabbit monoclonal antiBeclin-1, anti-LC3B, anti-cleaved-Caspase-3, anti-AKT, anti-p-AKT, anti-mTOR, and anti-p-mTOR were from Cell Signaling Technology (Danvers, MA, USA); 3- (4,5dimethylthiazol-2-yl) -2,5-diphenyl tetrazolium bromide (MTT) and dimethyl sulfoxide (DMSO) were from Sigma-Aldrich. Monofilament nylon thread was purchased from Beijing Cinontech Co. Ltd (Beijing, China). Edaravone and 2,3,5Triphenyte-trazoliumchloride (TTC) were purchased from Sigma-Aldrich Co. Ltd. (USA).

\section{Sample preparation}

The standard mixture is a methanol solution, which is composed of notoginsenoside R1 (0.3 mg/mL), ginsenoside Rg1 (1.2 mg/mL), Rg2 (0.2 mg/mL), Rb1 $(1.0 \mathrm{mg} / \mathrm{mL})$ and $\mathrm{Rd}(0.1 \mathrm{mg} / \mathrm{mL})$.

The TSPN solution was obtained by accurately weighing power and dissolving in methanol, and finally, a concentrated solution of $4 \mathrm{mg} / \mathrm{mL}$ is obtained.

\section{HPLC instrumentation and conditions}

Analytical high performance liquid chromatography (HPLC) was performed on a Shimadzu LC-20AT system equipped with an LC-20 AT solvent delivery unit, an SIL20A autosampler, an SPD-M20A ultraviolet detector and a LabSolution Workstation. Chromatographic separation was carried on an Inertsil ODS-3 C18 column (4.6 x 150 $\mathrm{mm}, 5 \mu \mathrm{m}$ ) with the mobile phase consisting of acetonitrile (A) and $\mathrm{H}_{2} \mathrm{O}(\mathrm{B})$ at a flow rate of $1.0 \mathrm{~mL} / \mathrm{min}$. A gradient elution program was used as follows: 0-45 min, 20\% A; 45-65 min, 20-34\% A; 65-85 min, 34\% A;85-86 min, 34-90\% A; 86-96 min, 90\% A; 96-97 $\mathrm{min}, 20 \% \mathrm{~A}$. The injection volume was $10 \mu \mathrm{L}$. Detection wavelength was set at $203 \mathrm{~nm}$ and the column temperature was at $25^{\circ} \mathrm{C}$.

\section{Primary culture of rat cortical neurons}

Cortical neurons were collected from E18 Sprague-Dawley rat embryos [24]. Briefly, cortical neurons were dissociated by incubation for 15 min at $37{ }^{\circ} \mathrm{C}$ in $5 \mathrm{~mL}$ Hank's balanced salt solution free of calcium and magnesium (Life Technologies, CA) with $0.05 \%$ trypsin. Then dissociated neurons were fed with Neurobasal medium (Life 
Technologies) supplemented with 2\% B27 (Life Technologies). Neuronal cells were seeded on 96-well plates ( $1 \times 10^{5}$ per well) for MTT assay and $35 \mathrm{~mm}$ dishes $\left(8 \times 10^{5}\right.$ per well) for Western blot analysis. The medium was replaced by half every 3 days and the experiment was carried out at the 7th day in vitro (DIV).

\section{OGD/R model and drug exposure}

As a pretreatment, before OGD, incubated cortical neurons with TSPN or Edaravone for $2 \mathrm{~h}$. Then, replaced culture medium with a medium containing the drugs DMEM without glucose (Life Technologies). Next, the neurons were placed in a Modular Incubator Chamber (MIC-101; Billups-Rothenberg Inc, San Diego, CA, USA) for $4 \mathrm{~h}$, which saturated with $95 \% \mathrm{~N}_{2}$ and $5 \% \mathrm{CO}_{2}$ and formed a hypoxia environmental condition. After that, the neurons returned to the normoxic environment in a culture incubator in medium with TSPN or Edaravone. Cell viability, cell apoptosis, and cell death were measured at $24 \mathrm{~h}$ after OGD, and several signaling proteins were investigated at various time points after OGD. In each experiment, OGD groups were compared with normoxic controls supplied with normal cultural media under standard conditions.

\section{Analysis of neuronal cell viability}

Cell viability was proved using the MTT assay. Briefly, after $4 \mathrm{~h}$ of adding MTT, formazan was formed and become purple when dissolved in DMSO. The optical density (OD) values were detected in a multimode detector (Beckman Coulter, S. Kraemer Boulevard Brea, CA, USA) at $595 \mathrm{~nm}$. At last, using the following equation to calculate cell viability: cell viability $(\%)=\mathrm{OD}_{\text {sample }} / \mathrm{OD}_{\text {control }} \times 100 \%$.

\section{Detection of apoptosis by flow cytometry}

The neuroprotective effect of TSPN on OGD/R-induced cell apoptosis was monitored using an apoptosis detection kit Annexin VFITC/PI (propidium iodide) (Biouniquer Tech, Nanjing, China) by the manufacturer's instructions [25]. Briefly, cortical neurons were collected by centrifugation, washed twice with cold phosphatebuffered saline (PBS), and resuspended in Annexin-binding buffer. Subsequently, Annexin V-FITC and PI were added and incubated for $15 \mathrm{~min}$ in the dark at room 
temperature. The rate of apoptosis was analyzed by flow cytometry.

\section{Live/ Dead cell assay}

Cortical neurons were plated at a density of $6 \times 10^{4} /$ coverslip. Cell death was examined using Live/ Dead Cell Assay Kit (Abcam, Cambridge, UK) according to the manufacturer's instructions. In short, at room temperature, washed the cells twice with PBS and incubated for 15 min with $1000 \mathrm{x}$ buffer diluted to $5 \mathrm{x}$ buffer containing PBS in the dark. The live cells and dead cells were stained separately and photographed by a Zeiss Axio Imager A2 microscope (Carl Zeiss AG, Jena, Germany).

\section{Morphological analysis of dendrites}

MAP2 as a dendrite marker was stained in neurons. The number of positivelystained cells was calculated from images obtained by a Zeiss 700 confocal microscope (Carl Zeiss AG). In 3 separate experiments, a field of 10 was selected for each coverslip. Total dendritic branch points and the lengths of dendritic were counted by ImageJ software (National Institutes of Health, Maryland).

\section{Experimental animals}

A total of 138 male Wistar rats with 260-280 g were provided by Jinan Pengyue Experimental Animal Breeding Co. Ltd. (license: SCXK 20140007). Before the MCAO experiment, rats were adapted to 7 days with $12 \mathrm{~h}: 12 \mathrm{~h}$ equivalent light-dark cycles. The experimental procedures were approved by the Ethics Committee for Experimental Animal Center of Jinan University.

Randomly divided rats into 6 groups: Sham group (saline, $n=27$ ), MCAO group ( $\mathrm{MCAO}+$ saline, $\mathrm{n}=27)$, Edaravone group (MCAO + Edaravone, $\mathrm{n}=19$, Edaravone $6 \mathrm{mg} / \mathrm{kg}$ ), TSPN-high group (MCAO + TSPN, $\mathrm{n}=27$, TSPN $50 \mathrm{mg} / \mathrm{kg}$ ), TSPN-middle group (MCAO + TSPN, n = 19, TSPN 25 mg/kg), TSPN-low group (MCAO + TSPN, $\mathrm{n}=19$, TSPN $12.5 \mathrm{mg} / \mathrm{kg}$ ). Dosages were determined according to the clinical dose of TSPN. Rats in each group were intravenously (iv) administered appropriate doses at the onset of reperfusion and twice daily for 3 days.

\section{Middle cerebral artery occlusion (MCAO) model}

The MCAO model was based on the previously reported literature [26]. Briefly, rats were anesthetized in an anesthesia machine equipped with isoflurane (RWD Life 
Science Co. Ltd, Shenzhen, China), meanwhile, maintained rectal temperature of rats at $37{ }^{\circ} \mathrm{C}$ using a temperature-controlled heating pad over the surgical procedure. The right common carotid artery (CCA), external carotid artery (ECA) and internal carotid artery (ICA) were revealed through a $2 \mathrm{~cm}$ vertical incision in midline. A monofilament nylon suture was inserted from ECA into the ICA until it blocked the origin of the middle cerebral artery (MCA). After $2 \mathrm{~h}$ of occlusion, the nylon filament was withdrawn to reperfusion. Animals that underwent simulated surgery were treated in the same way, except for MCA occlusion.

\section{Neurobehavioral assessment}

Neurological sores in rats were blindly assessed using the following 4-point scaling system at $24 \mathrm{~h}$ or $72 \mathrm{~h}$ after ischemia reperfusion [27]: $0=$ no neurological deficits, $1=$ contralateral shoulder rotation and forelimb adduction when tail lifting, 2 $=$ the ability of the forelimbs to resist the opposite thrust decreased when transversely pushing the shoulder to the opposite side, and $3=$ circling to the contralateral side.

Tail hang test was performed on rats in each group at $72 \mathrm{~h}$ after cerebral ischemia. Rats were lifted 5 to $10 \mathrm{~cm}$ above the table for $5 \mathrm{~s}$ and repeated 20 times each. The number of cases in which the rotation angle of the rat's head exceeded $90^{\circ}$ was counted.

\section{TTC staining}

Staining with 2,3,5-triphenyltetrazolium chloride (TTC) was used to assess the cerebral infarction. Before staining, TTC power was prepared in normal saline. Rats were sacrificed at $72 \mathrm{~h}$ after reperfusion, the brains were placed at -80 for $10-12 \mathrm{~min}$. With the thickness of $2 \mathrm{~mm}$ per slice, brains were cut into 6 slices in coronal direction and placed in $2 \%$ TTC solution. The slices were incubated at $37{ }^{\circ} \mathrm{C}$ for $30 \mathrm{~min}$. After staining, normal tissue from the brain slice is shown in red, and the tissue affected by the infarction is shown in white. Infarct volume was analyzed using Image-Pro Plus image analysis software.

The volume of infarction and edema were calculated with the following formulas:

$$
\begin{aligned}
& \mathrm{V}_{\mathrm{i}}(\%)=\left[\left(\mathrm{V}_{\mathrm{c}}-\mathrm{V}_{\mathrm{ipn}}\right) / \mathrm{V}_{\mathrm{c}}\right] \times 100 \% \\
& \mathrm{~V}_{\mathrm{e}}(\%)=\left[\left(\mathrm{V}_{\mathrm{ip}}-\mathrm{V}_{\mathrm{c}}\right) / \mathrm{V}_{\mathrm{c}}\right] \times 100 \%
\end{aligned}
$$


$\mathrm{V}_{\mathrm{i}}$ represents infarct volume; $\mathrm{V}_{\mathrm{e}}$ represents edema volume; $\mathrm{V}_{\mathrm{c}}$ represents contralateral volume; $\mathrm{V}_{\mathrm{ipn}}$ represents ipsilateral non-infarct volume; $\mathrm{V}_{\mathrm{ip}}$ represents ipsilateral volume.

\section{Nissl Staining}

After sacrificing rats, they were perfused with saline and then with $4 \%$ paraformaldehyde (PFA) in phosphate buffer saline (PBS, 0.01 M, pH 7.4). Brains were carefully removed on ice, fixed for $24 \mathrm{~h}$ at $4{ }^{\circ} \mathrm{C}$ in $4 \%$ PFA. Brains were gradually dehydrated with ethanol and xylene and then immersed in liquid paraffin to embed brain tissue. Embed tissue was sliced into coronal sections $(4 \mu \mathrm{m})$. After that, slices were dewaxed, dehydrated, and dyed with Nissl staining solution (1\% cresyl violet). Then the slices were washed with ethanol and xylene as steps, and mounted with neutral resins and observed under a microscope (Olympus, Japan) at $400 \mathrm{x}$ magnification.

\section{Immunohistochemistry analysis}

The prepared paraffin slide $(4 \mu \mathrm{m})$ was subjected to antigen retrieval, thermally treated in a microwave, and washed within $0.01 \mathrm{M}$ PBS. The activity of endoperoxidase was blocked with $3 \% \mathrm{H}_{2} \mathrm{O}_{2}$ in methanol and nonspecific sites were blocked with a blocking solution containing 3\% BSA and 0.3\% Triton X-100 in PBS. And then the slides were incubated overnight at $4{ }^{\circ} \mathrm{C}$ with primary antibodies and incubated with biotinylated secondary antibodies for $1 \mathrm{~h}$. The sections were then stained with 3,30Diaminobenzidine tetrahydrochloride (DAB). The sections were finally coated with hematoxylin, dried in ethanol and xylene step by step before mounted. The slides were examined under microscope (Olympus, Japan) with $400 \mathrm{x}$ magnification.

\section{Immunofluorescence assay}

Rats were deeply anesthetized and perfused with saline and then with $4 \%$ PFA in PBS (0.01 M, pH 7.4). Brains were carefully removed on ice and fixed for $8 \mathrm{~h}$ at $4{ }^{\circ} \mathrm{C}$ in $4 \%$ PFA, then cryoprotected in sucrose $(10 \%, 20 \%$, and $30 \%$ sucrose in $0.01 \mathrm{M}$ PBS) at $4{ }^{\circ} \mathrm{C}$. Then they were embedded in opti-mum cutting temperature compound (O.C.T) (Sakura, Japan), and the embedded tissues were sliced into coronal sections (20 $\mu \mathrm{m})$ in a freezing microtome (Leica, Germany). Subsequently, slides were washed twice with 
0.01 M PBS for $10 \mathrm{~min}$, followed by incubation for $1 \mathrm{~h}$ in blocking solution containing $1 \%$ BSA and $0.3 \%$ Triton X-100 in PBS. After blocking, the slides were incubated overnight at $4{ }^{\circ} \mathrm{C}$ in the following mixed primary antibodies: LC3B (diluted 1:200, CST, USA) and NeuN (diluted 1:1000, CST, USA). The sections were then incubated for 1 h with the mixed secondary antibodies: Alexa Fluor 594 conjugated anti-rabbit IgG (diluted 1:200, CST, USA) and Alexa Fluor 488 conjugated anti-mouse IgG (diluted 1:200, CST, USA). Finally, the slides were mounted on 40,60-diamidino-2phenylindole (DAPI) for 20min and Antifade Mounting Medium (Beyotime, China). Staining patterns of the immunofluorescence were captured using an LSM800 laser scanning confocal microscope (ZEISS, Germany).

\section{Western blot analysis}

Cortical neurons or ischemic penumbras [3] were lysed in radio immunoprecipitation assay (RIPA) with protease inhibitors (Bimake, Houston, TX, USA) for $30 \mathrm{~min}$ at $4{ }^{\circ} \mathrm{C}$. And samples were centrifuged at $11,900 \mathrm{~g}$ at $4{ }^{\circ} \mathrm{C}$ for $15 \mathrm{~min}$ to obtain supernatants. Then the protein concentration was assessed using the bicinchoninic acid (BCA) kit (Pierce, USA). Total protein $(30 \mu \mathrm{g})$ was applied to each lane of $8 \%$ SDS-polyacrylamide gels and transferred to a poly-vinylidene fluoride (PVDF) membrane (Millipore, USA), incubated with 5\% albumin in bovine serum (BSA) blocking buffer. The PVDF membranes were incubated overnight at $4{ }^{\circ} \mathrm{C}$ with the following antibodies: phospho-Akt/Akt, phospho-mTOR (Ser2448)/mTOR, cleaved-Caspase3, LC3B, Beclin-1, $\beta$-actin or GAPDH antibodies (diluted 1:1000; CST, USA). The membranes were incubated with secondary antibody (diluted 1:2000, MultiSciences, China) for $1 \mathrm{~h}$ at room temperature. Subsequently, the membranes were incubated with ECL detection reagent (Millipore, USA), and the images were captured on an Amersham Imager 600 (GE, USA). The protein levels were analyzed by ImageJ.

\section{Statistical analysis}

All analyses were carried out using GraphPad Prism 5.0 (GraphPad Software Inc, USA). All data are presented as the mean values with the standard deviation (SD). Significant differences between more than two groups were evaluated using one-way ANOVA followed by Tukey's post hoc test for multiple comparisons when the data met 
the homogeneity of variance, as indicated in the figure legends. $P$ values less than 0.05 were considered significant.

\section{Results}

\section{Main Ingredients of TSPN}

According to HPLC analysis, the TSPN solution contains five components, such as notoginsenoside R1, ginsenoside $\mathrm{Rg} 1$, Re, Rb1, Rd (Figure 1). Under the experimental condition, the theoretical area for the absorption peak of the chromatogram of ginsenoside $\mathrm{Rg}_{1}$ was over 6,000, and chromatographic peak resolution of ginsenoside $\operatorname{Rg}_{1}$ and $\operatorname{Re}$ was $>1.5$.

\section{TSPN protect cortical neuronal against OGD/R-induced neuronal damage}

To explore the nontoxic dosage of TSPN for neurons, different concentrations of the TSPN $(400,100,50,25$ and $12.5 \mathrm{mg} / \mathrm{mL})$ were applied to cortical neurons for $24 \mathrm{~h}$, and cell viability was measured by MTT assay (Figure 2a). No evidence was found that TSPN had cytotoxicity at concentrations up to $50 \mathrm{mg} / \mathrm{mL}$ (Figure $2 \mathrm{a}$ ). To test whether TSPN protect cortical neurons from damage induced by OGD/R, cells were pretreated with clinically used Edaravone $(25 \mu \mathrm{M})$ as a positive control, or TSPN $(50,25,12.5$ and $1.25 \mathrm{mg} / \mathrm{mL}$ ) for $2 \mathrm{~h}$ before OGD. Edaravone and TSPN were also added to the whole OGD/R process. Both Edaravone and TSPN increased the cell viability of neurons affected by OGD/R when compared to the control group (Figure 2b), indicating that TSPN protect neurons from damage induced by OGD/R.

\section{TSPN reduce OGD/R-induced neuronal apoptosis}

To investigate whether the increase in cell viability by TSPN was due to a decrease in neuronal apoptosis, cell viability in $12.5 \mathrm{mg} / \mathrm{mL}$ TSPN or $25 \mu \mathrm{M}$ Edaravone was tested by flow cytometry with an Annexin V-FITC/PI Apoptosis Detection Kit (Figure 3a). According to the results, Edaravone or TSPN notably reduced the neuronal apoptosis (Figure 3a,c). Meanwhile, Live/Dead Cell Assay Kit was used to distinguish two kinds of cells by dyeing different fluorescent. Consistent with the result of flow cytometry, the percentage of dead cells was decreased by the treatment of Edaravone 
or TSPN (Figure 3b,d). These findings suggest that TSPN protect cortical neurons from apoptosis induced by OGD/R.

\section{TSPN protect morphological integrity of neurons after OGD/R}

To detect whether neuronal morphology is protected by TSPN after OGD/R, dendritic arborization was visualized by immunofluorescence staining using the dendrite marker MAP2 (Figure 4a). OGD/R induced remarkably atrophy of dendrites, which was evidenced by a reduction in the length and branches of the dendrite (Figure 4a-c). However, Edaravone and TSPN restored the dendrite morphology (Figure 4a-c). TSPN reverse the levels of Akt/mTOR and signaling proteins involved in autophagy and apoptosis in OGD/R-damaged neurons

We previously showed that Akt/mTOR signaling was inhibited during OGD [24]. Then, we investigated the effect of TSPN on Akt/mTOR signaling pathway. Consistently, the levels of phospho-Akt and phospho-mTOR were markedly decreased after OGD/R compared to the control group (Figure 5a-c), while the treatment with 12.5 $\mathrm{mg} / \mathrm{mL}$ TSPN increased the levels of phospho-Akt and phospho-mTOR (Figure 5a-c). Furthermore, OGD/R increased the levels of signaling proteins involved in autophagy and apoptosis, including LC3BII, Beclin-1, and cleaved-Caspase3. However, the expression of LC3BII and cleaved-Caspase 3 was reversed by TSPN $(12.5 \mathrm{mg} / \mathrm{mL})$ (Figure 5a,d-f).

TSPN alleviate neurological dysfunction, infarction, and brain edema caused by cerebral ischemia

No evidence was found in neurological scores for each group at $24 \mathrm{~h}$ after MCAO surgery, but the scores for each group decreased at $72 \mathrm{~h}$ (Figure 6a). To assess the neuroprotective effect of TSPN in rats, TSPN was administered by injection into the tail vein in 3 doses $(50 \mathrm{mg} / \mathrm{kg}, 25 \mathrm{mg} / \mathrm{kg} ; 12.5 \mathrm{mg} / \mathrm{kg})$ twice daily for 3 days after MCAO. High-dose TSPN and Edaravone improved neurological function in MCAO rats, because of their lower neurological scores (Figure 6a). Similarly, high-dose TSPN and Edaravone reduced the frequency of head rotation in MCAO rats in tail hang test (Figure 6b). Besides, the infarction area of the ischemic slice was measured by staining with TTC. There were more infarctions and edema than the similar hemisphere in the 
MCAO group. It is important to note that the infarction size of Edaravone group, highdose TSPN group and the medium-dose TSPN group was reduced compared to that of MCAO group (Figure 7a,b). The edema volume was also reduced by TSPN, but the difference was not statistically significant (Figure 7a,c).

\section{TSPN reduce neuronal damage in MCAO rats}

To observe the morphology of neurons, we used Nissl staining to differentiate neurons. Neurons in the hippocampus of the sham group were found to have a regular round soma, a distinct nucleus, visible Nissl bodies. However, in the MCAO group, neurons were arranged irregularly and disorderly, the nucleus was unclear and the number of Nissl bodies was low. Interestingly, Edaravone, high-dose TSPN and the medium-dose TSPN partially alleviated nerve damage in ischemic penumbra. These neurons mainly have clear nucleolus, increased Nissl bodies in the cytoplasm, but no obvious effect in the low-dose TSPN group (Figure 8).

\section{TSPN inhibit autophagy pathway in the ischemic penumbra of MCAO rats}

In cortical neurons after OGD/R, TSPN reversed the changes of Akt/mTOR pathway and autophagy pathway. However, whether TSPN have the same effect on MCAO rats is unknown. Firstly, by using Western blotting, we showed that TSPN restored the levels of p-mTOR (Figure 9a,b) and Beclin-1 (Figure 9a,c) in penumbra at $4 \mathrm{~h}$ following ischemia-reperfusion. Secondly, the expressions of Beclin-1 in ischemic penumbra and hippocampus CA1 area were examined using immunohistochemistry. Beclin-1 was up-regulated in ischemic penumbra of MCAO group when compared with Sham group (Figure 10a,b). Treatment of TSPN with high or middle doses or Edaravone may prevent the induction of Beclin-1 improvement by ischemia (Figure 10a,b). While all groups prevented the Beclin-1 in penumbra, there was no difference in the expression of Beclin-1 in the CA1 region (Figure 10a,c). Finally, the levels of LC3B and NeuN protein in ischemic penumbra were detected by immunofluorescence (Figure 11). As a result, LC3B protein increased in the MCAO group (Figure 11a,b), accelerating co-location with NeuN, which indicated autophagy pathway in rats was activated. Consistently, in the TSPN-treated group, LC3B reduced(Figure 11a,b), suggesting that TSPN interfere with autophagy on penumbra neurons and play a 
protective role in cerebral ischemia.

\section{Discussion}

In this study, on the one hand, evaluated the neuroprotective effect by using an oxygen-glucose deprivation/reoxygenation (OGD/R) model on cortex neurons, which mimics neuronal damage in ischemic stroke in vitro. On the other hand, the effect of TSPN in vivo was studied by MCAO rat model. The neuroprotective effect of TSPN in vivo was evaluated at the concentrations of 50,25 and $12.5 \mathrm{mg} / \mathrm{kg}$, high-dose TSPN ameliorated cerebral ischemia and neurological scores of MCAO rats. This article showed that activating the Akt/mTOR pathway and inhibiting the autophagy pathway were ways for TSPN to exert its protective effect.

OGD/R has been widely used in the brain ischemic injury model of in vitro. In this study, OGD/R was used to study the protective effect of TSPN. TSPN protected the cortical neurons from apoptosis and maintained the integrity of dendrites. It was reported that damaged neurons lose their communication ability with other neurons due to the loss of secondary or higher dendrites, leading to neurological dysfunction and cell death [28]. It is proposed that the change of dendritic alteration caused damage immediately, but under some conditions, it was a phenomenon of reversible effect [29]. Our findings suggest that TSPN protected dendrites from damage, which may be important for neuronal survival after OGD/R.

The injury to motor cortex and pyramidal tracts in MCA area can cause motor dysfunction, irreversible loss of synaptic activity in cortex and blockage of nerve transmission in the lower cortex [30]. The behavioral evaluation of MCAO rats, including neurological scores and tail hang test, showed obvious neurological and motor disorders in rats. TSPN significantly reduced these neurological deficits at 50 $\mathrm{mg} / \mathrm{kg}$. According to TTC staining, TSPN significantly reduced the infarct volume of MCAO rats, especially at $50 \mathrm{mg} / \mathrm{kg}$. Nissl staining also showed that TSPN reduced neuronal damage caused by cerebral ischemia in a dose-dependent manner. These findings demonstrate that TSPN have a benefit on cerebral ischemia whether in vitro or in vivo. 
TSPN are extracted from the Chinese herbal medicine Panax notoginseng, which are a typical multi-component traditional Chinese medicine ingredient, containing notoginsenoside $\mathrm{R} 1$, ginsenoside $\mathrm{Rg} 1, \mathrm{Rb} 1, \mathrm{Rd}, \mathrm{Re}$, and other saponins components, of which notoginsenoside R1, ginsenoside $\mathrm{Rg} 1$, Rb1 account for about $60 \%$ of TSPN content. Of course, TSPN have so many pharmacological activities that it may be related to its various chemical components. TSPN have been proved to have obvious advantages in anti-hypoxia, anti-aging, and improving human immunity [31-34]. Otherwise, TSPN have been proved to have a protective effect on nerve cells $[35,36]$.

In order to explore the neuroprotection mechanism of TSPN, the following signaling pathways were studied. First, both in the process of neuronal survival and axon growth, PI3k/Akt/mTOR signaling pathway is involved and is a crucial part. [37, 38]. Akt is the main downstream target of the PI3K signal transduction pathway, which promotes cell survival, prevents apoptosis, and maintains cells function. mTOR, which is one of the important parts of Akt, also is a necessary component in cell survival and differentiation [39, 40]. Beclin-1 is an essential molecule for the formation of autophagosome, which mediates the formation, maturation, and localization of autophagy-related proteins in phagocytic vesicles. Beclin-1 expression represents the degree of activation of autophagy pathway to a certain extent [11, 41]. Moreover, mTOR/ULK1 signaling pathway is one of the classical pathways regulating autophagy. Inhibition of mTOR signaling pathway will induce the formation of ULK1 complex, thus promoting the activation of Beclin-1 and the formation of autophagosome. We found that TSPN inhibited the expression of Beclin-1 in both ischemic penumbra of rats after MCAO and cultured cortical neurons after OGD/R. The phosphorylation of mTOR was decreased in both models, but was reversed after treatment of TSPN. These results suggest that TSPN inhibit autophagy by upregulating mTOR whereas downregulating Beclin-1 expression, which may be the mechanism of the neuroprotection of TSPN.

\section{Conclusions}

This study explores the neuroprotective mechanism of TSPN in both in vitro 
OGD/R-injury neurons and in vivo MCAO rats. We found that TSPN promote neuronal survival and protect dendrite integrity after $\mathrm{OGD} / \mathrm{R}$, and have potential therapeutic effect on alleviating neurological deficits and reversing neuronal loss. TSPN inhibit autophagy induced by ischemic damage by promoting mTOR-and inhibiting Beclin-1dependent signaling pathways, which may be the mechanism that underlies the neuroprotective activity of TSPN.

\section{Abbreviations}

TSPN, total saponins of Panax notoginseng; OGD/R, Oxygen-glucose deprivation/reoxygenation; MTT, 3-(4, 5-dimethylthiazol-2-yl)-2,5-diphenyl tetrazolium bromide; MCAO, Rat middle cerebral artery occlusion; DMSO, dimethyl sulfoxide; TTC, 2,3,5-Triphenyte-trazoliumchloride; OD, optical density; PBS, phosphate-buffered saline; CCA, common carotid artery; ECA, external carotid artery; ICA, internal carotid artery; MCA, middle cerebral artery; DAB, 3,30Diaminobenzidine tetrahydrochloride; BCA, bicinchoninic acid; PVDF, polyvinylidene fluoride; BSA, bovine serum.

\section{Ethics approval and consent to participate}

Not applicable

\section{Consent for publication}

Not applicable

\section{Availability of data and materials}

Not applicable

\section{Competing interests}

All authors report no conflicts of interest and no competing financial interests exist.

\section{Author's contributions}


Man-Mei Li, Wen-Cai Ye and Lei Shi conceived and designed the study, Hua-Feng Liang, Dong-Ping Wu and Li-Li Zhang performed the in vivo experiments and collected the data; Yu-Wei Pan and Gen-Yun Tang performed the in vitro experiments and collected the data; Chun-Lin Fan, Ming-Chen and Guan-Ping Liu provided and tested the compound. Dong-Ping Wu, Yu-Wei Pan, Hua-Feng Liang, Man-Mei Li and Lei Shi wrote the manuscript; Man-Mei Li and Lei Shi revised the work for intellectual content and context; All the authors read and approved the manuscript.

\section{Acknowledgements}

This research was supported by National Key R\&D Program of China (2017YFC1703800), Science and Technology Program of Guangzhou (202002030294), Science and Technology Key Project of Guangdong Province, China (2020B1111110004), Local Innovative and Research Teams Project of Guangdong Pearl River Talents Program (2017BT01Y036) and Key Realm R\&D Program of Guangdong province (2019B030335001). The authors would like to thank the key laboratory for providing necessary facilities to ensure the research completed. 


\section{Reference}

1. Pandian JD, Gall SL, Kate MP, Silva GS, Akinyemi RO, Ovbiagele BI, et al. Prevention of stroke: a global perspective. The Lancet. 2018;392:1269-78.

2. Mueller M, Khamis D, Srivastava D, Exadaktylos AK, Pfortmueller CA. Understanding Refugees' Health. Seminars In Neurology. 2018;38:152-62.

3. Nagasawa H, Kogure K. Correlation between cerebral blood flow and histologic changes in a new rat model of middle cerebral artery occlusion. Stroke. 1989;20:1037-43.

4. Kim J, Fann DY, Seet RCS, Jo D, Mattson MP, Arumugam TV. Phytochemicals in Ischemic Stroke. NeuroMolecular Medicine. 2016;18:283-305.

5. Astrup J, Siesjo BK, Symon L. Thresholds in cerebral ischemia the ischemic penumbra. Stroke. 1981;12:723-5.

6. Chauhan A, Sharma U, Jagannathan NR, Reeta KH, Gupta YK. Rapamycin protects against middle cerebral artery occlusion induced focal cerebral ischemia in rats. Behavioural Brain Research. 2011;225:603-9.

7. Wen Y, Sheng R, Zhang L, Han R, Zhang X, Zhang X, et al. Neuronal injury in rat model of permanent focal cerebral ischemia is associated with activation of autophagic and lysosomal pathways. Autophagy. 2008;4:762-9.

8. Zhan L, Liu L, Li K, Wu B, Liu D, Liang D, et al. Neuroprotection of hypoxic postconditioning against global cerebral ischemia through influencing posttranslational regulations of heat shock protein 27 in adult rats. Brain Pathology. 2017;27:822-38.

9. Li X, Li J, Qian J, Zhang D, Shen H, Li X, et al. Loss of Ribosomal RACK1 (Receptor for Activated Protein Kinase C 1) Induced by Phosphorylation at T50 Alleviates Cerebral Ischemia-Reperfusion Injury in Rats. Stroke. 2019;50:16271.

10. Li Y, Su F, Wang L, Liu B, Shi Z, Deng J. Autophagy and hypoxic ischemic brain injuries. Acta Physiologica Sinica. 2017;69:316-24.

11. Brady NR, HamacherBrady A, Yuan H, Gottlieb RA. The autophagic response to nutrient deprivation in the hl-1 cardiac myocyte is modulated by Bcl-2 and 
sarco/endoplasmic reticulum calcium stores. Febs Journal. 2007;274:3184-97.

12. Wang F, Wang S, Chai L, Zhang Y, Guo H, Hu L. Xueshuantong injection (lyophilized) combined with salvianolate lyophilized injection protects against focal cerebral ischemia/reperfusion injury in rats through attenuation of oxidative stress. Acta Pharmacologica Sinica. 2018;39:998-1011.

13. Sheng S, Wang J, Wang L, Liu H, Li P, Liu M, et al. Network pharmacology analyses of the antithrombotic pharmacological mechanism of Fufang Xueshuantong Capsule with experimental support using disseminated intravascular coagulation rats. Journal Of Ethnopharmacology. 2014;154:73544.

14. Han S, Chen Y, Wang J, Zhang Q, Han B, Ge Y, et al. Anti-thrombosis Effects and Mechanisms by Xueshuantong Capsule Under Different Flow Conditions. Frontiers In Pharmacology. 2019;10.

15. Huang Y, Guo B, Shi B, Gao Q, Zhou Q. Chinese Herbal Medicine Xueshuantong Enhances Cerebral Blood Flow and Improves Neural Functions in Alzheimer's Disease Mice. Journal Of Alzheimers Disease. 2018;63:1089107.

16. Gao L, Zhao H, Liu Q, Song J, Xu C, Liu P, et al. Improvement of hematoma absorption and neurological function in patients with acute intracerebral hemorrhage treated with Xueshuantong. Journal Of the Neurological Sciences. 2012;323:236-40.

17. Liu L, Zhu L, Zou Y, Liu W, Zhang X, Wei X, et al. Panax notoginseng Saponins Promotes Stroke Recovery by Influencing Expression of Nogo-A, NgR and p75NGF, in Vitro and in Vivo. Biological and Pharmaceutical Bulletin. $2014 ; 37: 560-8$

18. Shi X, Yu W, Yang T, Liu W, Zhao Y, Sun Y, et al. Panax notoginseng saponins provide neuroprotection by regulating NgR1/RhoA/ROCK2 pathway expression, in vitro and in vivo. Journal of Ethnopharmacology. 2016;190:30112.

19. Zhang J, Zhang B, Du M, Wang X, Li W. Chinese preparation Xuesaitong 
promotes the mobilization of bone marrow mesenchymal stem cells in rats with cerebral infarction. Neural Regeneration Research. 2016;11:292-7.

20. Shen Q, Li J, Zhang C, Wang P, Mohammed A, Ni S, et al. Panax notoginseng saponins reduce high-risk factors for thrombosis through peroxisome proliferator-activated receptor $-\gamma$ pathway. Biomedicine \& Pharmacotherapy. 2017;96:1163-9.

21. Shi X, Yu W, Liu L, Liu W, Zhang X, Yang T, et al. Panax notoginseng saponins administration modulates pro- /anti-inflammatory factor expression and improves neurologic outcome following permanent MCAO in rats. Metabolic Brain Disease. 2017;32:221-33.

22. Huang X, Tan H, Chen B, Deng C. Combination of Total Astragalus Extract and Total Panax Notoginseng Saponins Strengthened the Protective Effects on Brain Damage through Improving Energy Metabolism and Inhibiting Apoptosis after Cerebral Ischemia-Reperfusion in Mice. Chinese Journal of Integrative Medicine. 2017;23:445-52.

23. Zhou J, Yang Y, Huang Z, Zou X, Liang X. Effect of Panax notoginseng Saponins on Cisplatin-Induced Kidney Injury by Enhancing Mitochondrial Autophagy Via the HIF-1 $\alpha$ /BNIP3 Pathway. Chin Pharm J. 2017;52:196-200.

24. Shi Q, Zhang Q, Peng Y, Ying X, Lei W. A natural diarylheptanoid protects cortical neurons against oxygen-glucose deprivation-induced autophagy and apoptosis. Journal of Pharmacy \& Pharmacology. 2019;71:1110-8.

25. Xiao HL, Zhang QH, Peng YH, Tang GY, Liao YM, Zhuang XJ, et al. 7-(4Hydroxy-3-methoxypheny1)-1-pheny1-4E-hepten-3-one alleviates A beta 1-42 induced cytotoxicity through PI3K-mTOR pathways. Biochemical And Biophysical Research Communications. 2017;484:365-71.

26. Longa E, Weinstein P, Carlson S, Cummins R. Reversible middle cerebral artery occlusion without craniectomy in rats. Stroke. 1989;20:84-91.

27. Bederson J, Pitts L, Tsuji M, Nishimura M, Davis R, Bartkowski H. Rat middle cerebral artery occlusion: evaluation of the model and development of a neurologic examination. Stroke. 1986;17:472-6. 
28. Furutani R, Kibayashi K. Morphological Alteration and Reduction of MAP2Immunoreactivity in Pyramidal Neurons of Cerebral Cortex in a Rat Model of Focal Cortical Compression. Journal of Neurotrauma. 2012;29:1266-76.

29. Hendrickson ML, Ling C, Kalil RE. Degeneration of Axotomized Projection Neurons in the Rat dLGN: Temporal Progression of Events and Their Mitigation by a Single Administration of FGF2. Plos One. 2012;7:e46918.

30. Bolay H, Dalkara T. Mechanisms of motor dysfunction after transient MCA occlusion: persistent transmission failure in cortical synapses is a major determinant. Stroke. 1998;29:1988-94.

31. XiYang Y, Wang Y, Zhao Y, Ru J, Lu B, Zhang Y, et al. Sodium Channel VoltageGated Beta 2 Plays a Vital Role in Brain Aging Associated with Synaptic Plasticity and Expression of COX5A and FGF-2. Molecular Neurobiology. 2016;53(2):955-67.

32. Li J, Chen Y, Zhang L, Xing L, Xu H, Wang Y, et al. Total saponins of panaxnotoginseng promotes lymphangiogenesis by activation VEGF-C expression of lymphatic endothelial cells. Journal Of Ethnopharmacology. 2016;193:293-302.

33. Li H, Deng C, Chen B, Zhang S, Liang Y, Luo X. Total saponins of Panax Notoginseng modulate the expression of caspases and attenuate apoptosis in rats following focal cerebral ischemia-reperfusion. Journal Of Ethnopharmacology. 2009;121(3):412-8.

34. Zhang Y, Zhang H, Zhang G, Fan J, Li X, Liu Y, et al. Panax notoginseng saponins attenuate atherosclerosis in rats by regulating the blood lipid profile and an anti-inflammatory action. Clinical And Experimental Pharmacology And Physiology. 2008;35(10):1238-44.

35. Zhou L, Huang PP, Chen LL, Wang P. Panax Notoginseng Saponins Ameliorate A $\beta$-Mediated Neurotoxicity in C. elegans through Antioxidant Activities. Evid Based Complement Alternat Med. 2019;2019:7621043.

36. Xiang H, Liu Y, Zhang B, Huang J, Li Y, Yang B, et al. The antidepressant effects and mechanism of action of total saponins from the caudexes and leaves 
of Panax notoginseng in animal models of depression. Phytomedicine. 2011;18(8-9):731-8.

37. Read DE, Gorman AM. Involvement of Akt in neurite outgrowth. Cellular and Molecular Life Sciences. 2009;66:2975-84.

38. Sun Z, Yang H, Wang Z, Pan J, Hong Z, Chen S. Erythropoietin prevents PC12 cells from beta-amyloid-induced apoptosis via PI3K/Akt pathway. Translational Neurodegeneration. 2012;1:7.

39. Ouyang Y. Survival- and Death-Promoting Events after Transient Cerebral Ischemia: Phosphorylation of Akt, Release of Cytochrome C, and Activation of Caspase-Like Proteases. Journal of Cerebral Blood Flow \& Metabolism. 1999;19:1126-35.

40. Castaneda CA, Funes HC, Gomez HL, Ciruelos EM. The phosphatidyl inositol 3-kinase/AKT signaling pathway in breast cancer. Cancer and Metastasis Reviews. 2010;29:751-9.

41. He C, Klionsky DJ. Regulation Mechanisms and Signaling Pathways of Autophagy. Annual Review Of Genetics. 2009;43:67-93. 


\section{Figure Captions}

Figure 1. HPLC chromatogram. (a). The mixture of standards. (b). The TSPN solutions. 1. notoginsenoside R1, 2. ginsenoside $\operatorname{Rg} 1$, 3. ginsenoside $\mathrm{Re}$, 4. ginsenoside $\mathrm{Rb} 1,5$. ginsenoside $\mathrm{Rd}$.

Figure 2. TSPN protect cortical neurons against OGD/R-induced cell injury. (a) Cortical neurons were treated with different concentrations of TSPN and cell viabilities were determined after $24 \mathrm{~h}$. $* P<0.05$, $* * P<0.01$ vs control, $\mathrm{n}=3$. (b) Different concentrations of TSPN or Edaravone were added in cortical neurons $2 \mathrm{~h}$ before OGD, as well as the whole process of OGD/R. Cell viability was detected by MTT assay. ${ }^{\# \# \# P} P<0.001$ vs Control. $* P<0.05, * * P<0.01$ vs OGD/R group, $\mathrm{n}=3$. The data are expressed as means $\pm \mathrm{SD}$.

Figure 3. TSPN reduce OGD/R-induced neuronal apoptosis. (a) Apoptotic cells were detected using Annexin V/PI staining by flow cytometry. (b) The live or dead cells were determined using the Live/Dead cell assay kit $(10 \times$ objective, scale bar $=100 \mu \mathrm{m})$. (c) Cell apoptosis is presented as a combined percentage of Annexin $\mathrm{V}^{+} / \mathrm{PI}^{-}$and Annexin $\mathrm{V}^{+} / \mathrm{PI}^{+}$cells. ${ }^{\# \#} P<0.001$ vs Control. ${ }^{*} P<0.05$, $* * * P<0.001$ vs DMSO group. (d) The percentage of dead cells was counted. ${ }^{\# \#} P<0.01$ vs Control. $* * P<0.01$ vs DMSO group, $\mathrm{n}=3$. The data are expressed as means $\pm \mathrm{SD}$.

Figure 4. TSPN reverse OGD/R-induced dendritic abnormalities. (a) Dendrites were shown by MAP2 staining (40x objective, scale bar $=20 \mu \mathrm{m})$. (b) Total dendrite branches were counted. (c) Total dendrite lengths were measured. ${ }^{\# \#} P<0.01$ vs Control, $n=3$. The data are expressed as means \pm SD.

Figure 5. TSPN restore Akt/mTOR pathway and inhibits the OGD/R-induced pathways involved in autophagy and apoptosis. (a) Levels of p-AKT, p-mTOR, LC3B, Beclin-1, and cleaved-Caspase 3 in OGD/R-damaged neurons are reversed by TSPN. (b-f) Relative band density of p-AKT (b), p-mTOR (c), Beclin-1 (d), cleaved-Caspase3 (e), and LC3BII (f) were measured using ImageJ. ${ }^{\#} P<0.05$ vs control, $* \mathrm{P}<0.05, * * \mathrm{P}<0.01$ vs OGD/R group, $\mathrm{n}=3$, The data are expressed as means $\pm \mathrm{SD}$. Figure 6. TSPN improve the rats motor function at $72 \mathrm{~h}$ after reperfusion. (a) Neurological score of rats at 24 and $72 \mathrm{~h}$ after ischemia-reperfusion. ${ }^{\# \#} P<0.001$ vs 
Sham group, $* P<0.05$ vs MCAO group, $\mathrm{n}=6$. Analyzed using two-way ANOVA. (b) Head rotation frequency at $72 \mathrm{~h}$ after cerebral ischemia-reperfusion. ${ }^{\# \#} P<0.001$ vs Sham group, $* P<0.05, * * * P<0.001$ vs MCAO group, $\mathrm{n}=6$. The data are expressed as means $\pm \mathrm{SD}$.

Figure 7. TSPN reduce infarction resulted from cerebral ischemia in MCAO rats. (a) TTC staining was used to detect cerebral infarction in rats of each group. (b) The infarct volume was determined by using Image-Pro Plus. (c) Statistical results of brain edema volume in each group. ${ }^{\# \# \#} P<0.001$ vs Sham group, $* P<0.05, * * P<0.01$ vs MCAO group, $\mathrm{n}=6$. The data are expressed as means $\pm \mathrm{SD}$.

Figure 8. Nissl staining of ischemic penumbra $(400 \times$, scale bar $=50 \mu \mathrm{m}, \mathrm{n}=7)$ There was a big difference in morphology between normal neurons and abnormal neurons. The former had normal morphology, clear nucleolus, abundant cytoplasm and intact structure. The latter had shrinkage and deep staining.

Figure 9. Modulation of the protein levels of mTOR, p-mTOR, and Beclin-1 in ischemic penumbra. (a) Representative bands of mTOR, p-mTOR, and Beclin-1 detected by Western blot analysis. (b-c) Quantification of expression levels of p-mTOR (b) and Beclin-1(c). $* P<0.05,{ }^{*} * P<0.001$ vs MCAO group, $\mathrm{n}=4$. The data are expressed as means $\pm \mathrm{SD}$.

Figure 10. Immunohistochemistry was used to detect the expression of Beclin-1 in ischemic penumbra and hippocampus CA1 area of MCAO rats. (a) The average optical densities of Beclin-1 in ischemic penumbra (b) and hippocampal CA1 region (c) were analyzed using ImageJ $(400 \times$, scale bar $=50 \mu \mathrm{m})$. ${ }^{\# \# \# ~} P<0.001$ vs Sham group, $* P<0.05$ vs MCAO group, $\mathrm{n}=6$. The data are expressed as means $\pm \mathrm{SD}$.

Figure 11. TSPN inhibit expression of LC3B in ischemic penumbra of MCAO rats. (a) Expressions of LC3B were identified by immunofluorescence. (b) The fluorescence intensity of LC3B was analyzed using ImageJ (40x objective, scale bar $=20 \mu \mathrm{m}$ ). ${ }^{\# \# \# P} P<0.001$ vs Sham group, ${ }^{* *} P<0.01$ vs MCAO group, $\mathrm{n}=4$. The data are expressed as means $\pm \mathrm{SD}$. 
Figures

$\mathbf{a}$

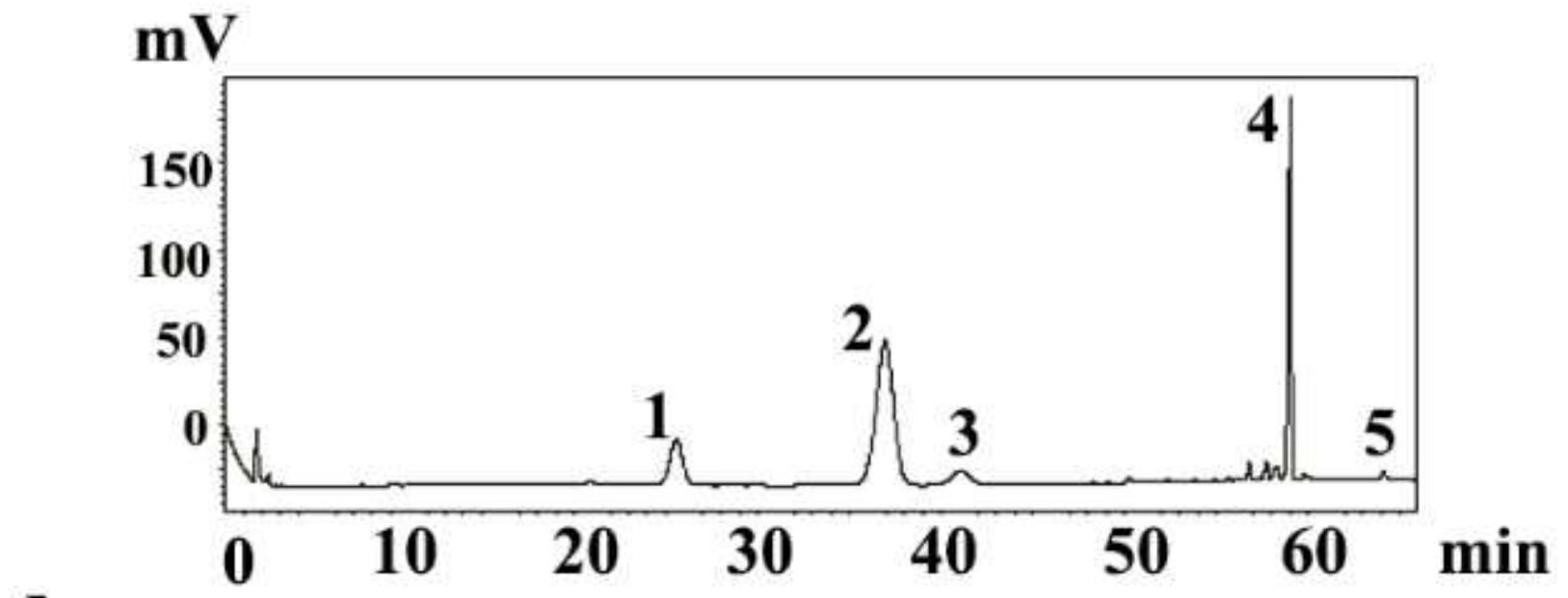

b

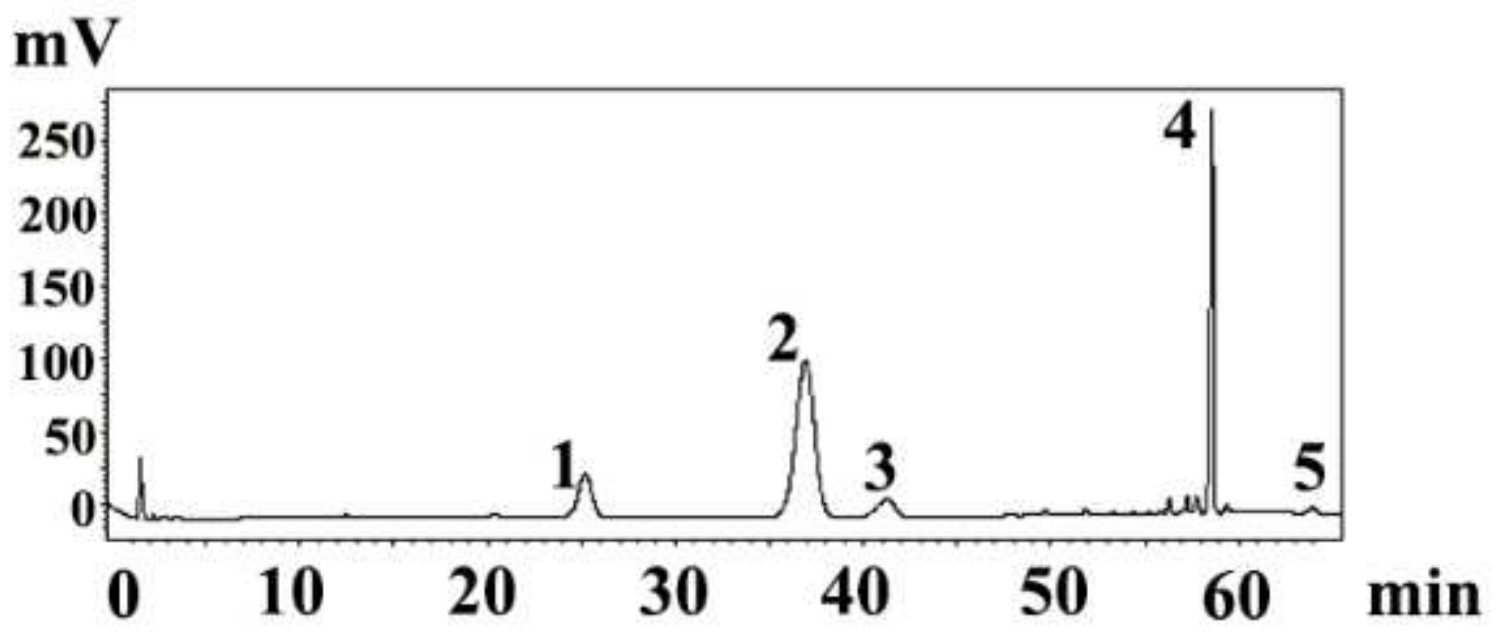

Figure 1

HPLC chromatogram. (a). The mixture of standards. (b). The TSPN solutions. 1. notoginsenoside R1, 2. ginsenoside Rg1, 3. ginsenoside Re, 4. ginsenoside Rb1, 5. ginsenoside Rd. 
$\mathbf{a}$

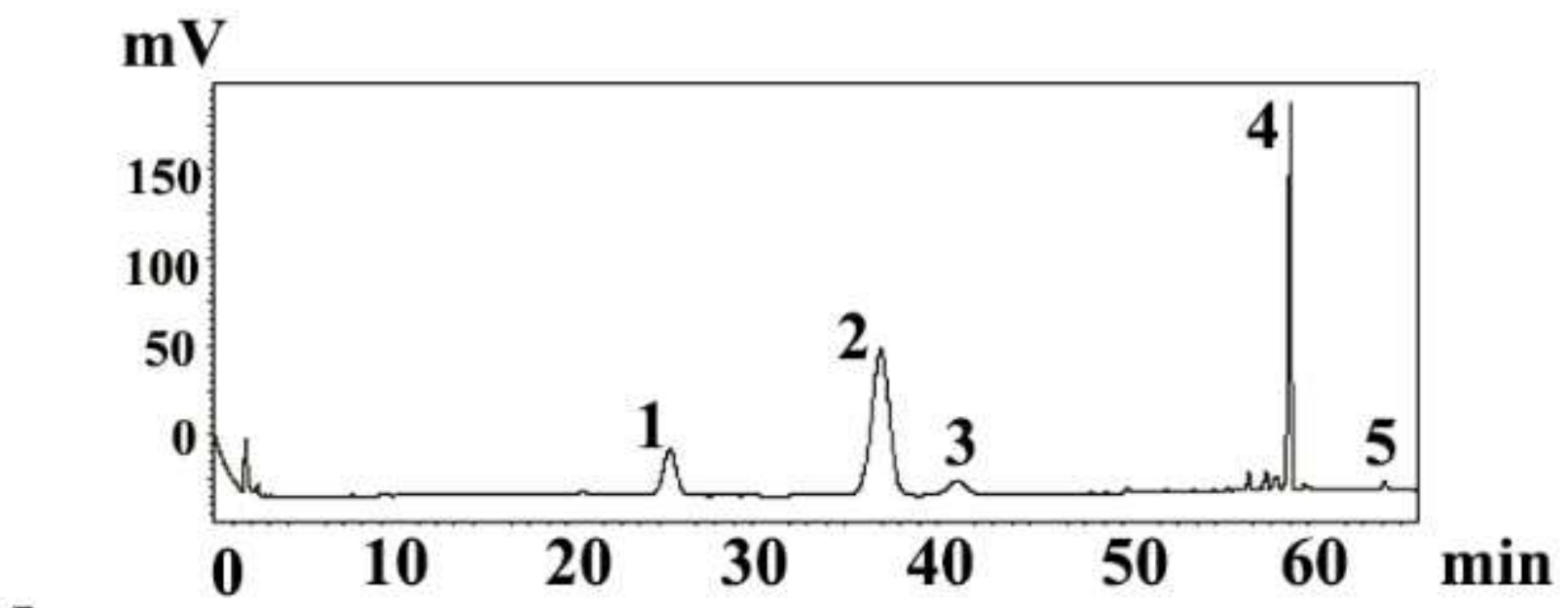

b

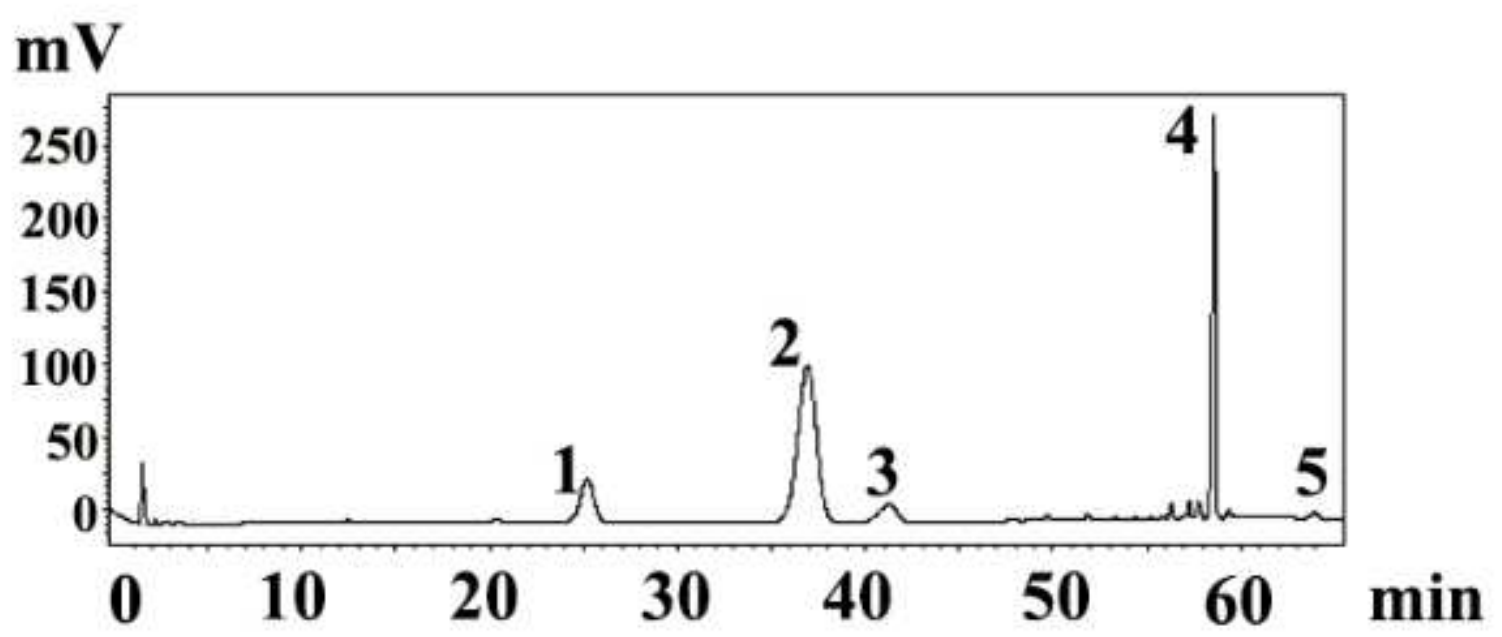

Figure 1

HPLC chromatogram. (a). The mixture of standards. (b). The TSPN solutions. 1. notoginsenoside R1, 2. ginsenoside Rg1, 3. ginsenoside Re, 4. ginsenoside Rb1, 5. ginsenoside Rd. 
$\mathbf{a}$

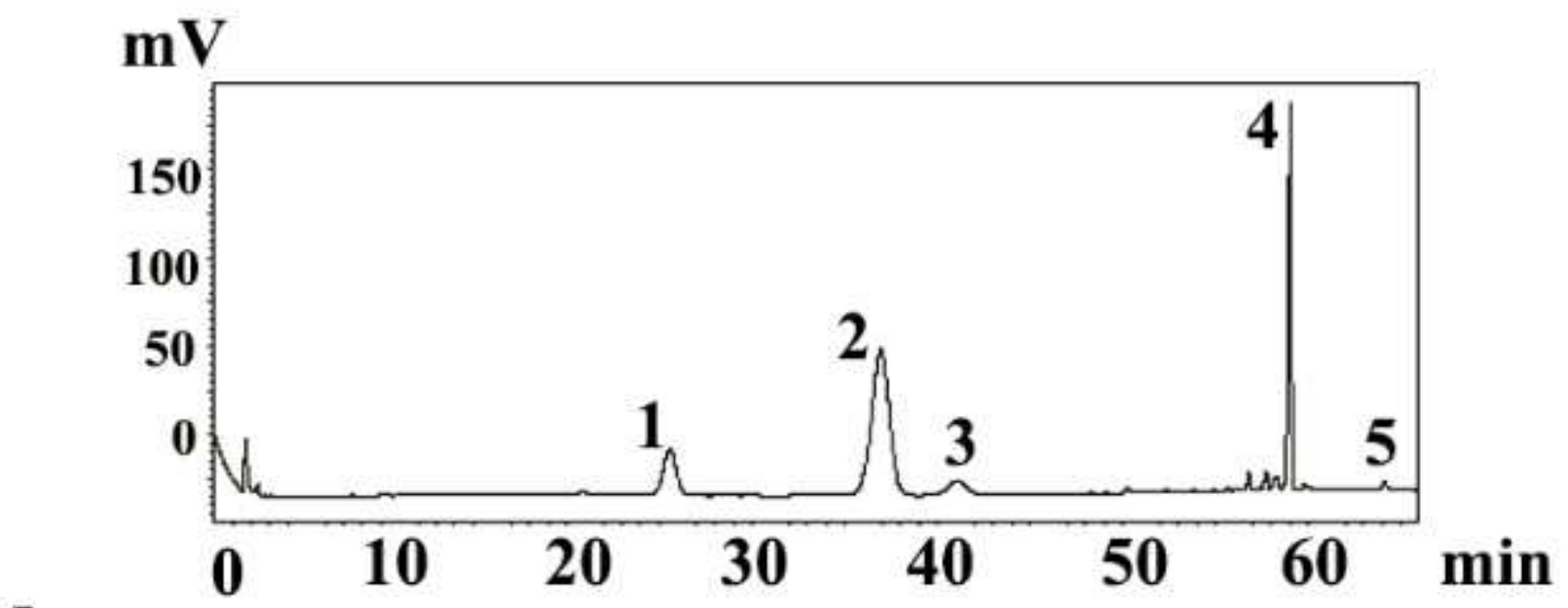

b

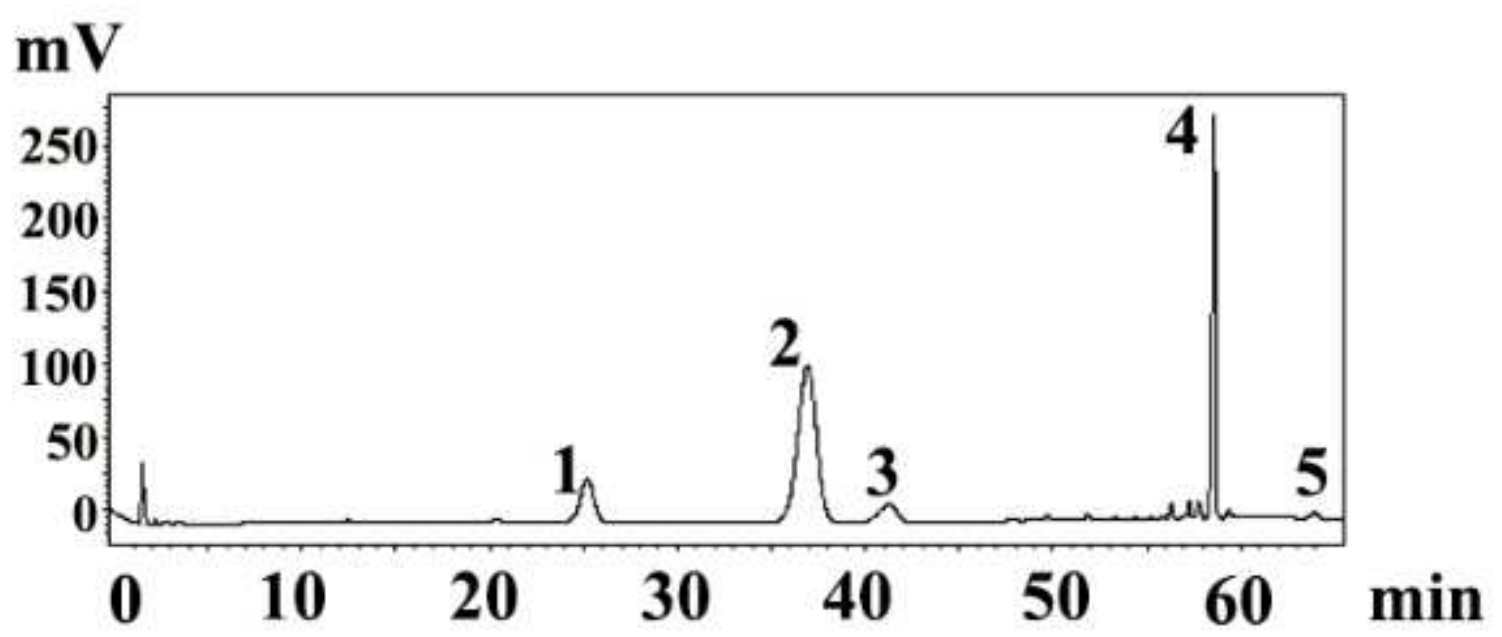

Figure 1

HPLC chromatogram. (a). The mixture of standards. (b). The TSPN solutions. 1. notoginsenoside R1, 2. ginsenoside Rg1, 3. ginsenoside Re, 4. ginsenoside Rb1, 5. ginsenoside Rd. 


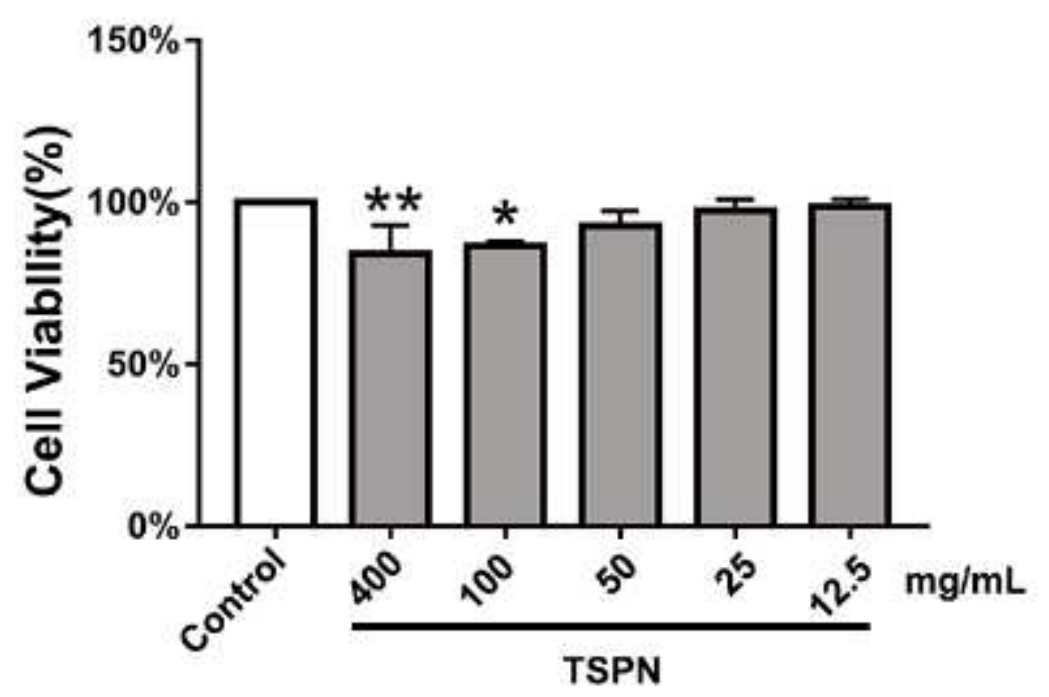

b

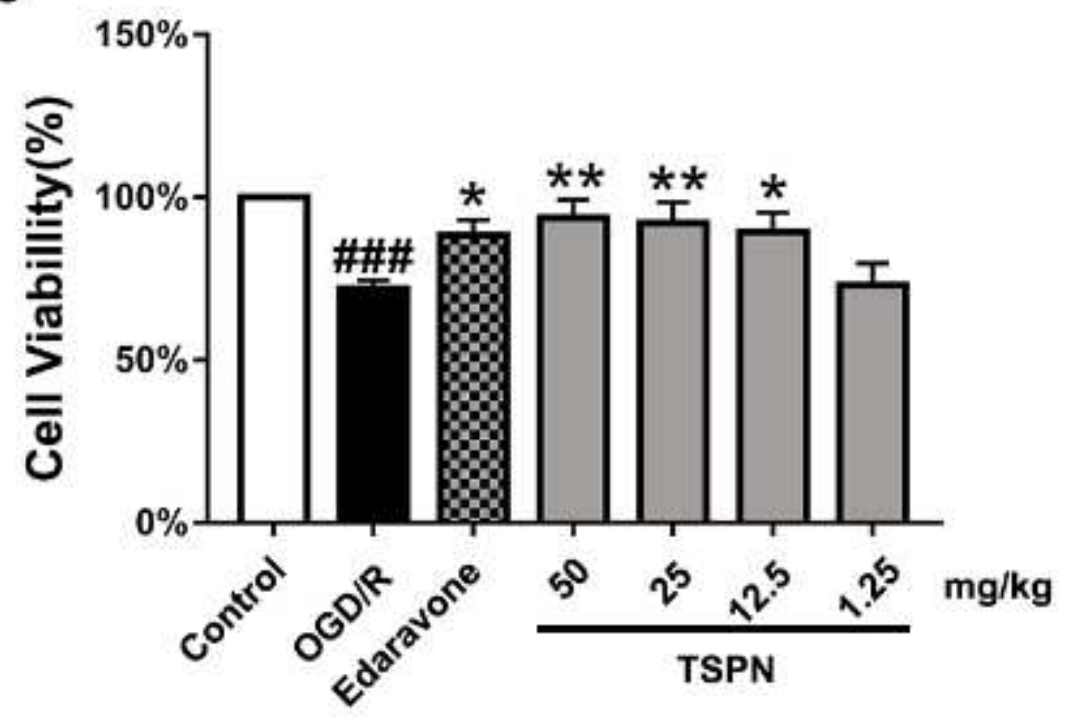

Figure 2

TSPN protect cortical neurons against OGD/R-induced cell injury. (a) Cortical neurons were treated with different concentrations of TSPN and cell viabilities were determined after $24 \mathrm{~h}$. ${ }^{*} \mathrm{P}<0.05,{ }^{*} \mathrm{P}<0.01$ vs control, $n=3$. (b) Different concentrations of TSPN or Edaravone were added in cortical neurons $2 \mathrm{~h}$ before OGD, as well as the whole process of OGD/R. Cell viability was detected by MTT assay. \#\#\#P< 0.001 vs Control. ${ }^{*} \mathrm{P}<0.05,{ }^{\star *} \mathrm{P}<0.01$ vs $\mathrm{OGD} / \mathrm{R}$ group, $\mathrm{n}=3$. The data are expressed as means $\pm \mathrm{SD}$. 


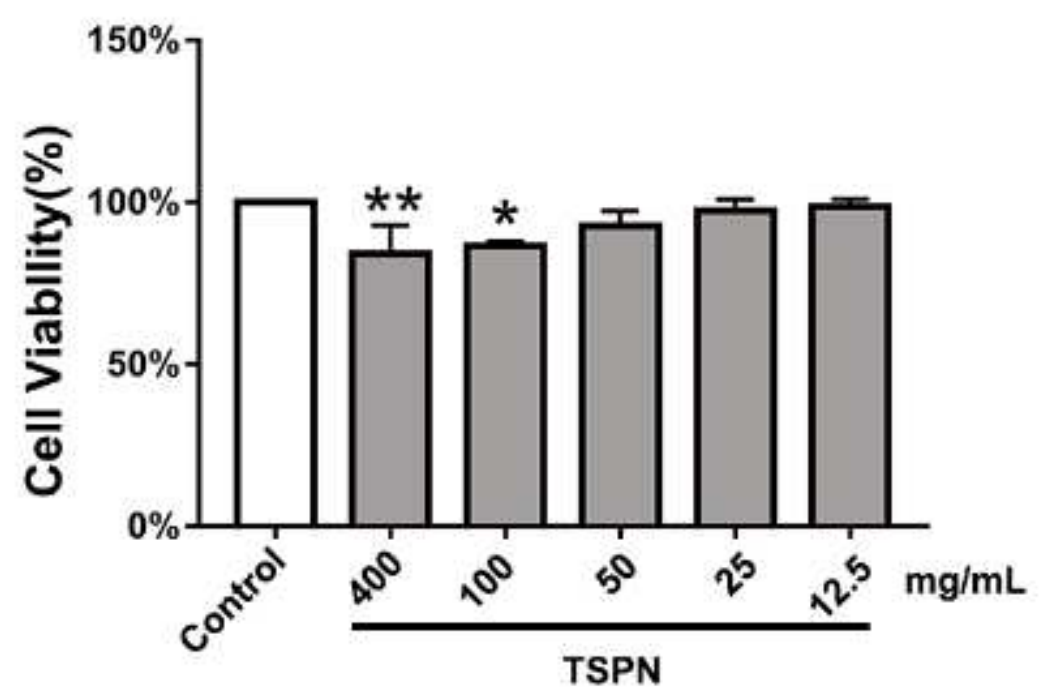

b

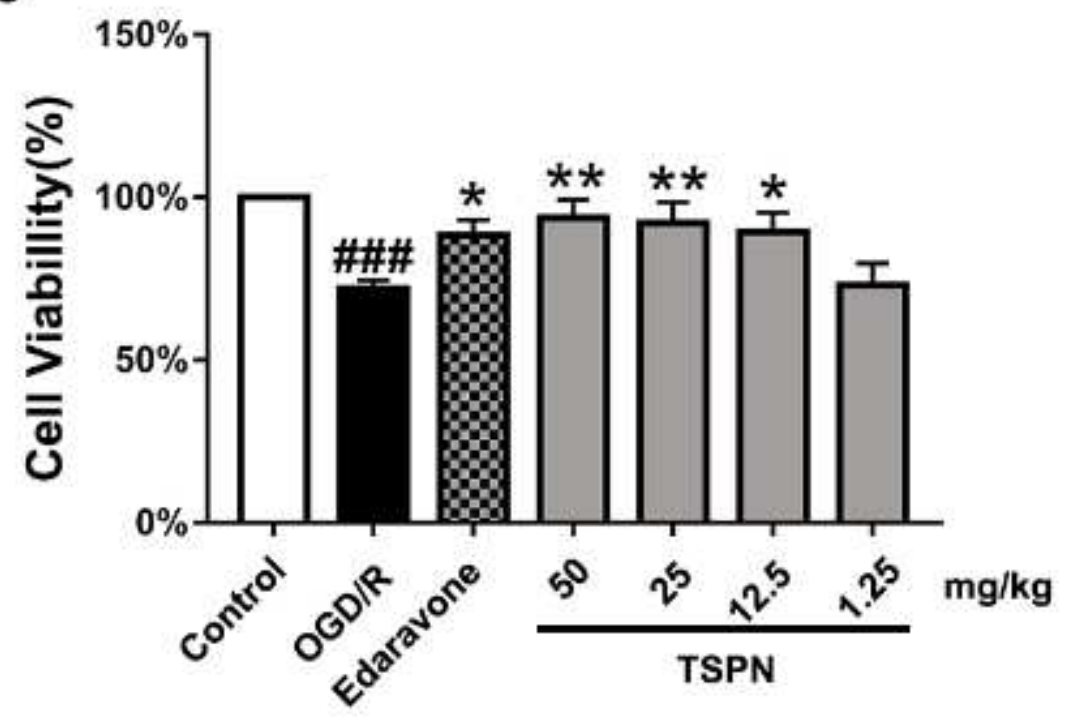

Figure 2

TSPN protect cortical neurons against OGD/R-induced cell injury. (a) Cortical neurons were treated with different concentrations of TSPN and cell viabilities were determined after $24 \mathrm{~h}$. ${ }^{*} \mathrm{P}<0.05,{ }^{*} \mathrm{P}<0.01$ vs control, $n=3$. (b) Different concentrations of TSPN or Edaravone were added in cortical neurons $2 \mathrm{~h}$ before OGD, as well as the whole process of OGD/R. Cell viability was detected by MTT assay. \#\#\#P< 0.001 vs Control. ${ }^{*} \mathrm{P}<0.05,{ }^{\star *} \mathrm{P}<0.01$ vs $\mathrm{OGD} / \mathrm{R}$ group, $\mathrm{n}=3$. The data are expressed as means $\pm \mathrm{SD}$. 


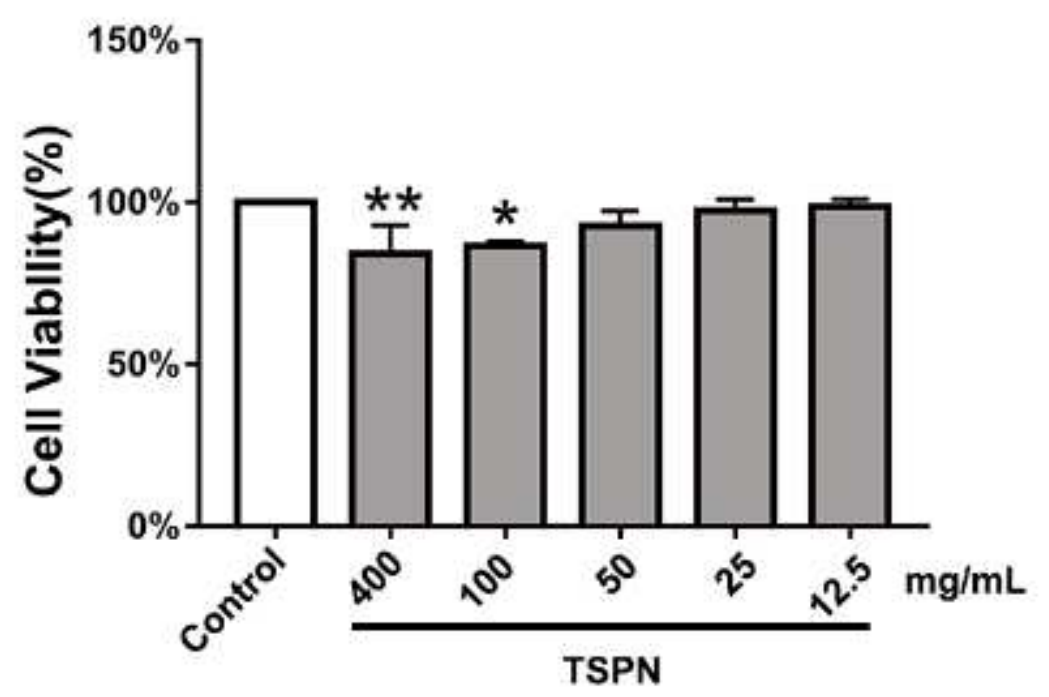

b

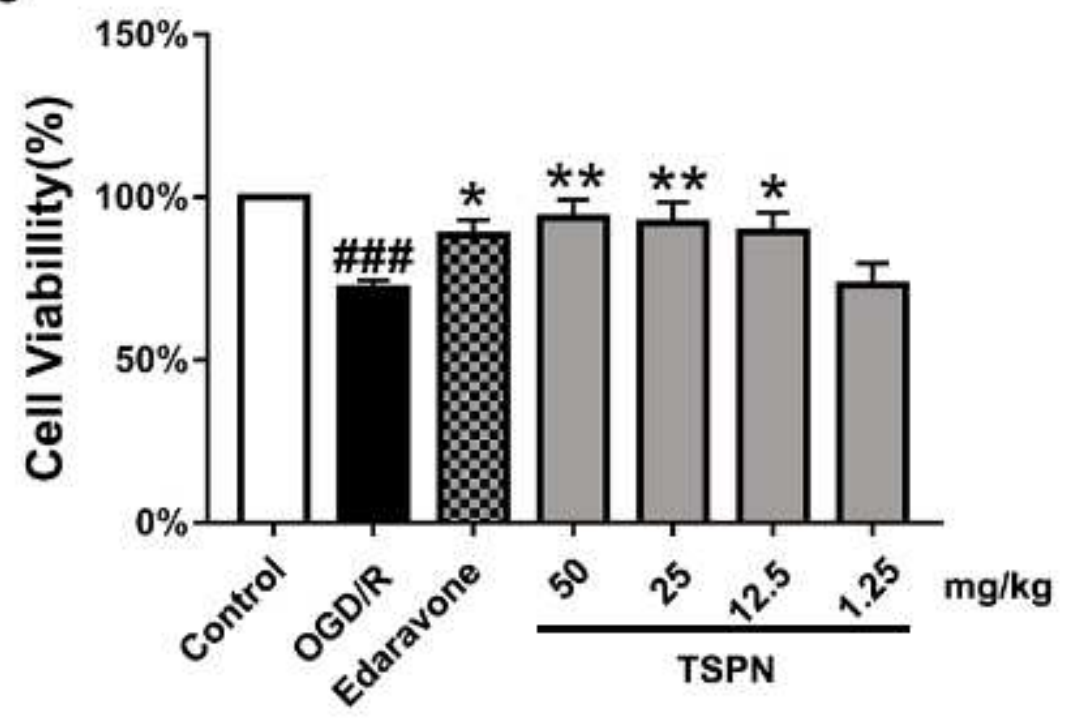

Figure 2

TSPN protect cortical neurons against OGD/R-induced cell injury. (a) Cortical neurons were treated with different concentrations of TSPN and cell viabilities were determined after $24 \mathrm{~h}$. ${ }^{*} \mathrm{P}<0.05,{ }^{*} \mathrm{P}<0.01$ vs control, $n=3$. (b) Different concentrations of TSPN or Edaravone were added in cortical neurons $2 \mathrm{~h}$ before OGD, as well as the whole process of OGD/R. Cell viability was detected by MTT assay. \#\#\#P< 0.001 vs Control. ${ }^{*} \mathrm{P}<0.05,{ }^{\star *} \mathrm{P}<0.01$ vs $\mathrm{OGD} / \mathrm{R}$ group, $\mathrm{n}=3$. The data are expressed as means $\pm \mathrm{SD}$. 
a

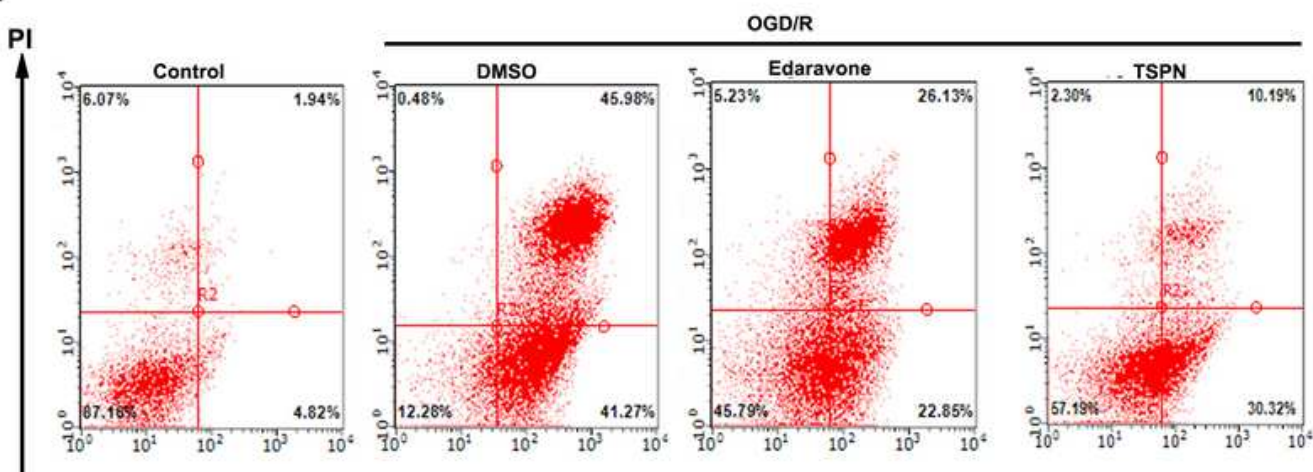

Annexin V

b

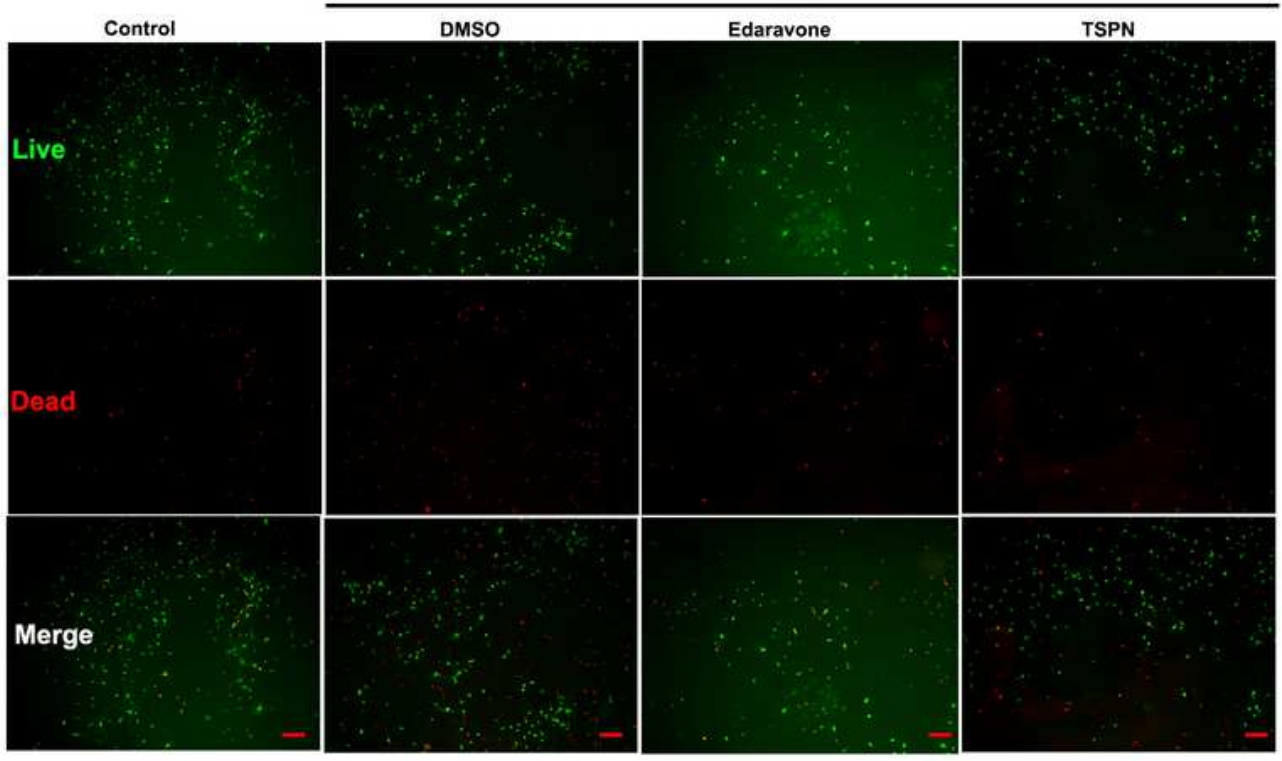

C

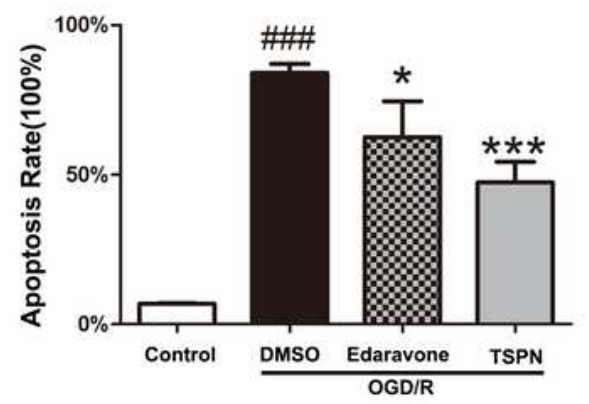

d

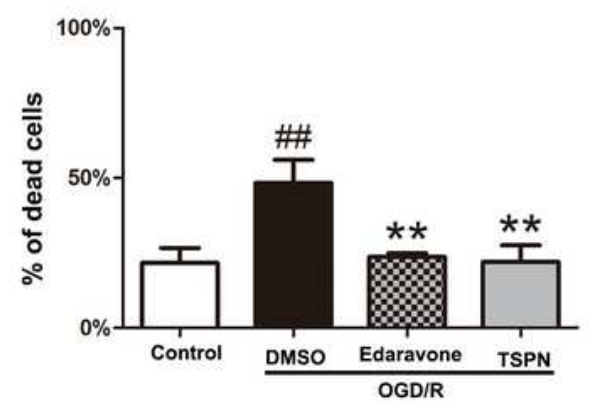

\section{Figure 3}

TSPN reduce OGD/R-induced neuronal apoptosis. (a) Apoptotic cells were detected using Annexin V/PI staining by flow cytometry. (b) The live or dead cells were determined using the Live/Dead cell assay kit $(10 \times$ objective, scale bar $=100 \mu \mathrm{m})$. (c) Cell apoptosis is presented as a combined percentage of Annexin $\mathrm{V}+/ \mathrm{PI}-$ and Annexin V+/PI+ cells. \#\#\#P $<0.001$ vs Control. ${ }^{\mathrm{P}}<0.05,{ }^{\star \star *} \mathrm{P}<0.001$ vs DMSO group. (d) 
The percentage of dead cells was counted. \#\#P $<0.01$ vs Control. ** $\mathrm{P}<0.01$ vs DMSO group, $\mathrm{n}=3$. The data are expressed as means \pm SD.

a
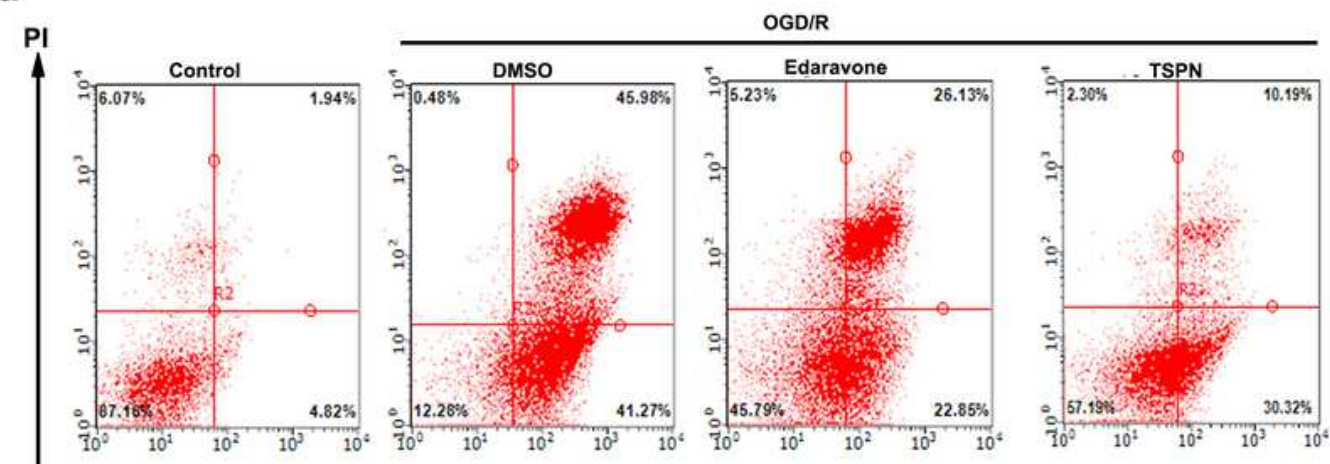

Annexin V

b

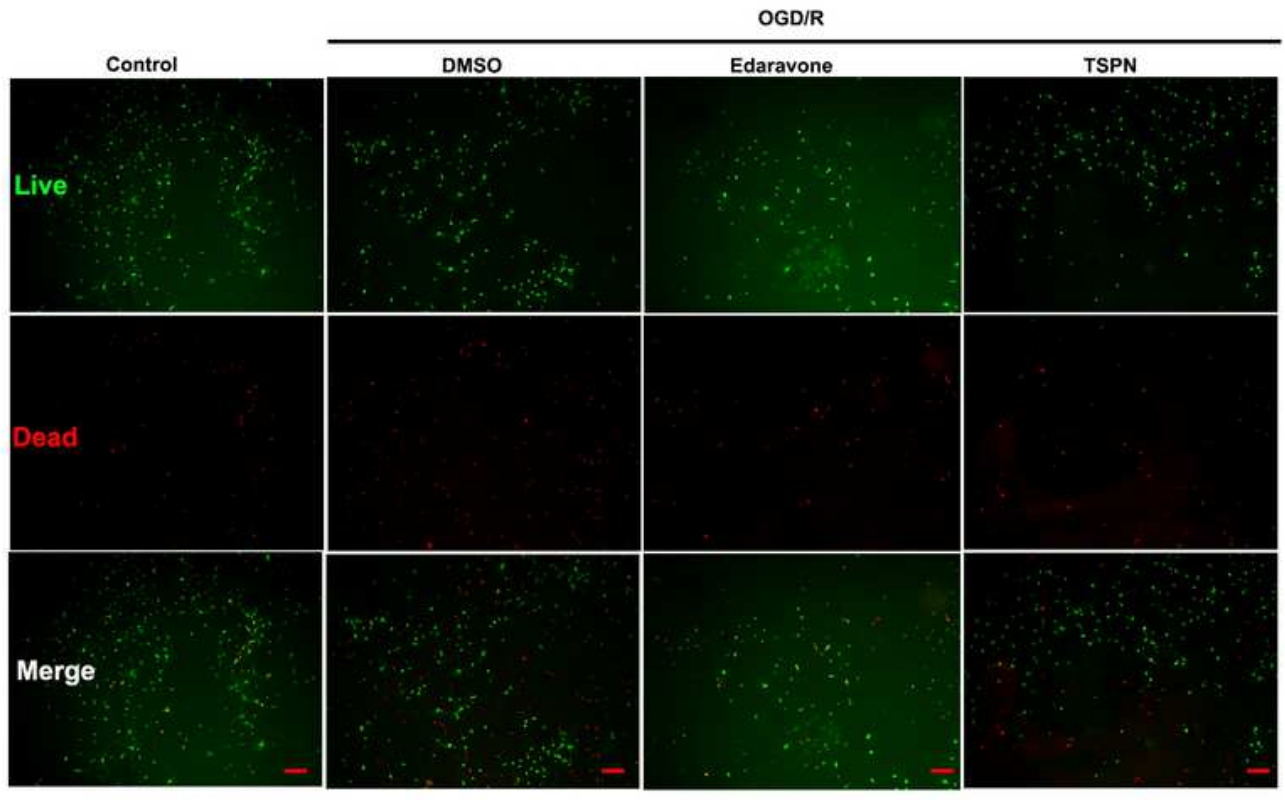

c

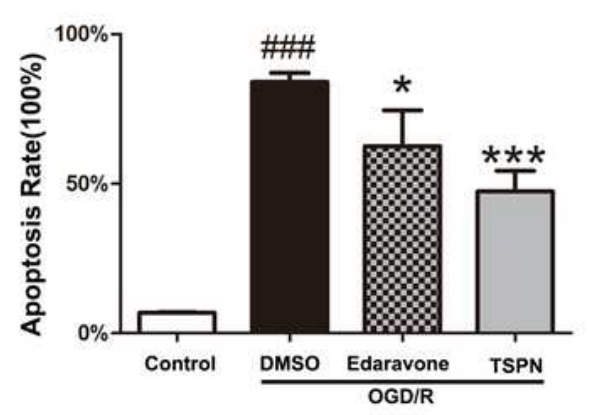

d

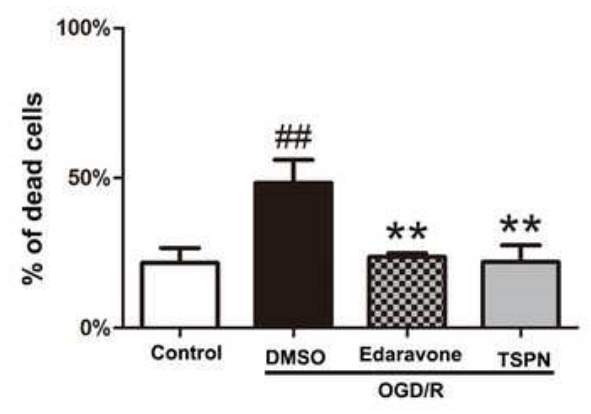

\section{Figure 3}

TSPN reduce OGD/R-induced neuronal apoptosis. (a) Apoptotic cells were detected using Annexin V/PI staining by flow cytometry. (b) The live or dead cells were determined using the Live/Dead cell assay kit $(10 \times$ objective, scale bar $=100 \mu \mathrm{m})$. (c) Cell apoptosis is presented as a combined percentage of Annexin 
V+/PI- and Annexin V+/PI+ cells. \#\#\#P < 0.001 vs Control. *P $<0.05$, ***P $<0.001$ vs DMSO group. (d) The percentage of dead cells was counted. \#\#P $<0.01$ vs Control. **P $<0.01$ vs DMSO group, $n=3$. The data are expressed as means \pm SD.

a

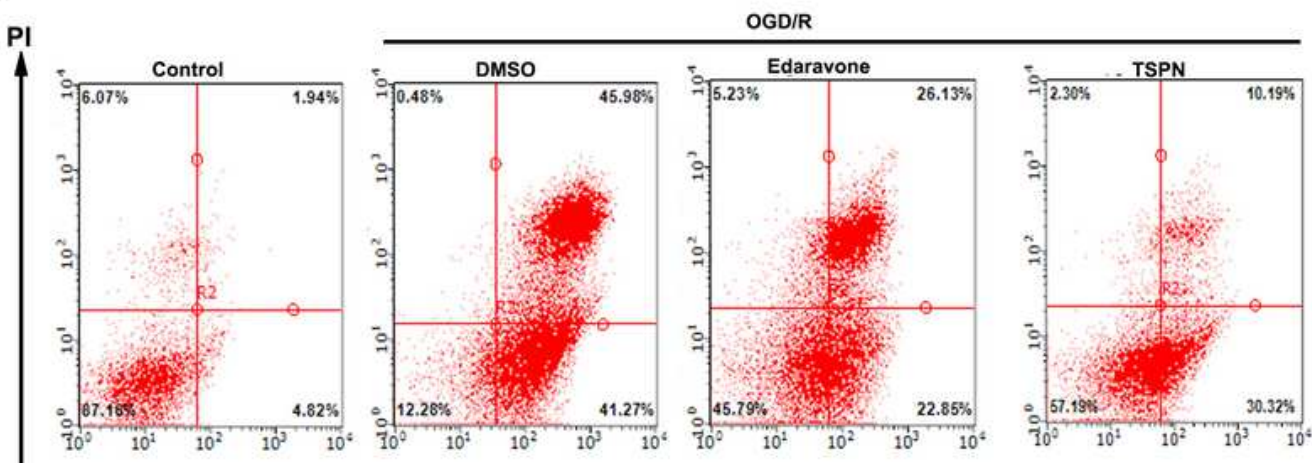

Annexin V

b

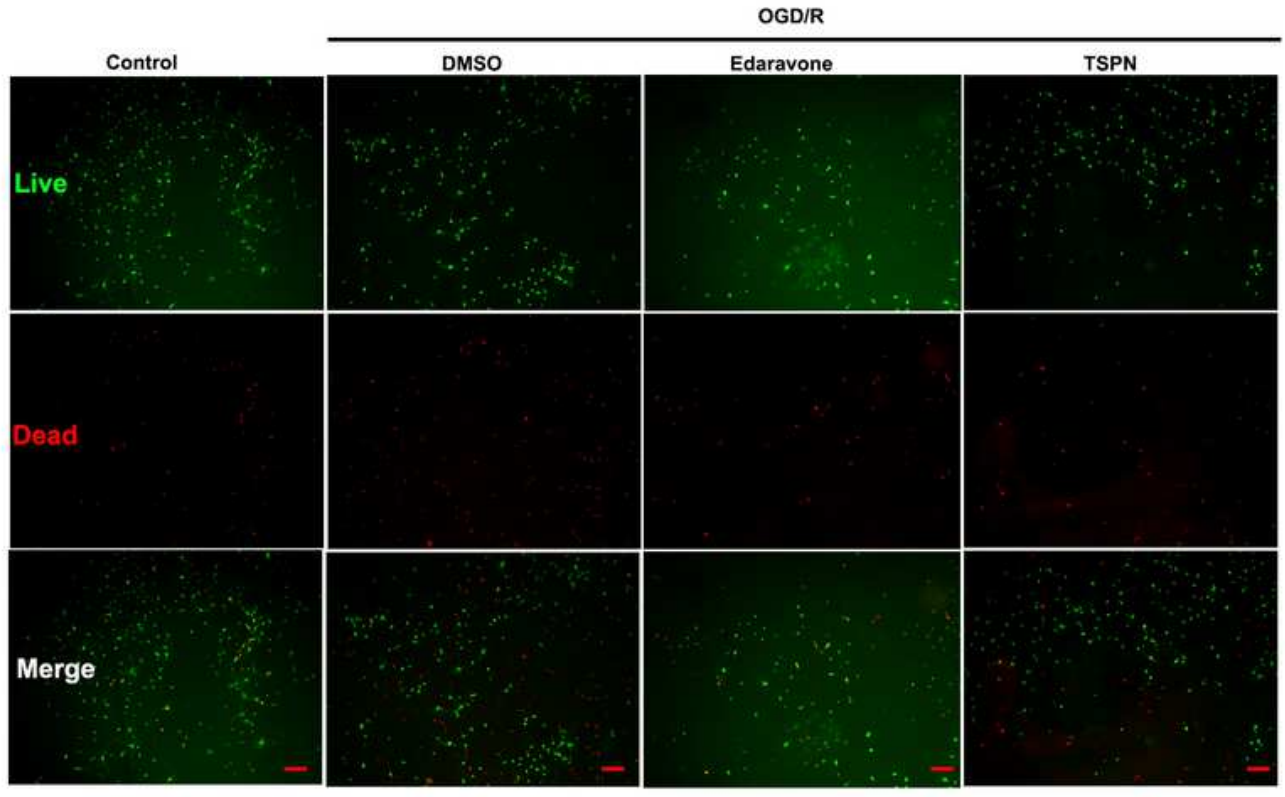

C

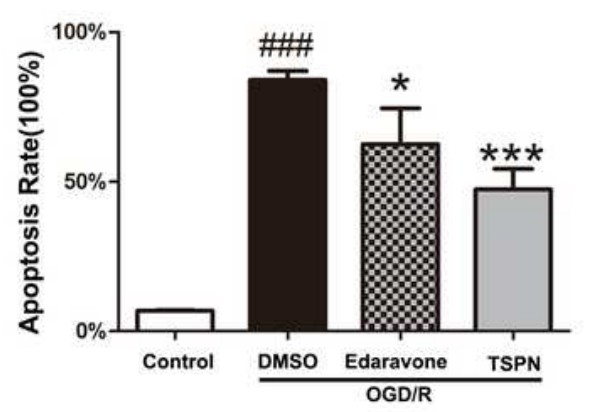

d

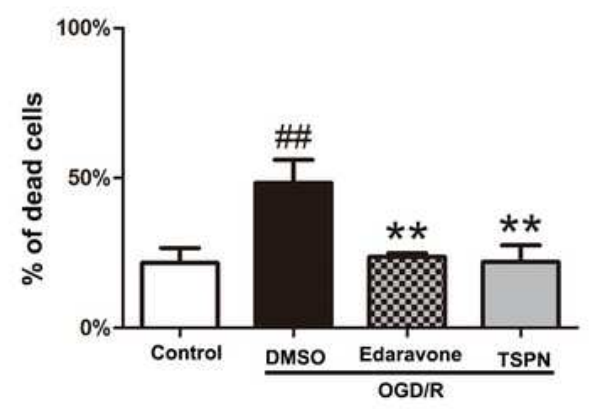

Figure 3

TSPN reduce OGD/R-induced neuronal apoptosis. (a) Apoptotic cells were detected using Annexin V/PI staining by flow cytometry. (b) The live or dead cells were determined using the Live/Dead cell assay kit 
(10x objective, scale bar $=100 \mu \mathrm{m})$. (c) Cell apoptosis is presented as a combined percentage of Annexin $\mathrm{V}+/ \mathrm{PI}-$ and Annexin V+/PI+ cells. \#\#\#P $<0.001$ vs Control. $* \mathrm{P}<0.05$, $* \star * \mathrm{P}<0.001$ vs DMSO group. (d) The percentage of dead cells was counted. \#\#P $<0.01$ vs Control. ** $\mathrm{P}<0.01$ vs DMSO group, $\mathrm{n}=3$. The data are expressed as means \pm SD.

a
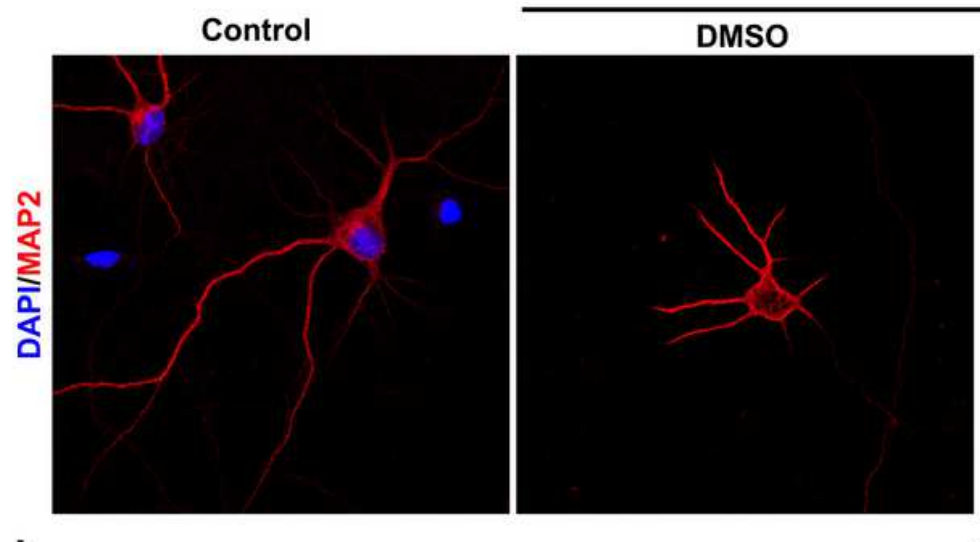

b

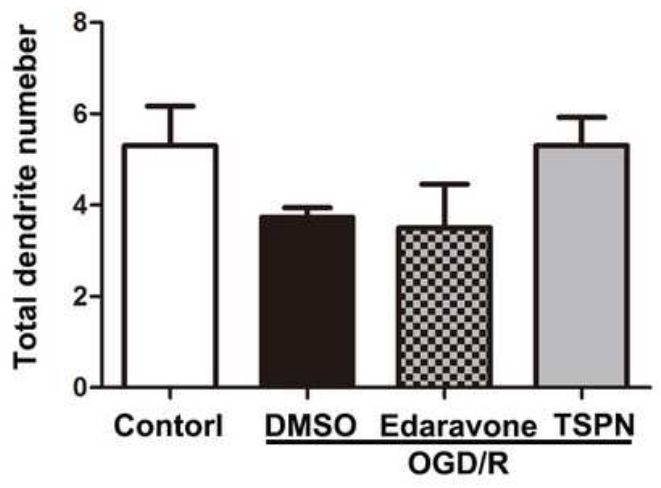

c

OGD/R
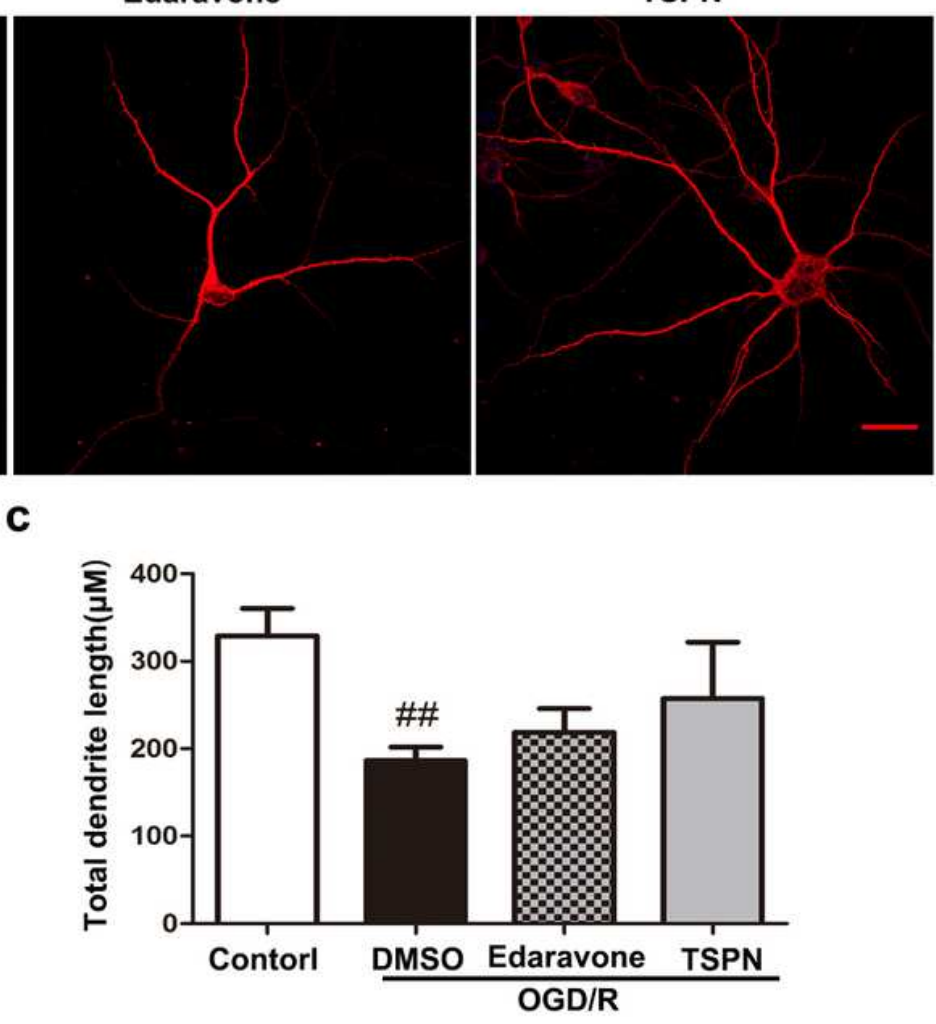

Figure 4

TSPN reverse OGD/R-induced dendritic abnormalities. (a) Dendrites were shown by MAP2 staining (40x objective, scale bar $=20 \mu \mathrm{m}$ ). (b) Total dendrite branches were counted. (c) Total dendrite lengths were measured. \#\#P $<0.01$ vs Control, $n=3$. The data are expressed as means $\pm S D$. 
a

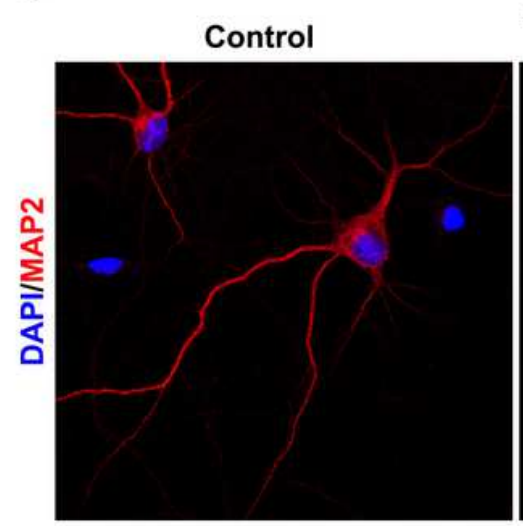

b

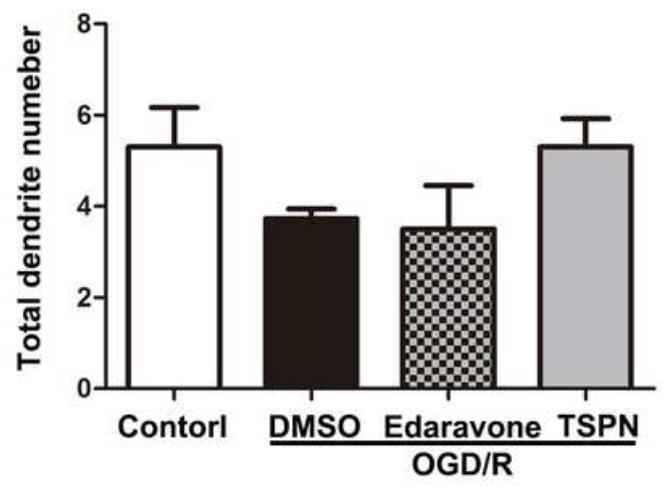

Edaravone
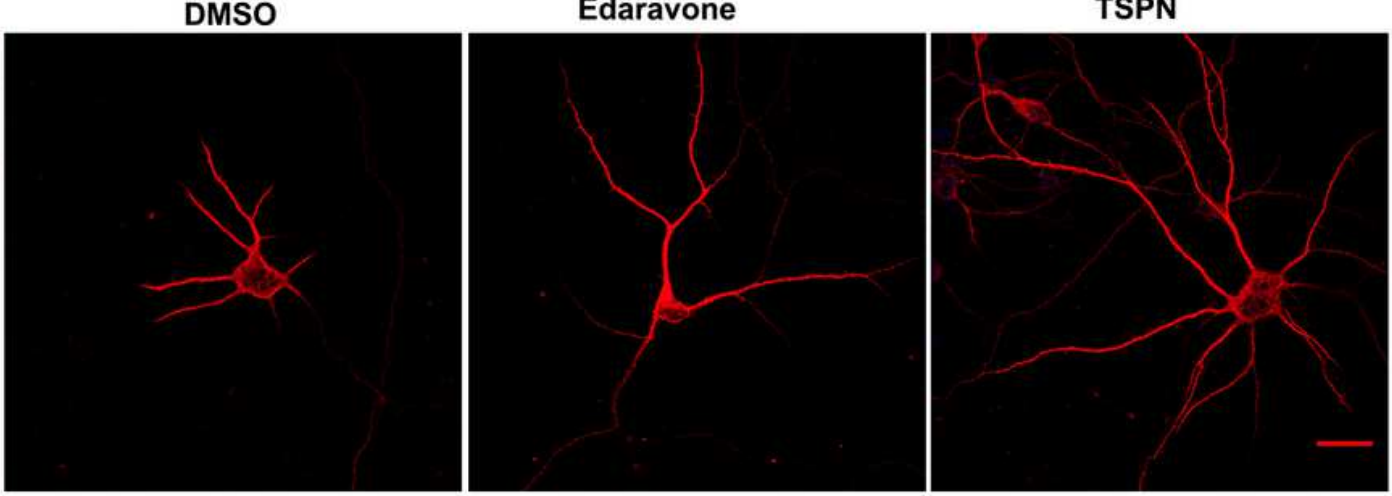

C

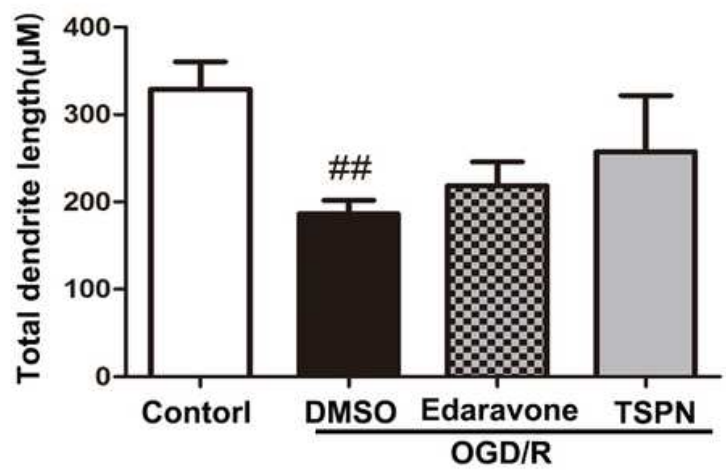

Figure 4

TSPN reverse OGD/R-induced dendritic abnormalities. (a) Dendrites were shown by MAP2 staining (40x objective, scale bar $=20 \mu \mathrm{m}$ ). (b) Total dendrite branches were counted. (c) Total dendrite lengths were measured. \#\#P 0.01 vs Control, $n=3$. The data are expressed as means $\pm S D$. 
a

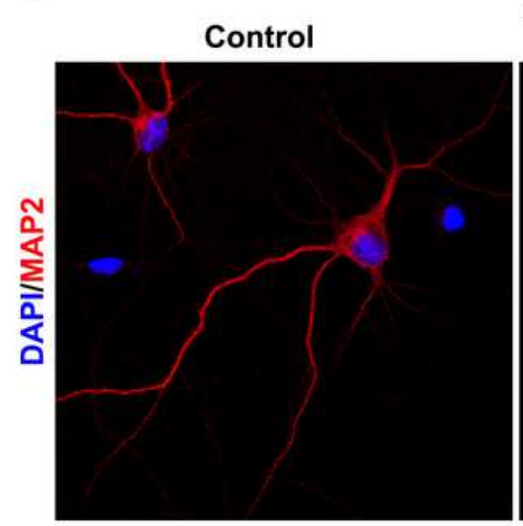

b

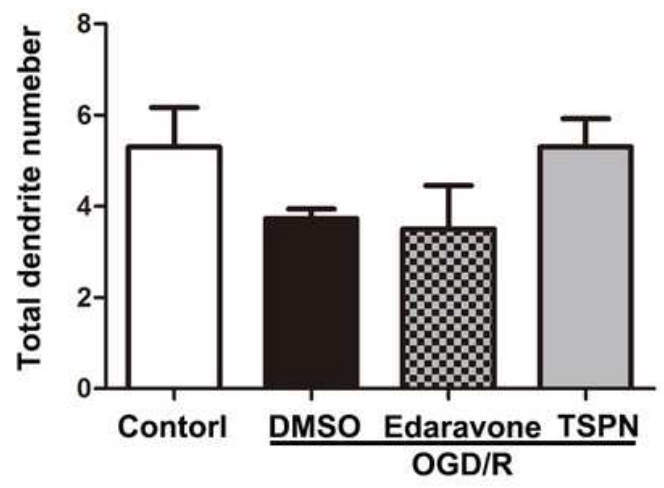

Edaravone
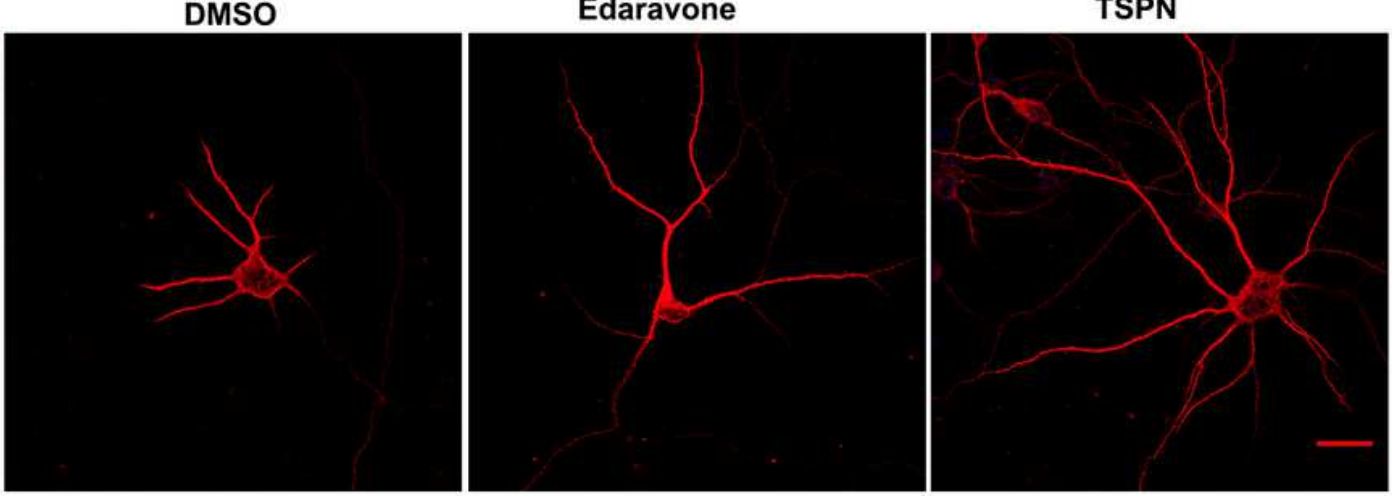

C

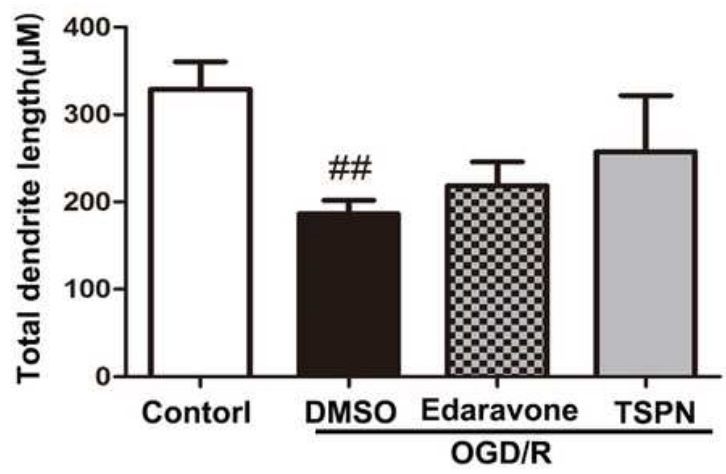

Figure 4

TSPN reverse OGD/R-induced dendritic abnormalities. (a) Dendrites were shown by MAP2 staining (40x objective, scale bar $=20 \mu \mathrm{m}$ ). (b) Total dendrite branches were counted. (c) Total dendrite lengths were measured. \#\#P 0.01 vs Control, $n=3$. The data are expressed as means $\pm S D$. 

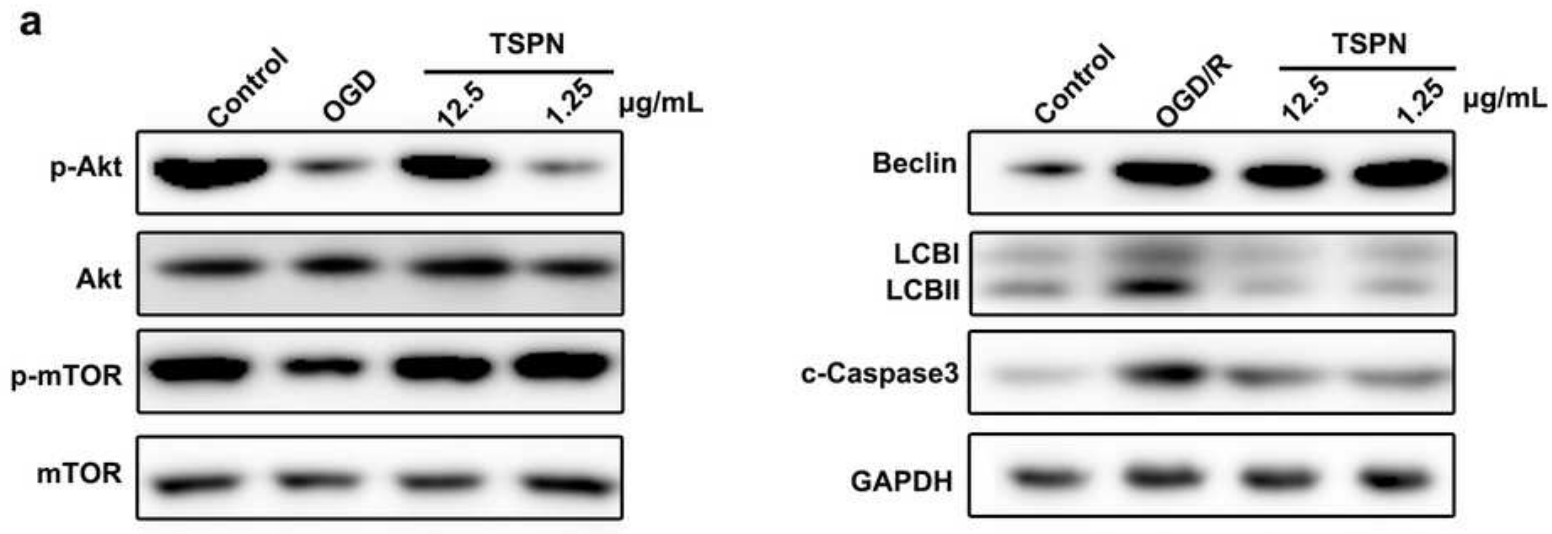

b

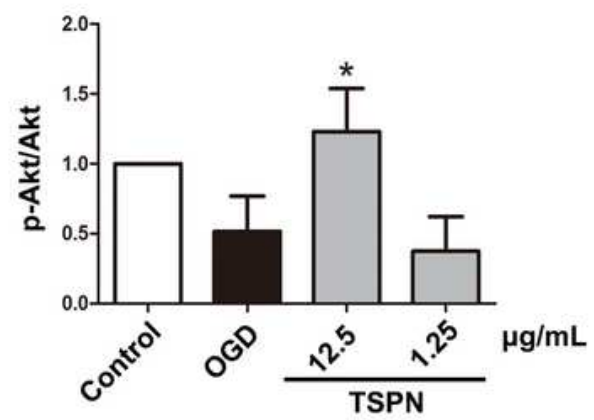

C

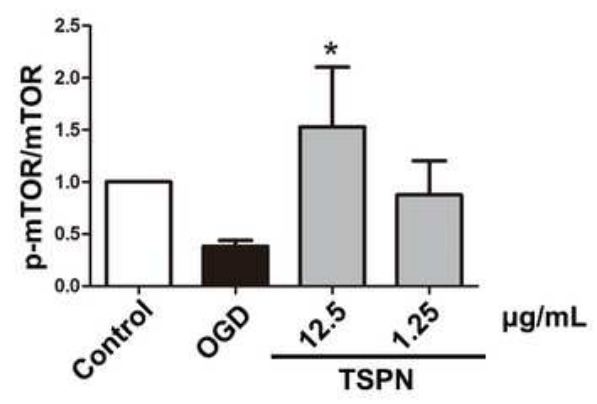

d

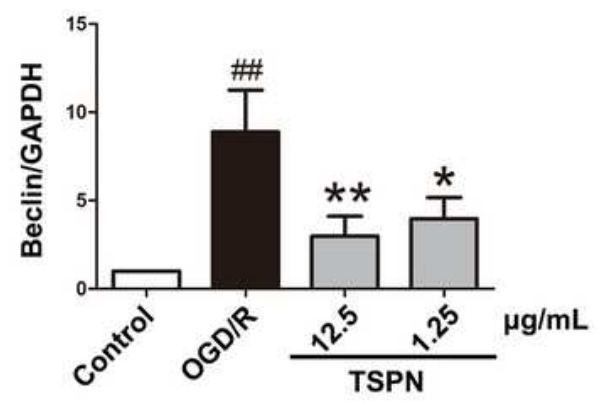

e

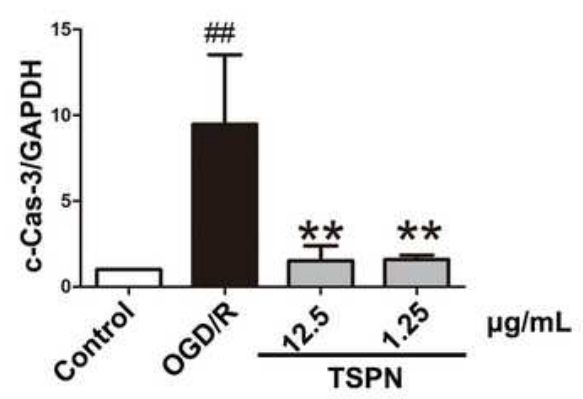

f

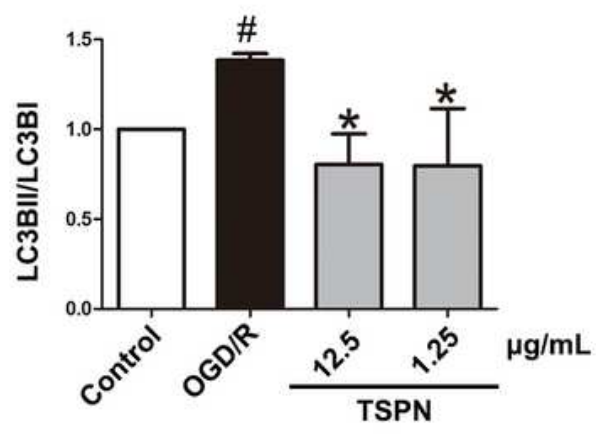

\section{Figure 5}

TSPN restore Akt/mTOR pathway and inhibits the OGD/R-induced pathways involved in autophagy and apoptosis. (a) Levels of p-AKT, p-mTOR, LC3B, Beclin-1, and cleaved-Caspase3 in OGD/R-damaged neurons are reversed by TSPN. (b-f) Relative band density of p-AKT (b), p-mTOR (c), Beclin-1 (d), cleavedCaspase3 (e), and LC3BII (f) were measured using ImageJ. \#P<0.05 vs control, ${ }^{*}<<0.05,{ }^{\star *} P<0.01$ vs $O G D / R$ group, $n=3$, The data are expressed as means $\pm S D$. 

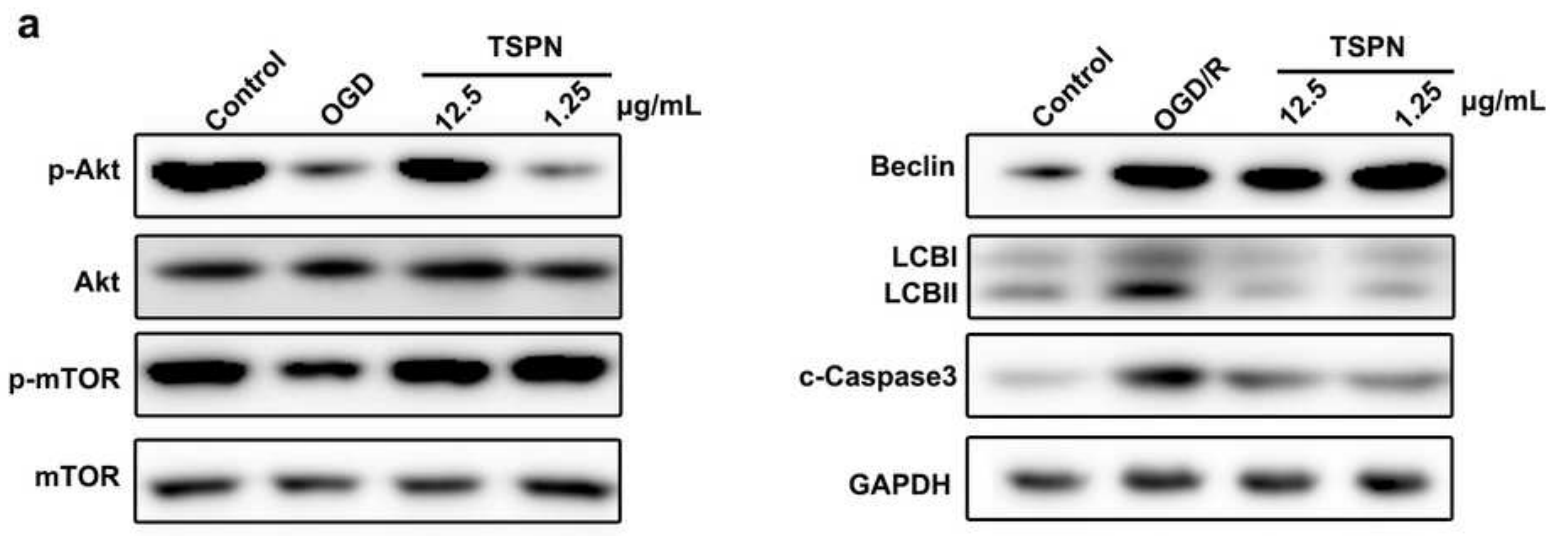

b

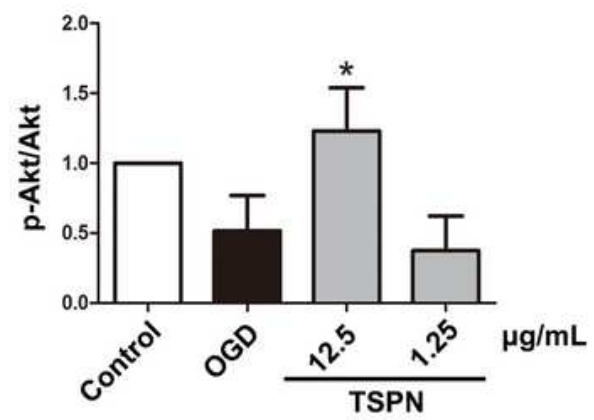

c

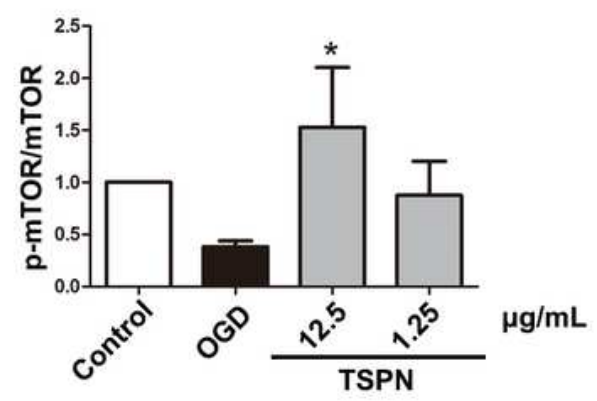

d

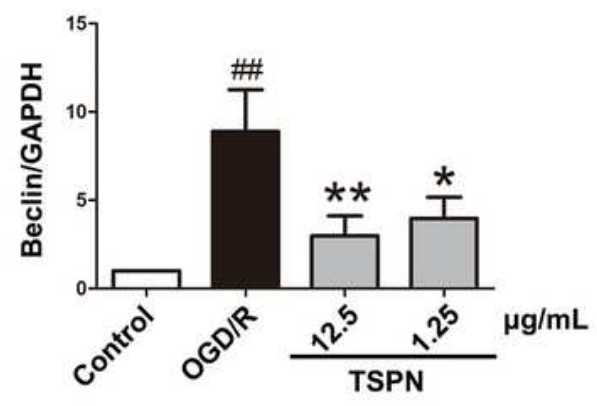

e

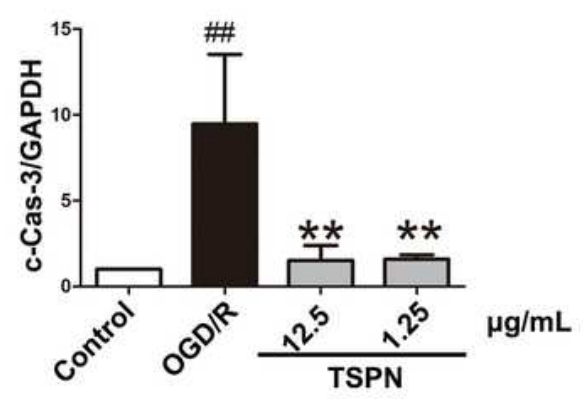

f

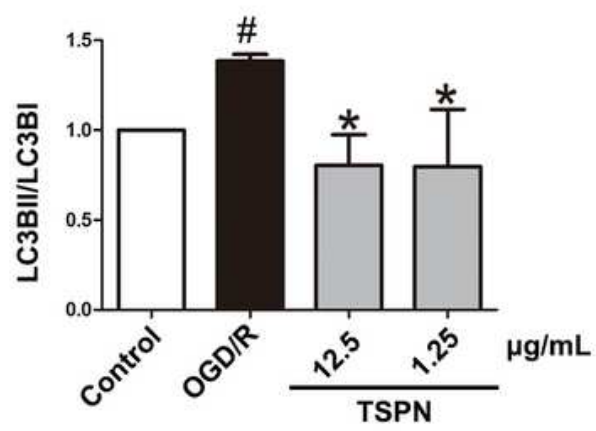

\section{Figure 5}

TSPN restore Akt/mTOR pathway and inhibits the OGD/R-induced pathways involved in autophagy and apoptosis. (a) Levels of p-AKT, p-mTOR, LC3B, Beclin-1, and cleaved-Caspase3 in OGD/R-damaged neurons are reversed by TSPN. (b-f) Relative band density of p-AKT (b), p-mTOR (c), Beclin-1 (d), cleavedCaspase3 (e), and LC3BII (f) were measured using ImageJ. \#P<0.05 vs control, ${ }^{*}<<0.05,{ }^{\star *} P<0.01$ vs OGD/R group, $n=3$, The data are expressed as means $\pm S D$. 

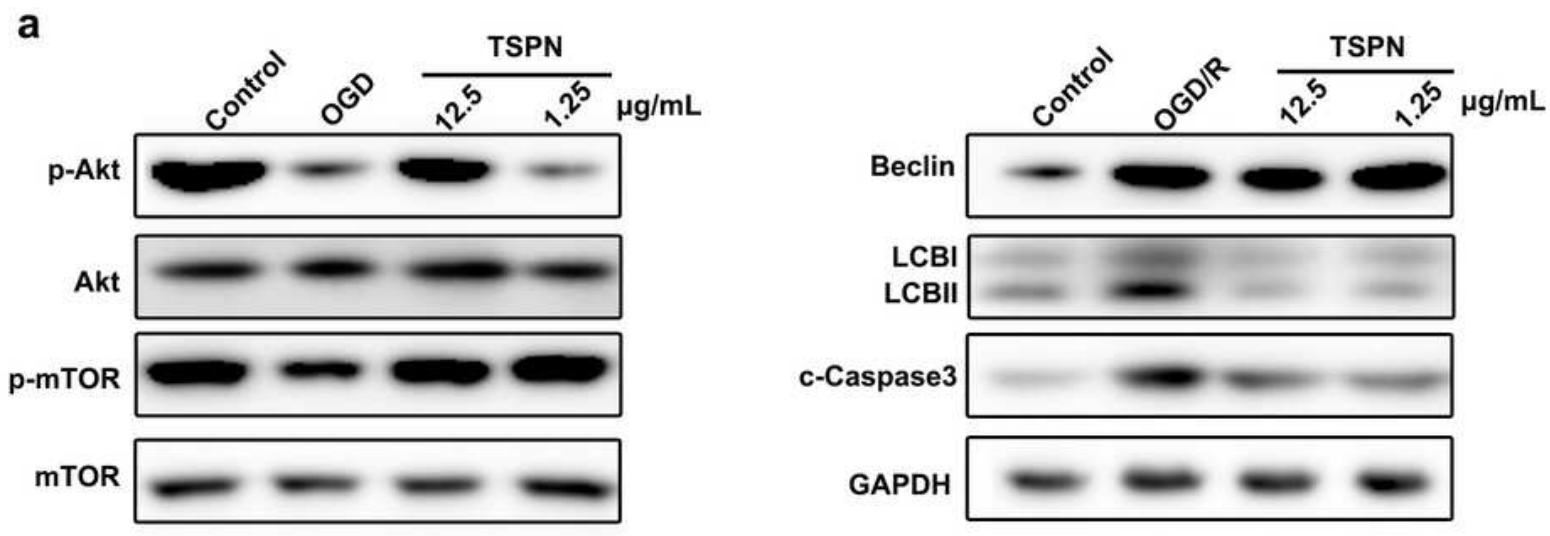

b

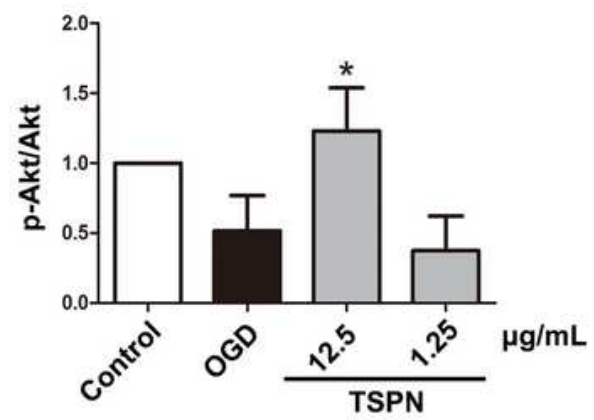

c

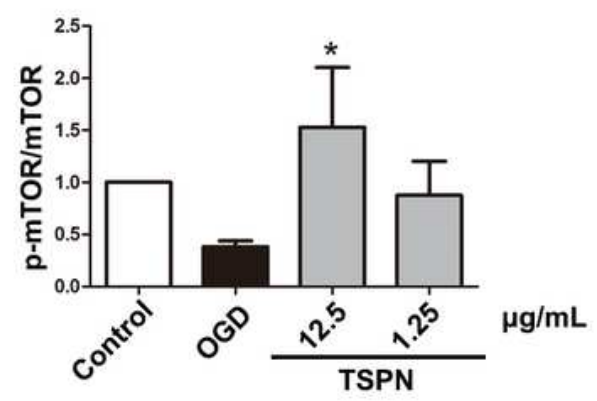

d

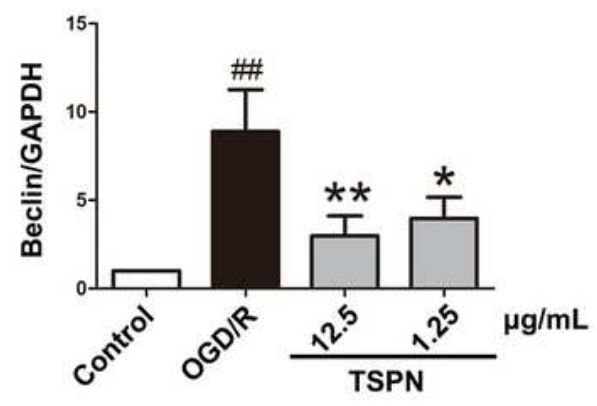

e

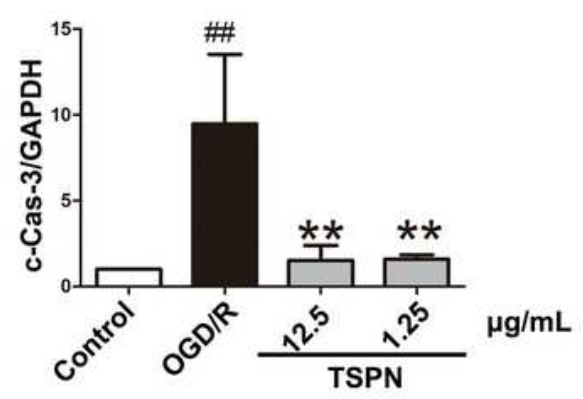

f

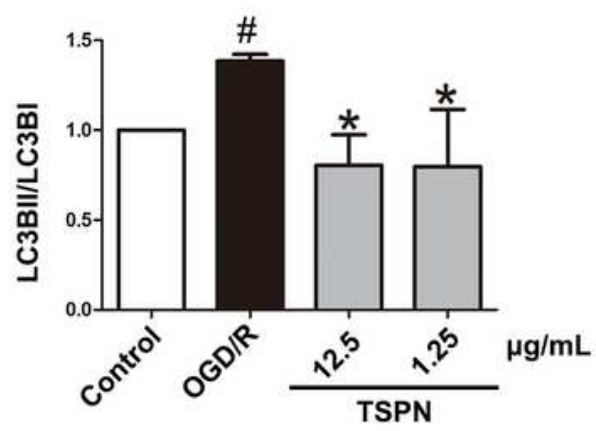

\section{Figure 5}

TSPN restore Akt/mTOR pathway and inhibits the OGD/R-induced pathways involved in autophagy and apoptosis. (a) Levels of p-AKT, p-mTOR, LC3B, Beclin-1, and cleaved-Caspase3 in OGD/R-damaged neurons are reversed by TSPN. (b-f) Relative band density of p-AKT (b), p-mTOR (c), Beclin-1 (d), cleavedCaspase3 (e), and LC3BII (f) were measured using ImageJ. \#P<0.05 vs control, ${ }^{*}<<0.05,{ }^{\star *} P<0.01$ vs OGD/R group, $n=3$, The data are expressed as means $\pm S D$. 
a

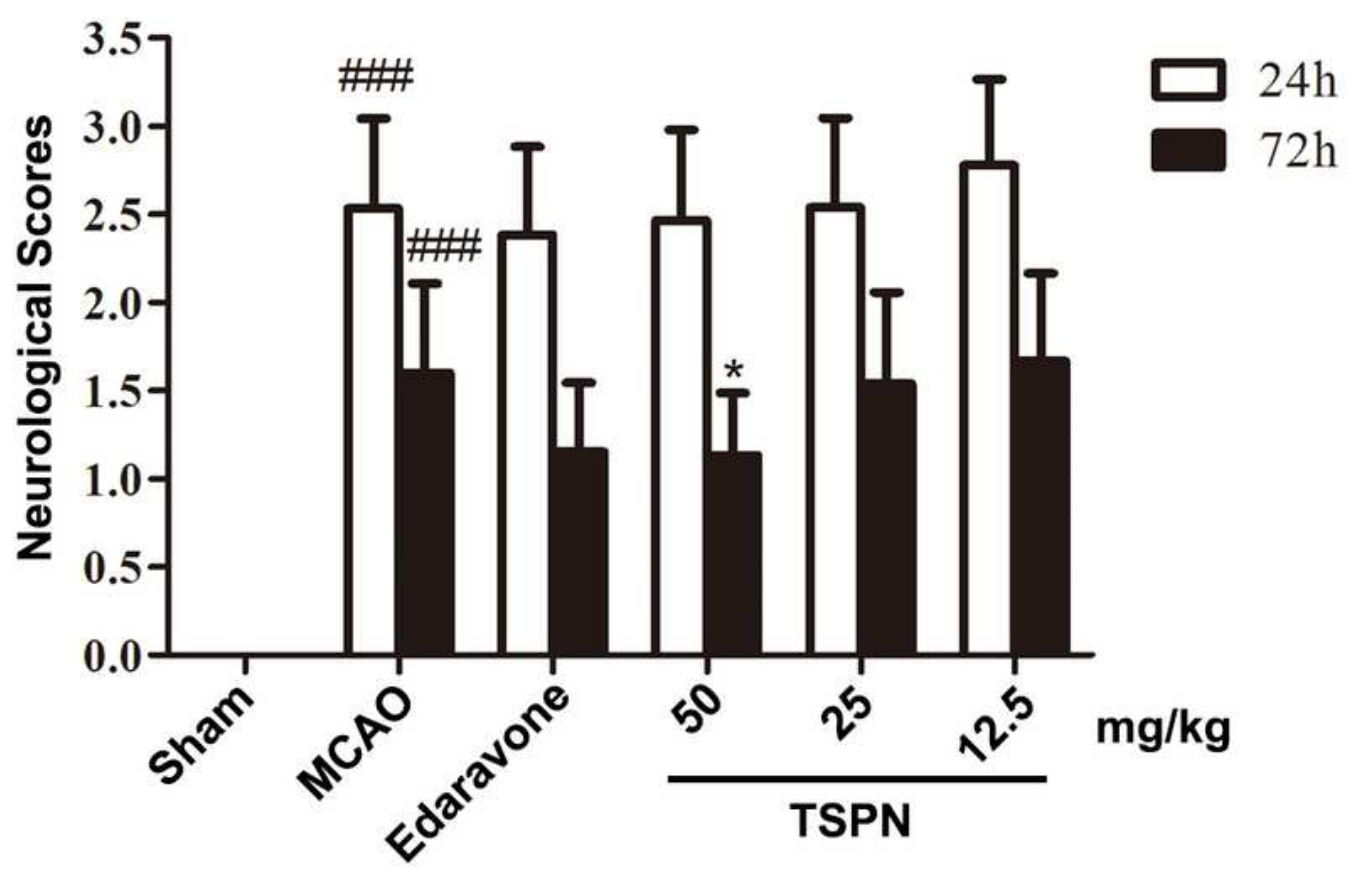

b

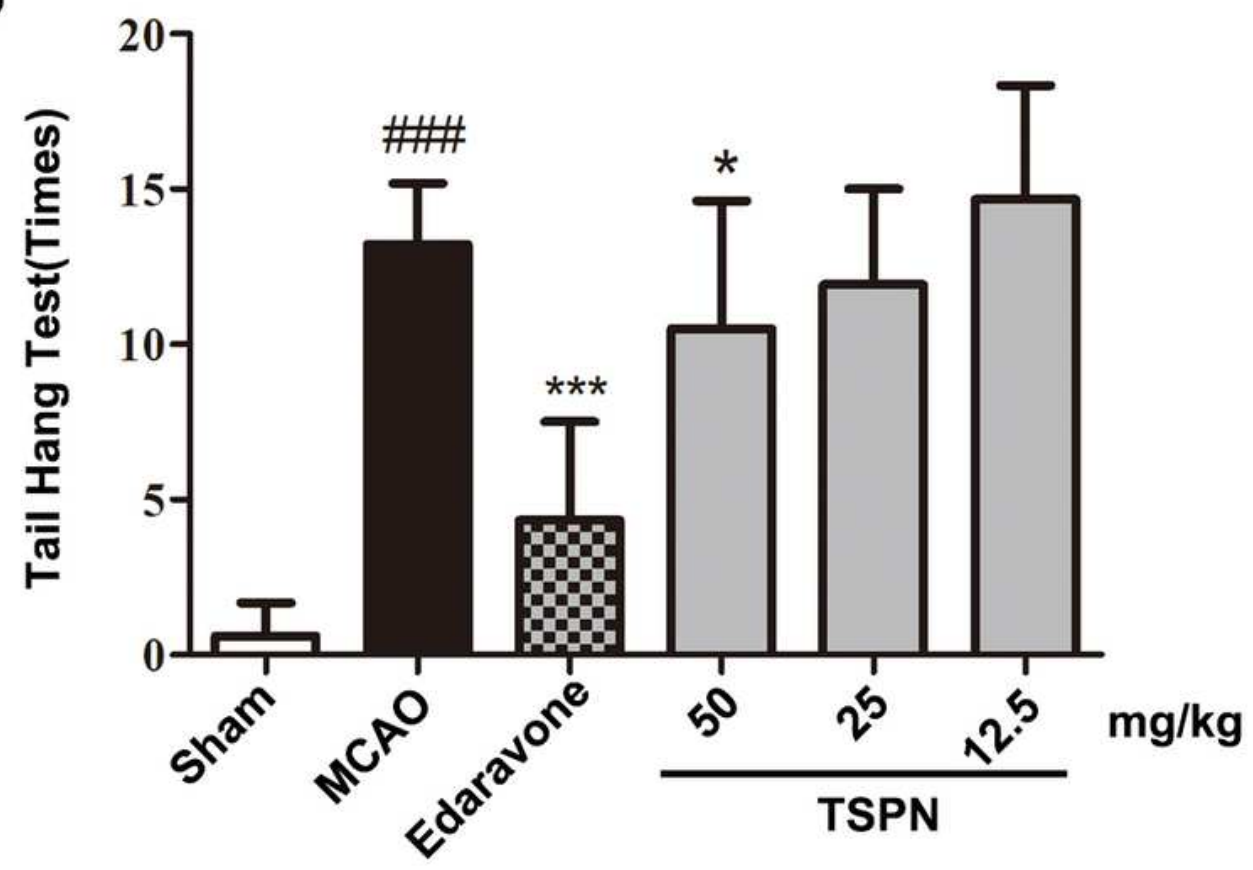

Figure 6

TSPN improve the rats motor function at $72 \mathrm{~h}$ after reperfusion. (a) Neurological score of rats at 24 and $72 \mathrm{~h}$ after ischemia-reperfusion. \#\#\#P $<0.001$ vs Sham group, ${ }^{*} P<0.05$ vs MCAO group, $n=6$. Analyzed using two-way ANOVA. (b) Head rotation frequency at $72 \mathrm{~h}$ after cerebral ischemia-reperfusion. \#\#\# < 0.001 vs Sham group, ${ }^{*}<0.05$, ${ }^{\star \star \star} P<0.001$ vs $M C A O$ group, $n=6$. The data are expressed as means \pm SD. 
a

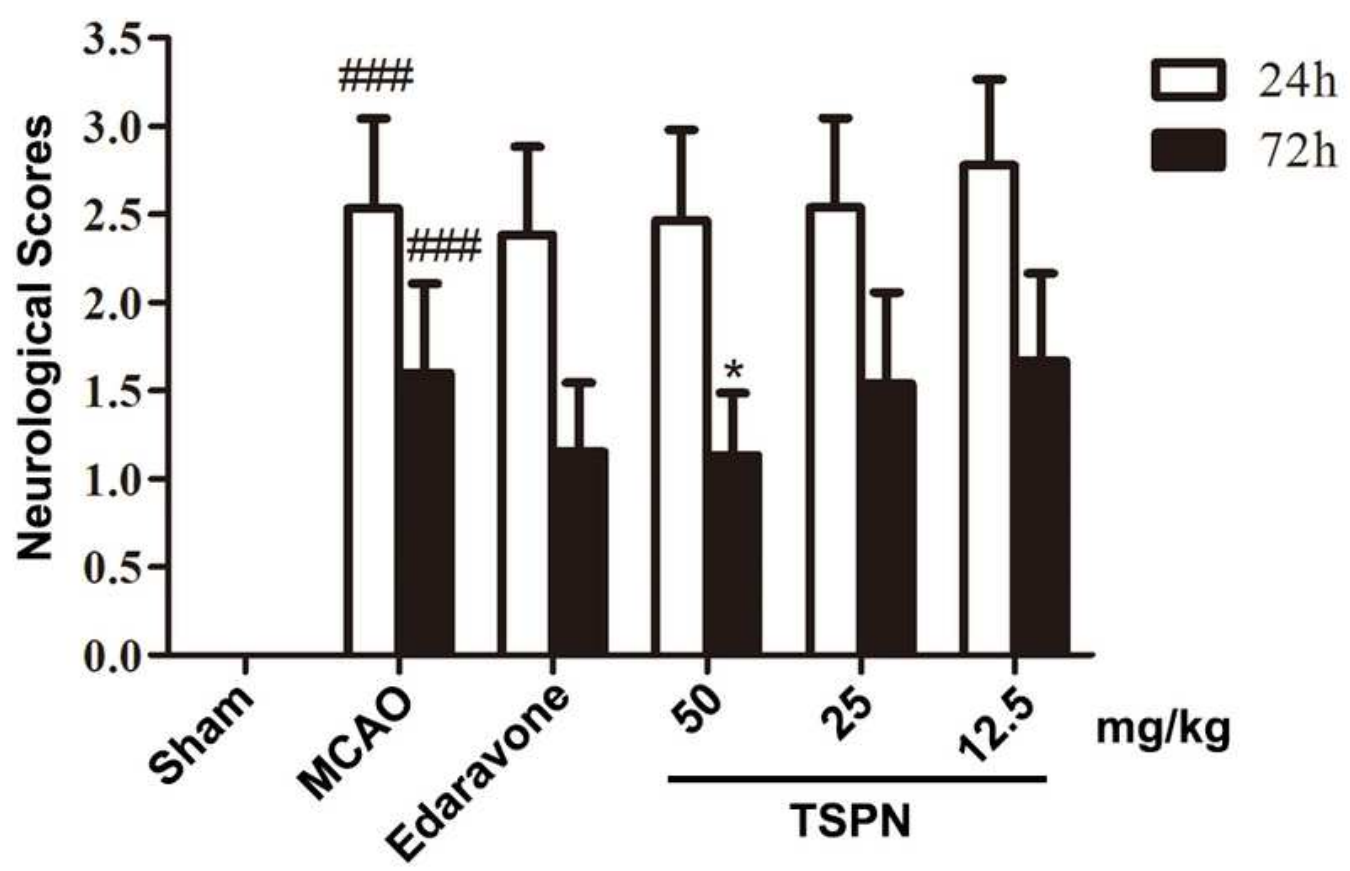

b

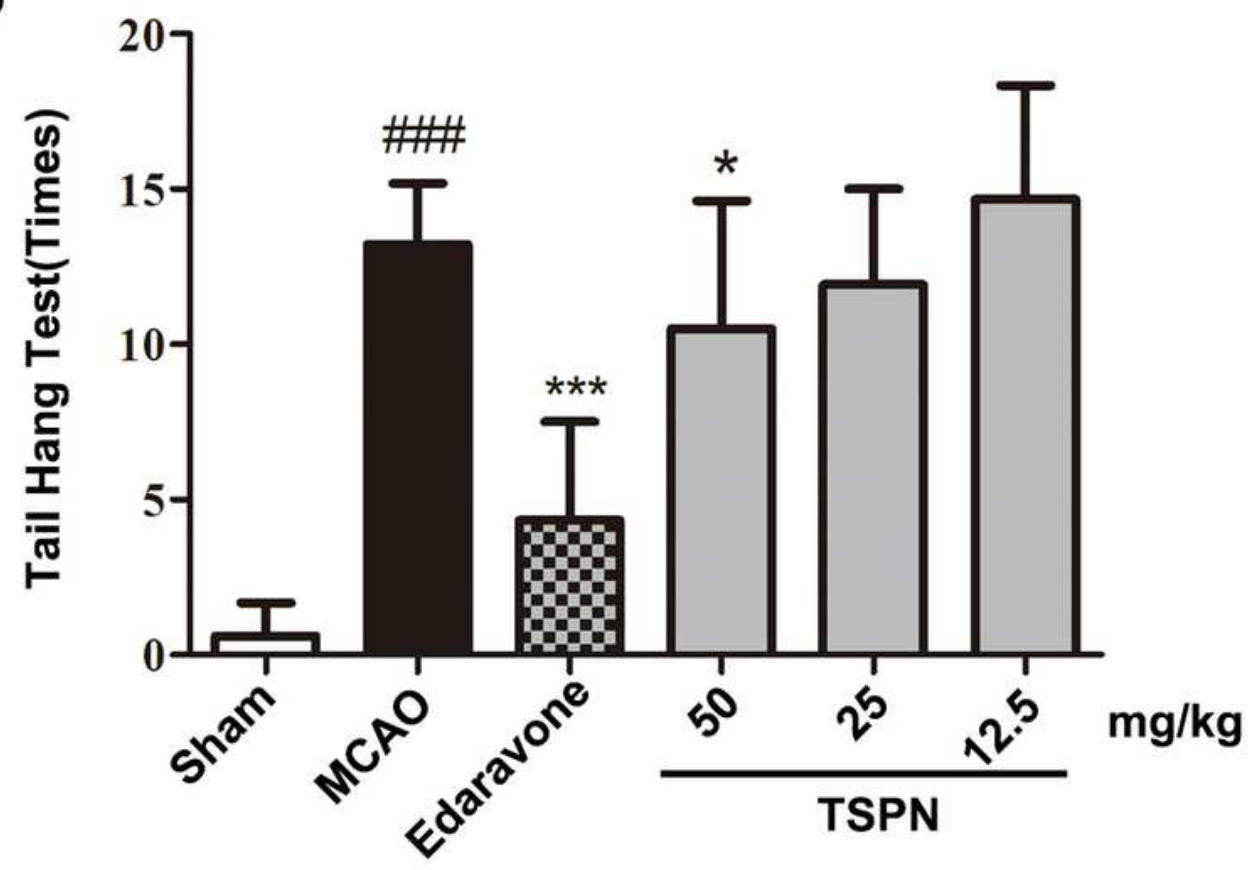

Figure 6

TSPN improve the rats motor function at $72 \mathrm{~h}$ after reperfusion. (a) Neurological score of rats at 24 and $72 \mathrm{~h}$ after ischemia-reperfusion. \#\#\#P $<0.001$ vs Sham group, ${ }^{*} P<0.05$ vs MCAO group, $n=6$. Analyzed using two-way ANOVA. (b) Head rotation frequency at $72 \mathrm{~h}$ after cerebral ischemia-reperfusion. \#\#\# < 0.001 vs Sham group, ${ }^{*}<0.05$, ${ }^{\star \star \star} P<0.001$ vs $M C A O$ group, $n=6$. The data are expressed as means \pm SD. 
a

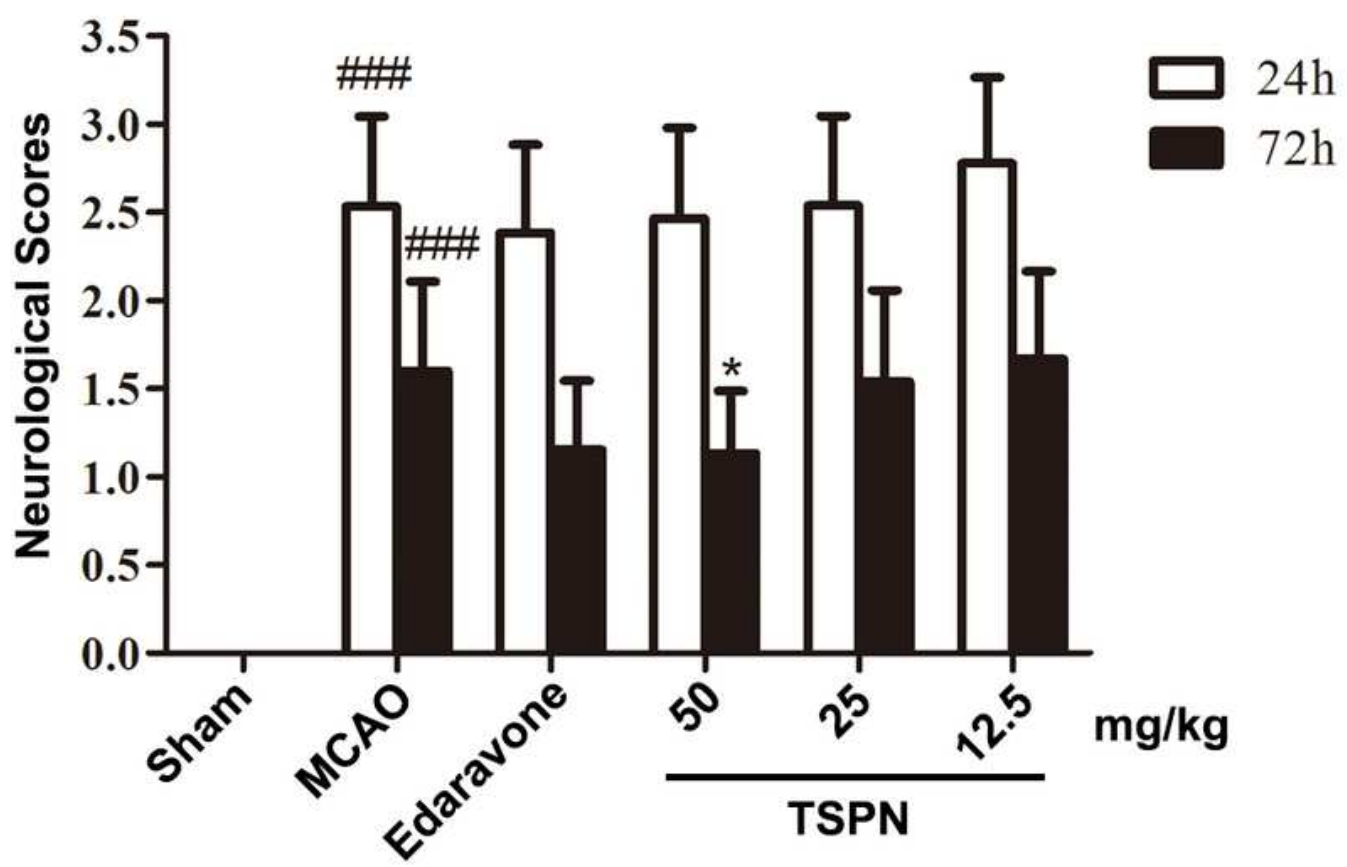

b

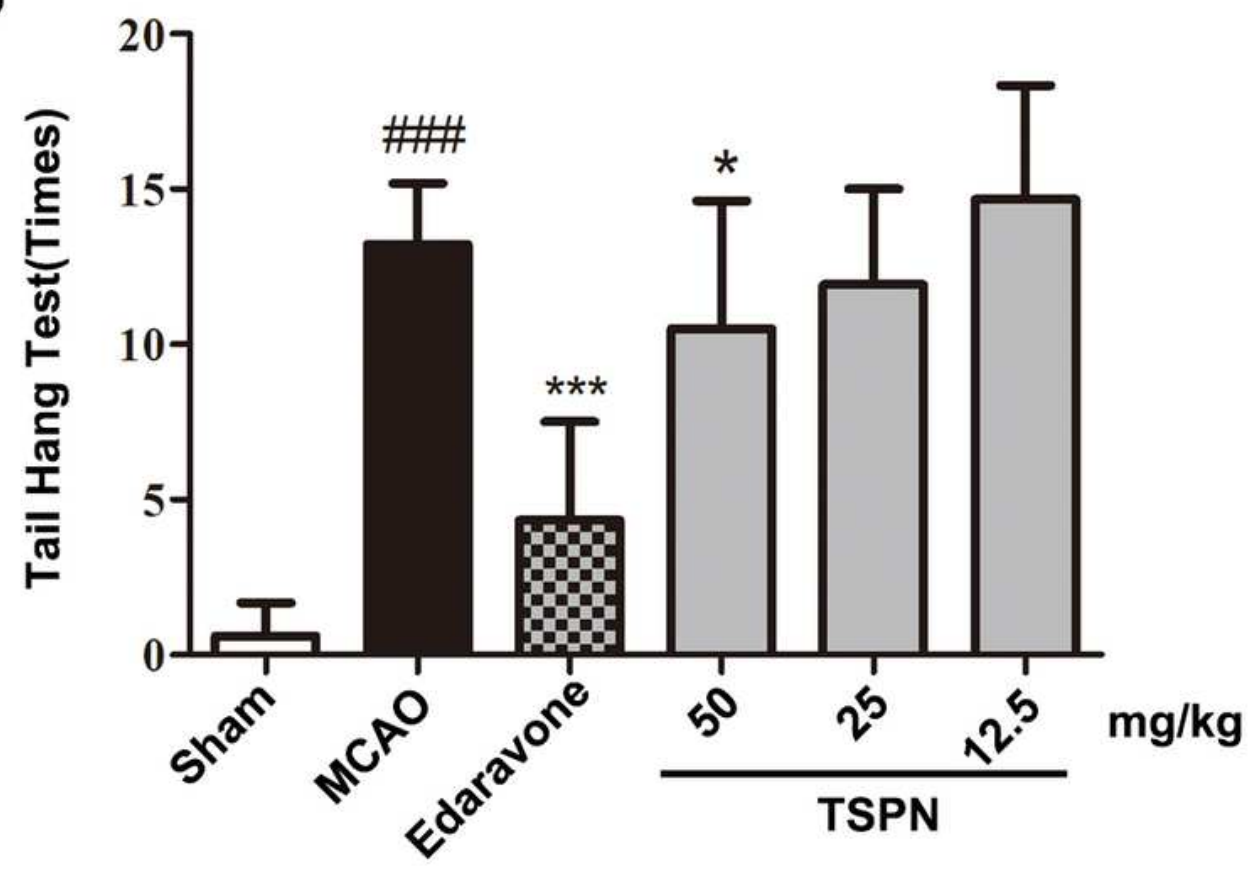

Figure 6

TSPN improve the rats motor function at $72 \mathrm{~h}$ after reperfusion. (a) Neurological score of rats at 24 and $72 \mathrm{~h}$ after ischemia-reperfusion. \#\#\#P $<0.001$ vs Sham group, ${ }^{*} P<0.05$ vs MCAO group, $n=6$. Analyzed using two-way ANOVA. (b) Head rotation frequency at $72 \mathrm{~h}$ after cerebral ischemia-reperfusion. \#\#\# < 0.001 vs Sham group, ${ }^{*}<0.05$, ${ }^{\star \star \star} P<0.001$ vs $M C A O$ group, $n=6$. The data are expressed as means \pm SD. 
a

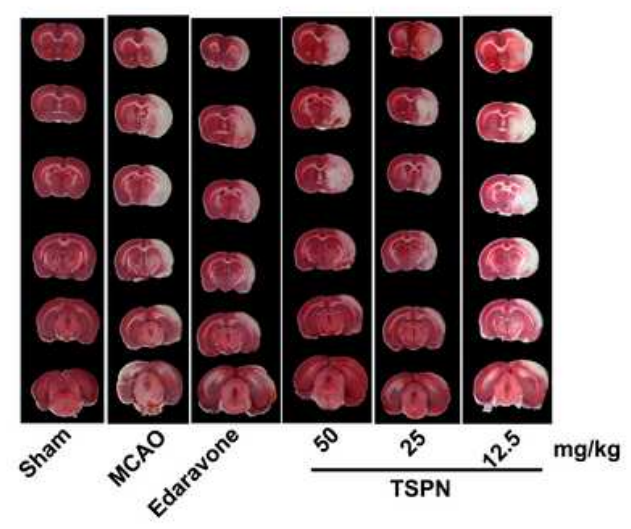

c

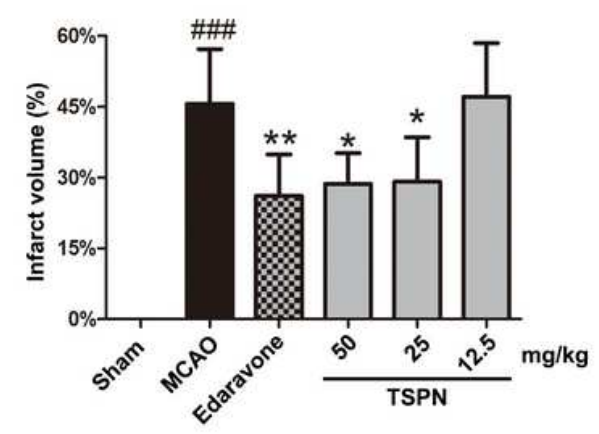

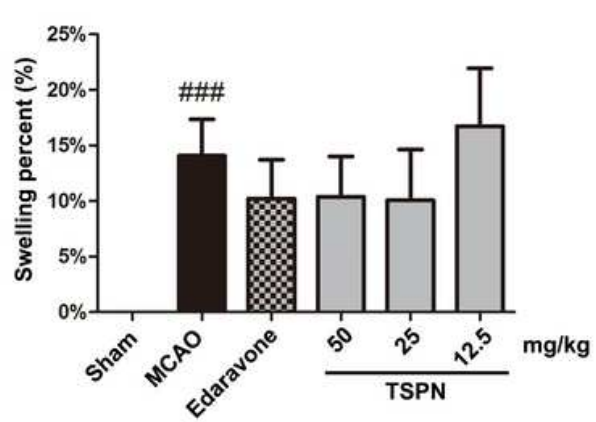

Figure 7

TSPN reduce infarction resulted from cerebral ischemia in MCAO rats. (a) TTC staining was used to detect cerebral infarction in rats of each group. (b) The infarct volume was determined by using ImagePro Plus. (c) Statistical results of brain edema volume in each group. \#\#\#P $<0.001$ vs Sham group, $* \mathrm{P}<$ $0.05,{ }^{*} \mathrm{P}<0.01$ vs MCAO group, $\mathrm{n}=6$. The data are expressed as means $\pm \mathrm{SD}$.

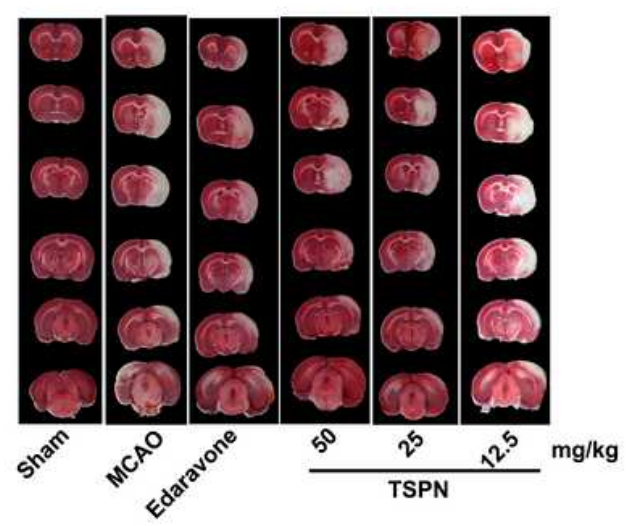

b

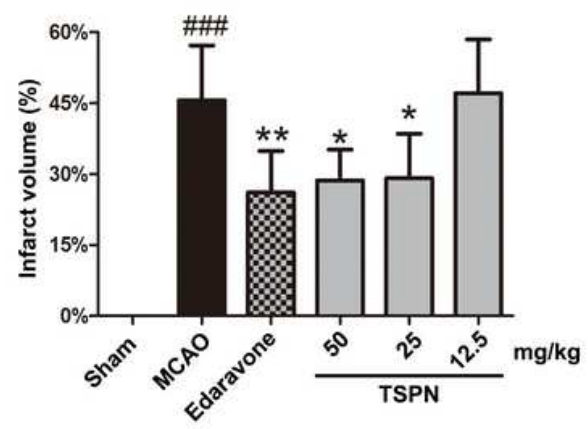

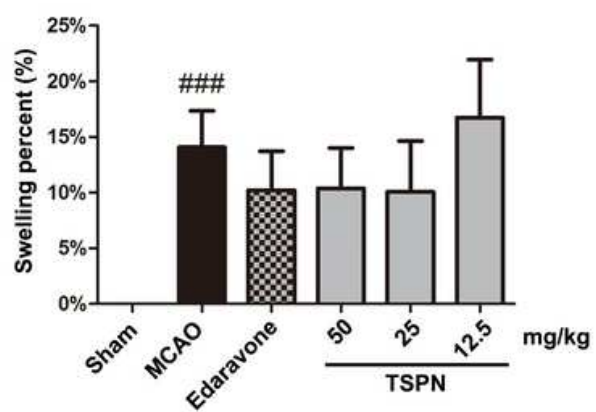

Figure 7

TSPN reduce infarction resulted from cerebral ischemia in MCAO rats. (a) TTC staining was used to detect cerebral infarction in rats of each group. (b) The infarct volume was determined by using ImagePro Plus. (c) Statistical results of brain edema volume in each group. \#\#\#P $<0.001$ vs Sham group, ${ }^{*} \mathrm{P}$ $0.05, \star \star P<0.01$ vs MCAO group, $\mathrm{n}=6$. The data are expressed as means $\pm \mathrm{SD}$. 
a

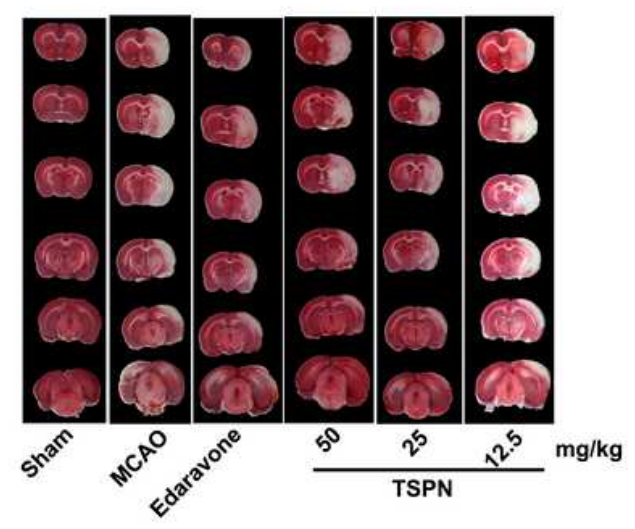

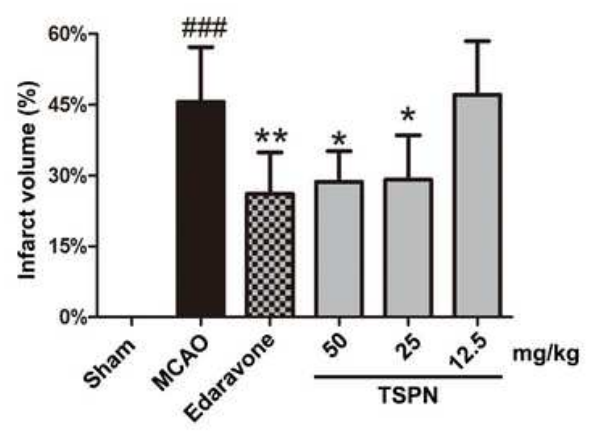

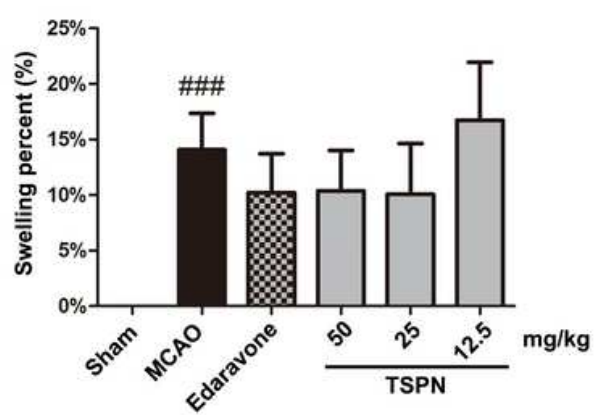

Figure 7

TSPN reduce infarction resulted from cerebral ischemia in MCAO rats. (a) TTC staining was used to detect cerebral infarction in rats of each group. (b) The infarct volume was determined by using ImagePro Plus. (c) Statistical results of brain edema volume in each group. \#\#\#P 0.001 vs Sham group, ${ }^{\mathrm{P}}<$ $0.05,{ }^{*} \mathrm{P}<0.01$ vs MCAO group, $\mathrm{n}=6$. The data are expressed as means $\pm \mathrm{SD}$.

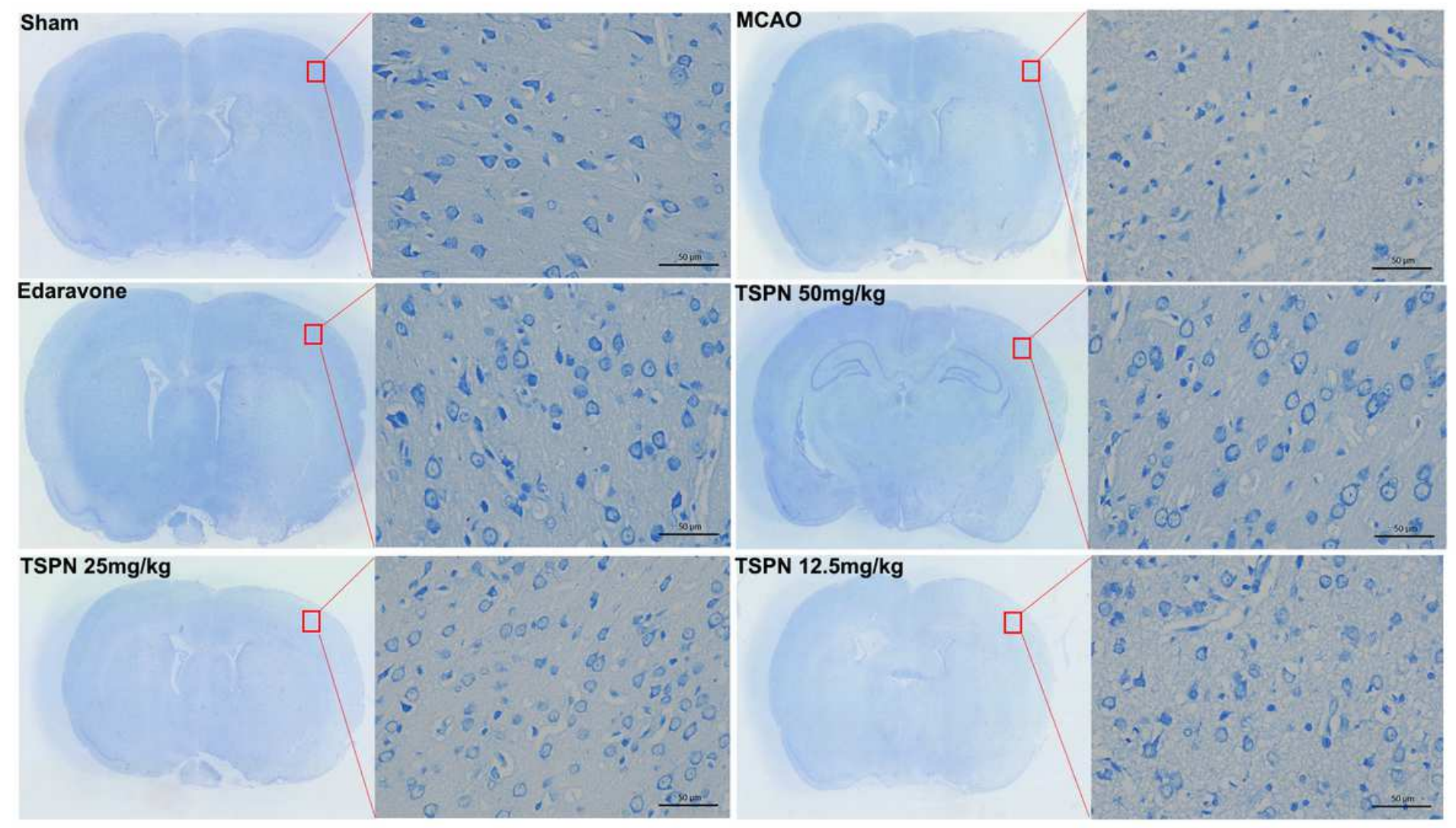

\section{Figure 8}

Nissl staining of ischemic penumbra (400x, scale bar $=50 \mu \mathrm{m}, \mathrm{n}=7)$ There was a big difference in morphology between normal neurons and abnormal neurons. The former had normal morphology, clear nucleolus, abundant cytoplasm and intact structure. The latter had shrinkage and deep staining. 


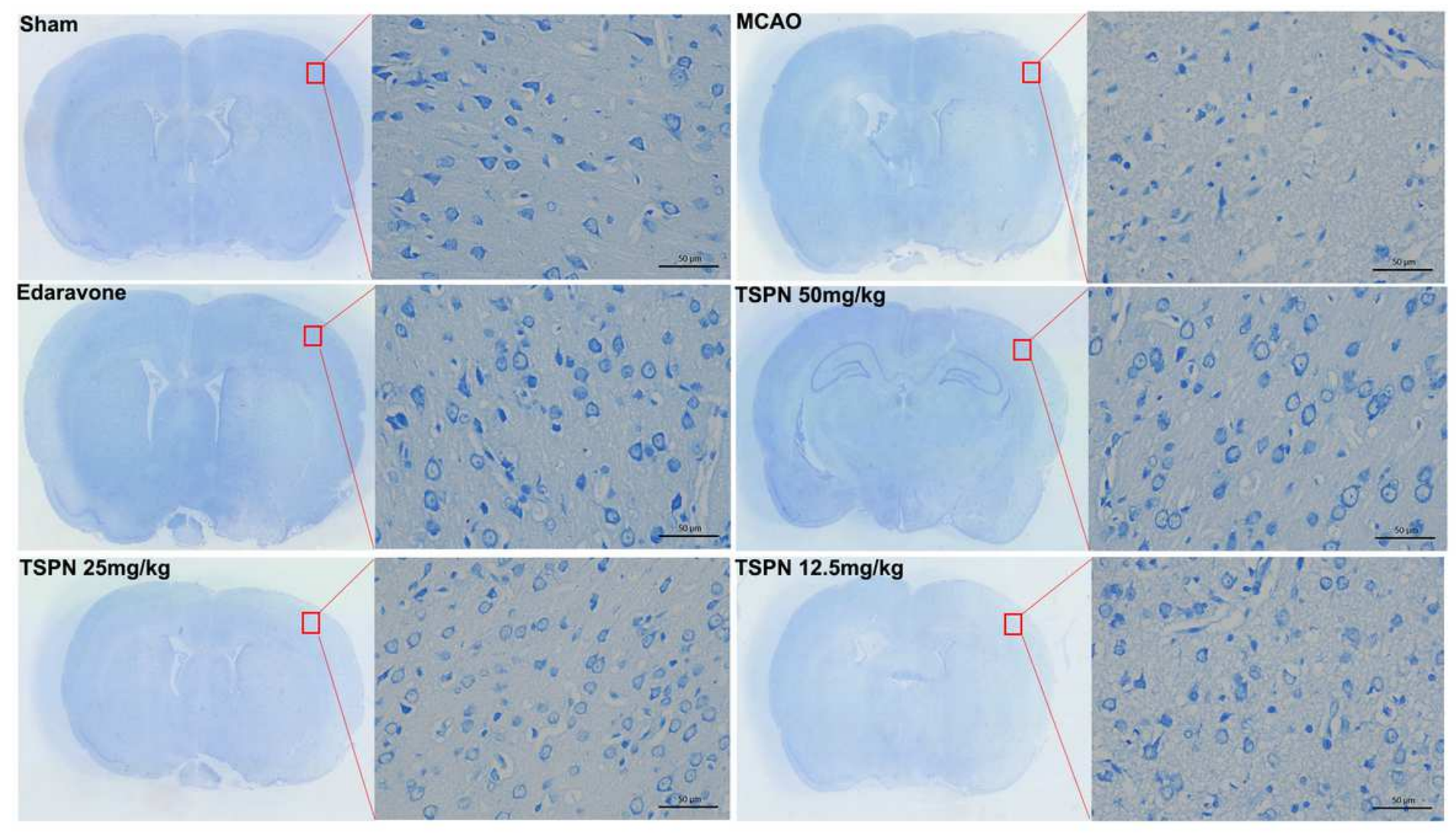

\section{Figure 8}

Nissl staining of ischemic penumbra (400x, scale bar $=50 \mu \mathrm{m}, \mathrm{n}=7)$ There was a big difference in morphology between normal neurons and abnormal neurons. The former had normal morphology, clear nucleolus, abundant cytoplasm and intact structure. The latter had shrinkage and deep staining. 


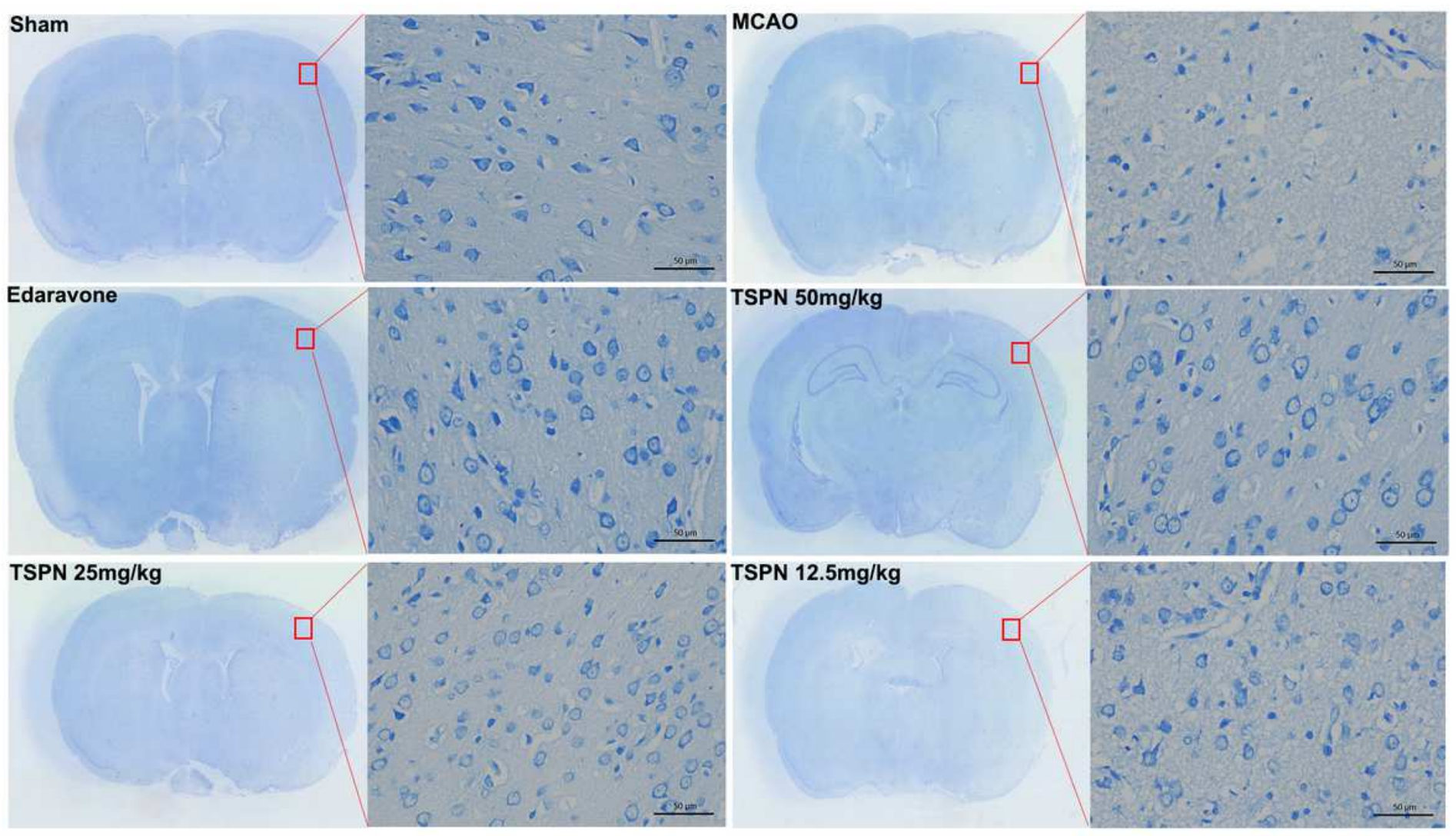

\section{Figure 8}

Nissl staining of ischemic penumbra (400x, scale bar $=50 \mu \mathrm{m}, \mathrm{n}=7)$ There was a big difference in morphology between normal neurons and abnormal neurons. The former had normal morphology, clear nucleolus, abundant cytoplasm and intact structure. The latter had shrinkage and deep staining.

a

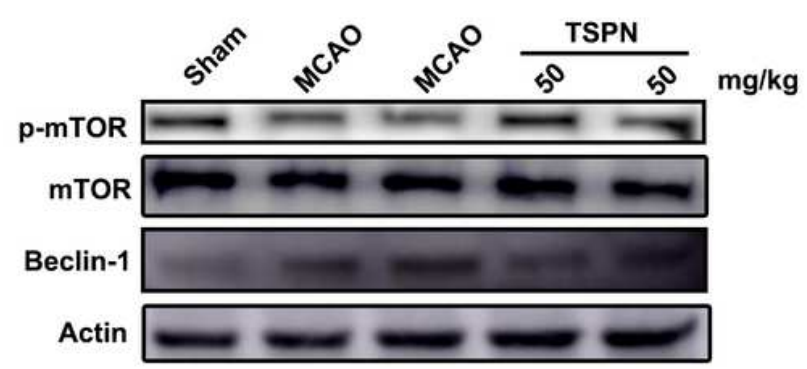

b

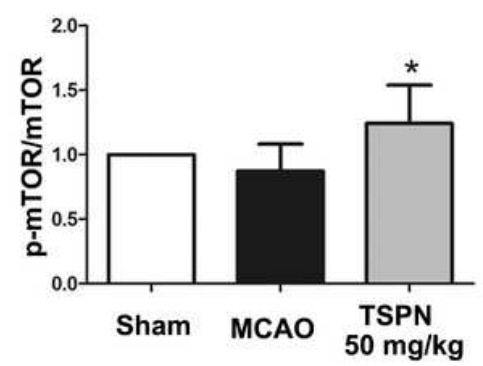

C

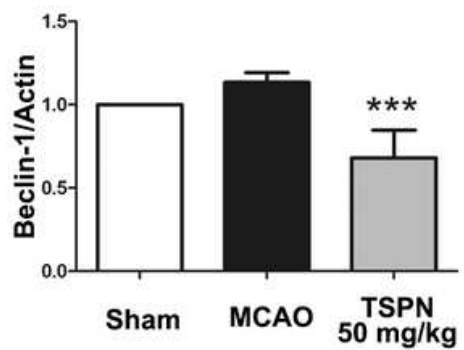

\section{Figure 9}

Modulation of the protein levels of mTOR, p-mTOR, and Beclin-1 in ischemic penumbra. (a) Representative bands of mTOR, p-mTOR, and Beclin-1 detected by Western blot analysis. (b-c) Quantification of expression levels of p-mTOR (b) and Beclin-1(c). ${ }^{*} P<0.05, * \star \star P<0.001$ vs MCAO group, $\mathrm{n}=4$. The data are expressed as means \pm SD. 
a

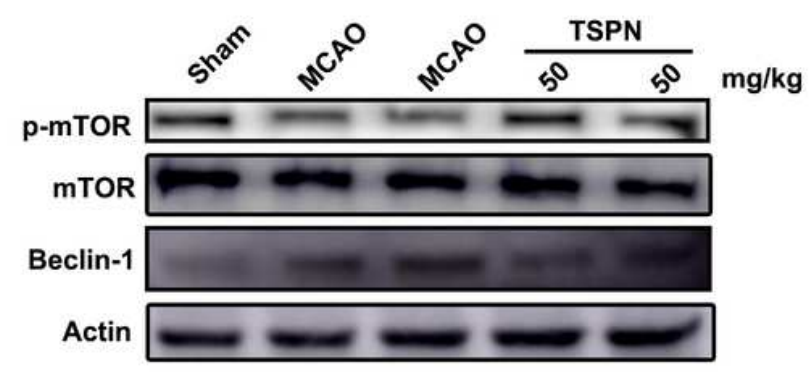

b

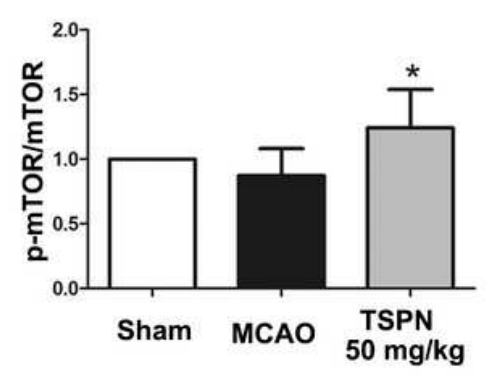

c

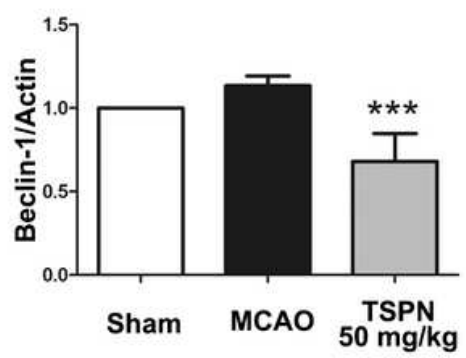

\section{Figure 9}

Modulation of the protein levels of mTOR, p-mTOR, and Beclin-1 in ischemic penumbra. (a) Representative bands of mTOR, p-mTOR, and Beclin- 1 detected by Western blot analysis. (b-c) Quantification of expression levels of p-mTOR (b) and Beclin-1(c). ${ }^{*} P<0.05, * \star * P<0.001$ vs MCAO group, $\mathrm{n}=4$. The data are expressed as means \pm SD.

a

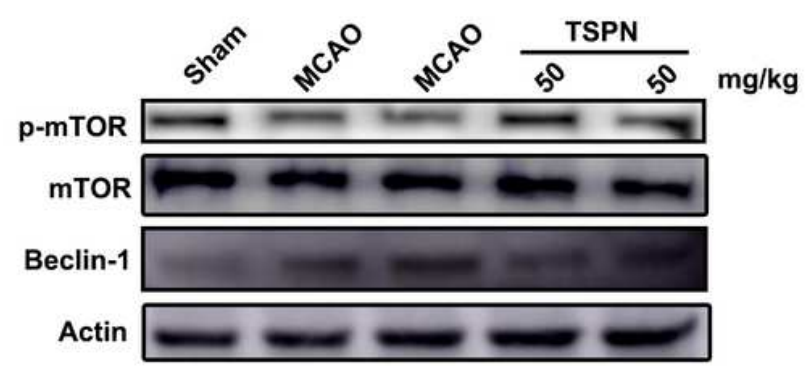

b

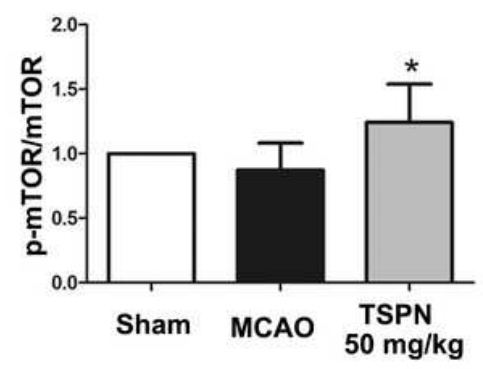

C

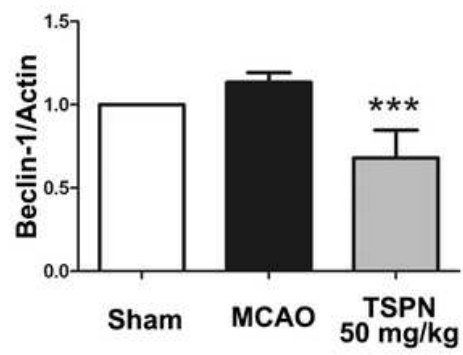

Figure 9

Modulation of the protein levels of mTOR, p-mTOR, and Beclin-1 in ischemic penumbra. (a) Representative bands of mTOR, p-mTOR, and Beclin- 1 detected by Western blot analysis. (b-c) Quantification of expression levels of p-mTOR (b) and Beclin-1(c). ${ }^{*} \mathrm{P}<0.05$, ${ }^{* \star *} \mathrm{P}<0.001$ vs MCAO group, $\mathrm{n}=4$. The data are expressed as means \pm SD. 
a

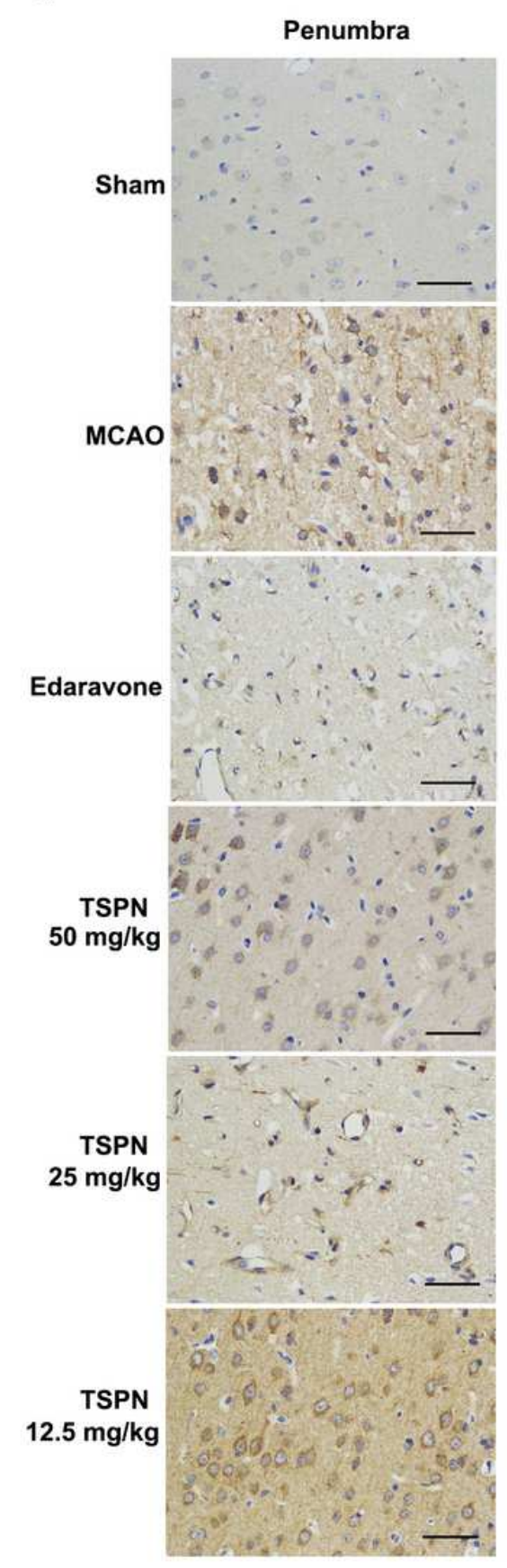

b

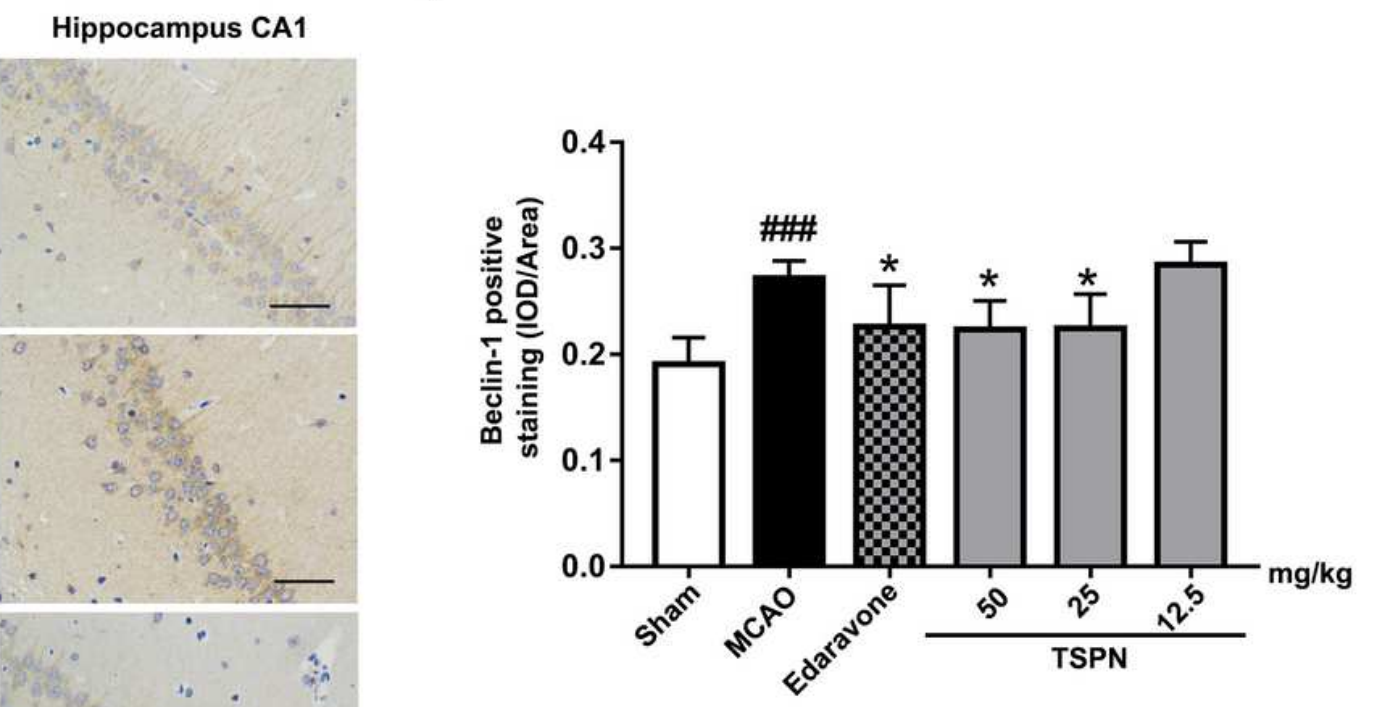

c

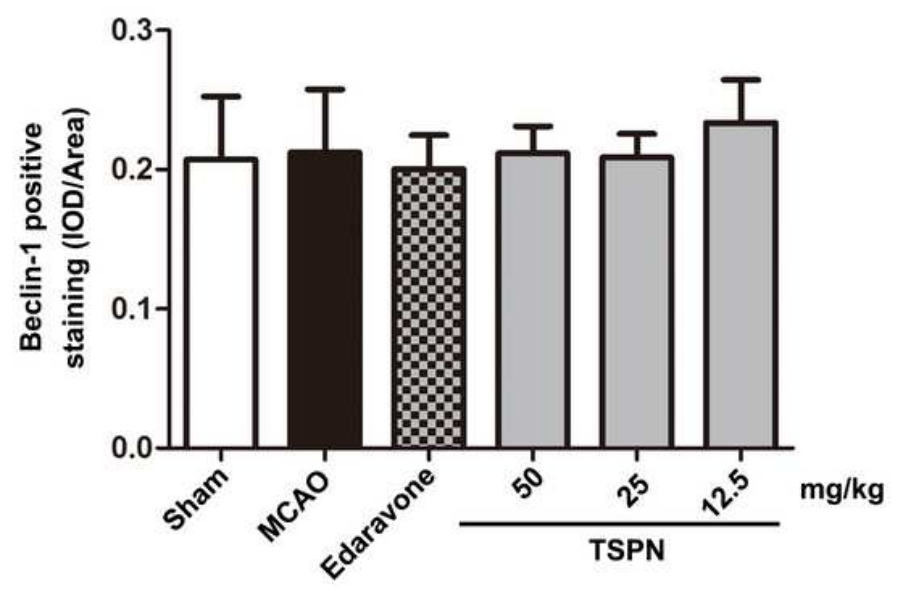

Figure 10

Immunohistochemistry was used to detect the expression of Beclin-1 in ischemic penumbra and hippocampus CA1 area of MCAO rats. (a) The average optical densities of Beclin-1 in ischemic penumbra (b) and hippocampal CA1 region (c) were analyzed using ImageJ (400 x, scale bar = $50 \mu \mathrm{m})$. \#\#\# < 0.001 vs Sham group, * $\mathrm{P}<0.05$ vs MCAO group, $\mathrm{n}=6$. The data are expressed as means \pm SD. 
a

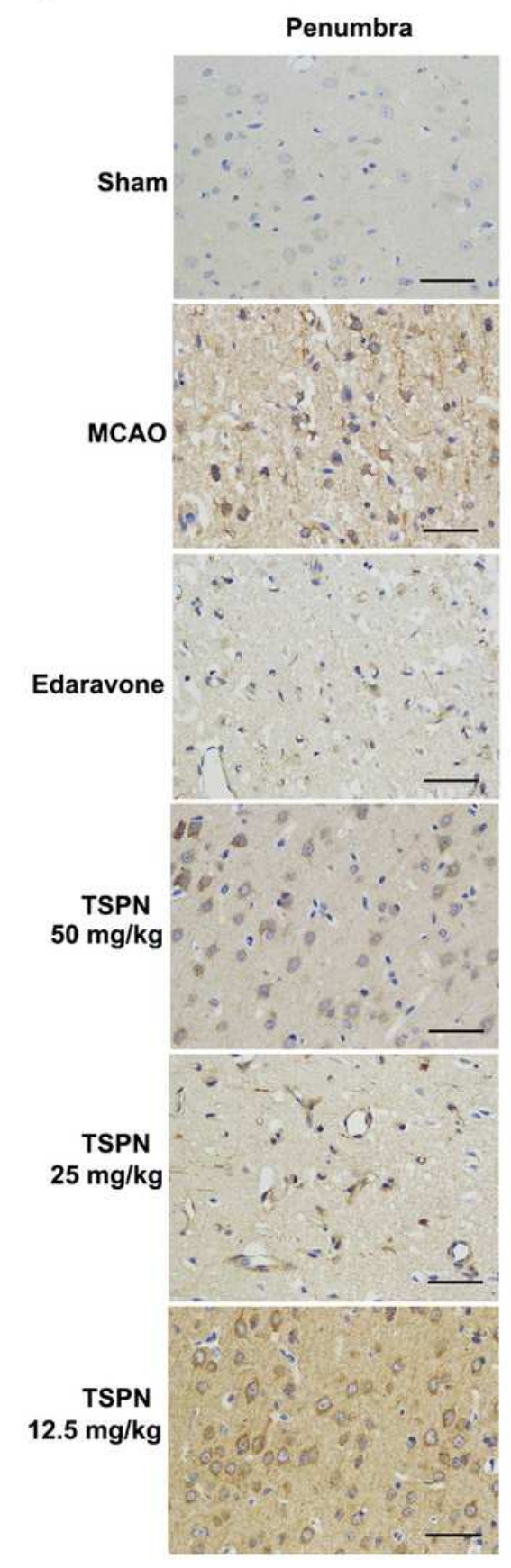

b

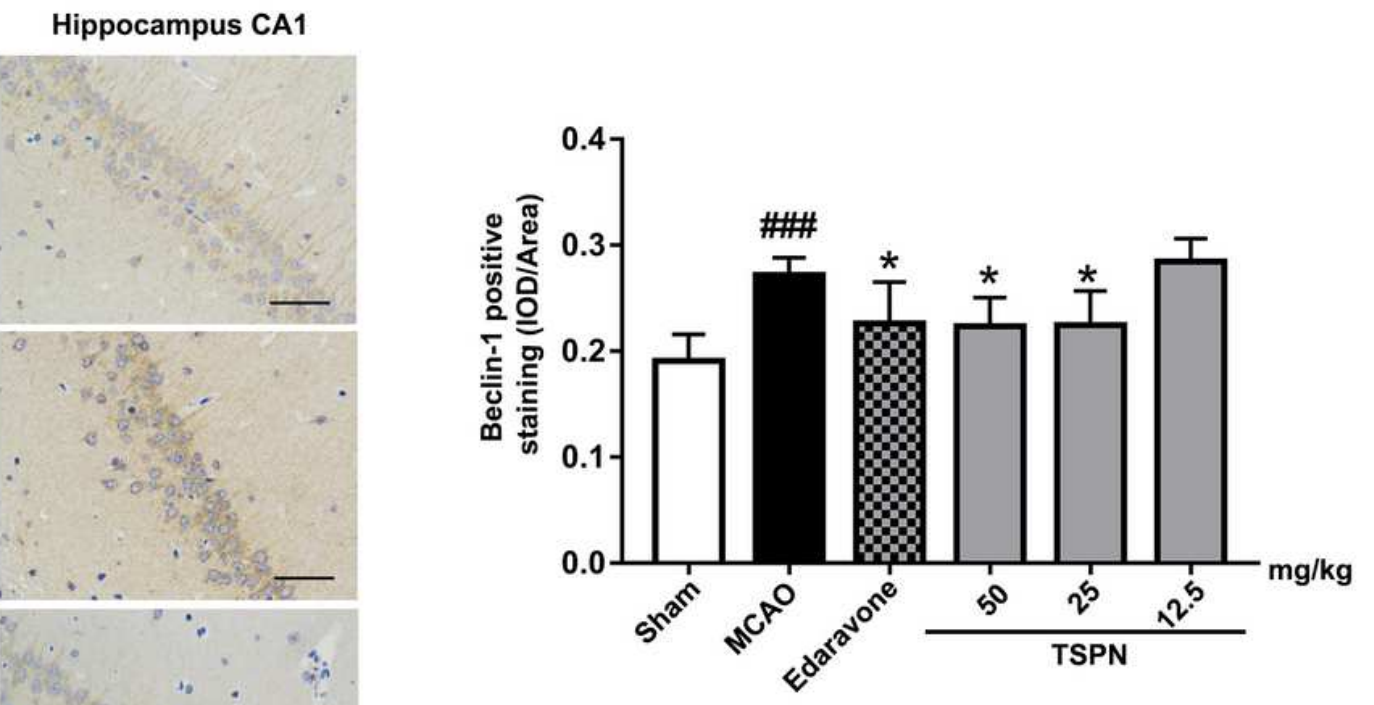

C

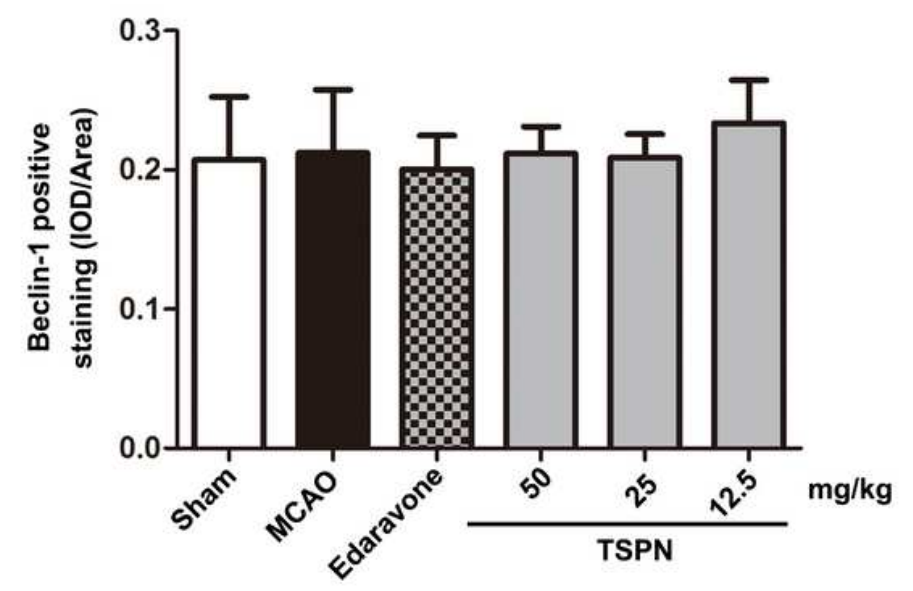

Figure 10

Immunohistochemistry was used to detect the expression of Beclin-1 in ischemic penumbra and hippocampus CA1 area of MCAO rats. (a) The average optical densities of Beclin-1 in ischemic penumbra (b) and hippocampal CA1 region (c) were analyzed using ImageJ (400 x, scale bar = $50 \mu \mathrm{m})$. \#\#\# < 0.001 vs Sham group, * $\mathrm{P}<0.05$ vs MCAO group, $\mathrm{n}=6$. The data are expressed as means \pm SD. 
a

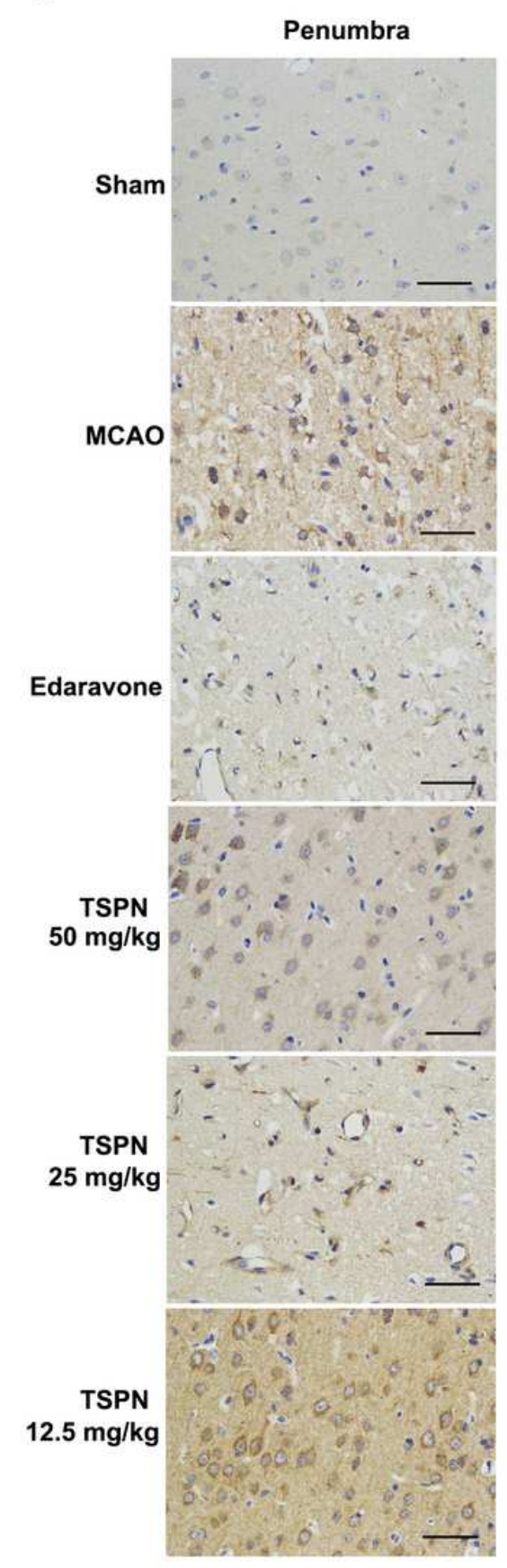

b

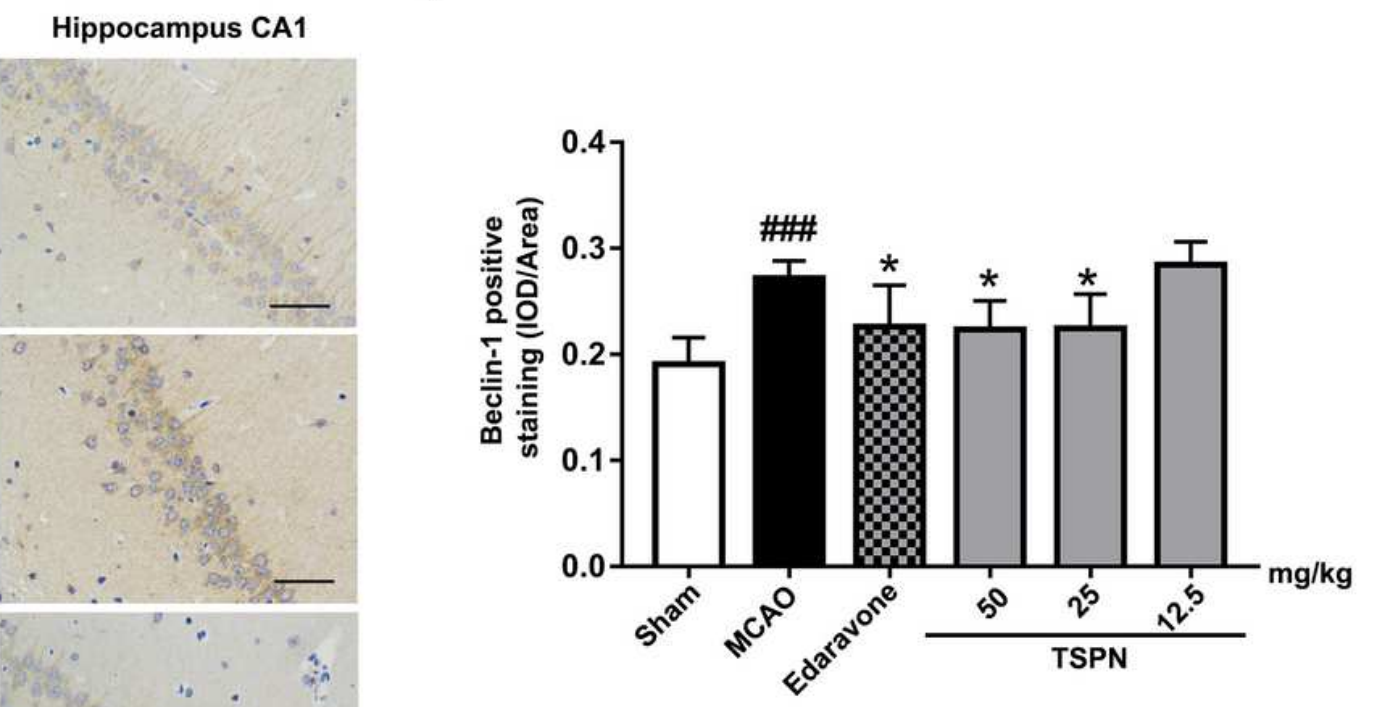

c

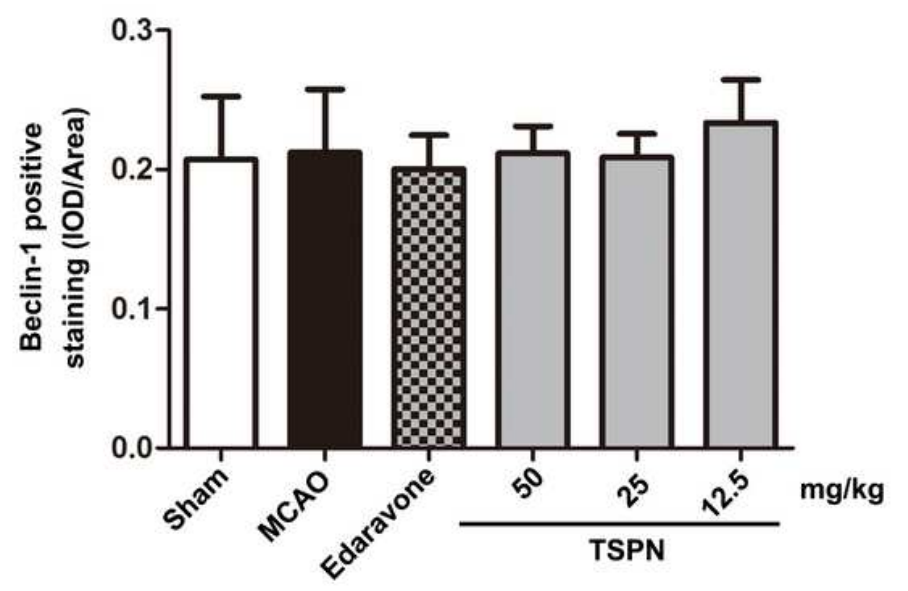

Figure 10

Immunohistochemistry was used to detect the expression of Beclin-1 in ischemic penumbra and hippocampus CA1 area of MCAO rats. (a) The average optical densities of Beclin-1 in ischemic penumbra (b) and hippocampal CA1 region (c) were analyzed using ImageJ (400 x, scale bar = $50 \mu \mathrm{m})$. \#\#\# < 0.001 vs Sham group, * $\mathrm{P}<0.05$ vs MCAO group, $\mathrm{n}=6$. The data are expressed as means \pm SD. 


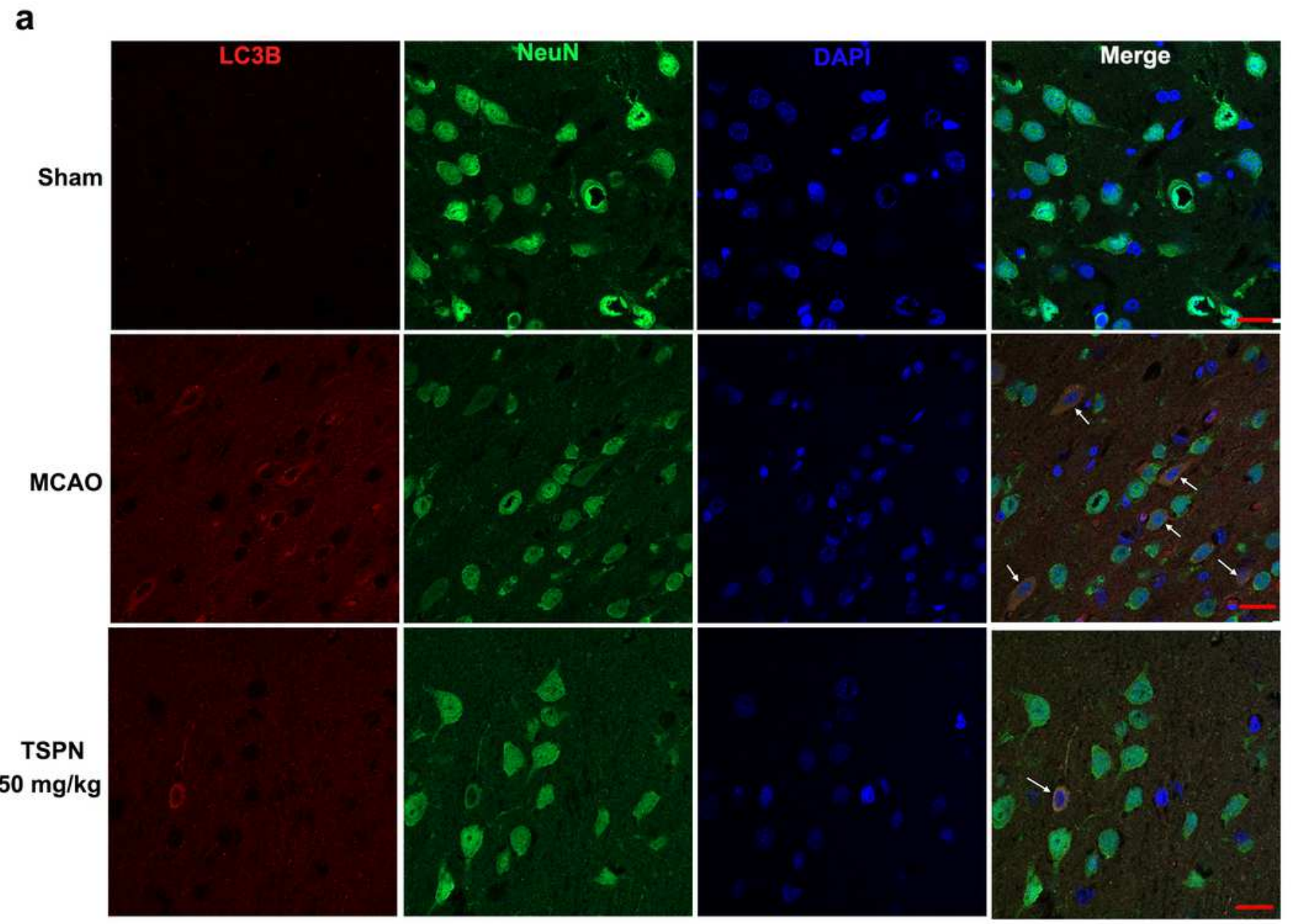

b

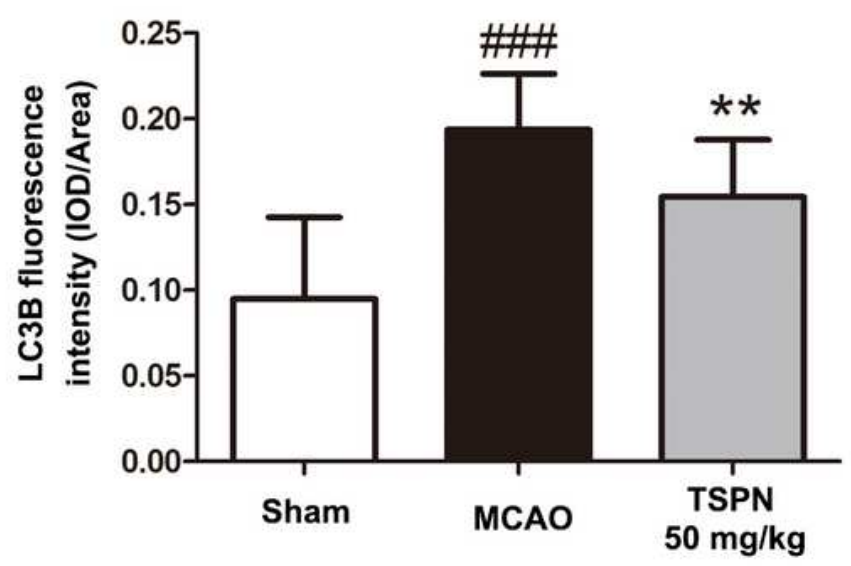

Figure 11

TSPN inhibit expression of LC3B in ischemic penumbra of MCAO rats. (a) Expressions of LC3B were identified by immunofluorescence. (b) The fluorescence intensity of LC3B was analyzed using ImageJ $(40 \times$ objective, scale bar $=20 \mu \mathrm{m})$. \#\#\#P $<0.001$ vs Sham group, $* \star P<0.01$ vs MCAO group, $n=4$. The data are expressed as means \pm SD. 


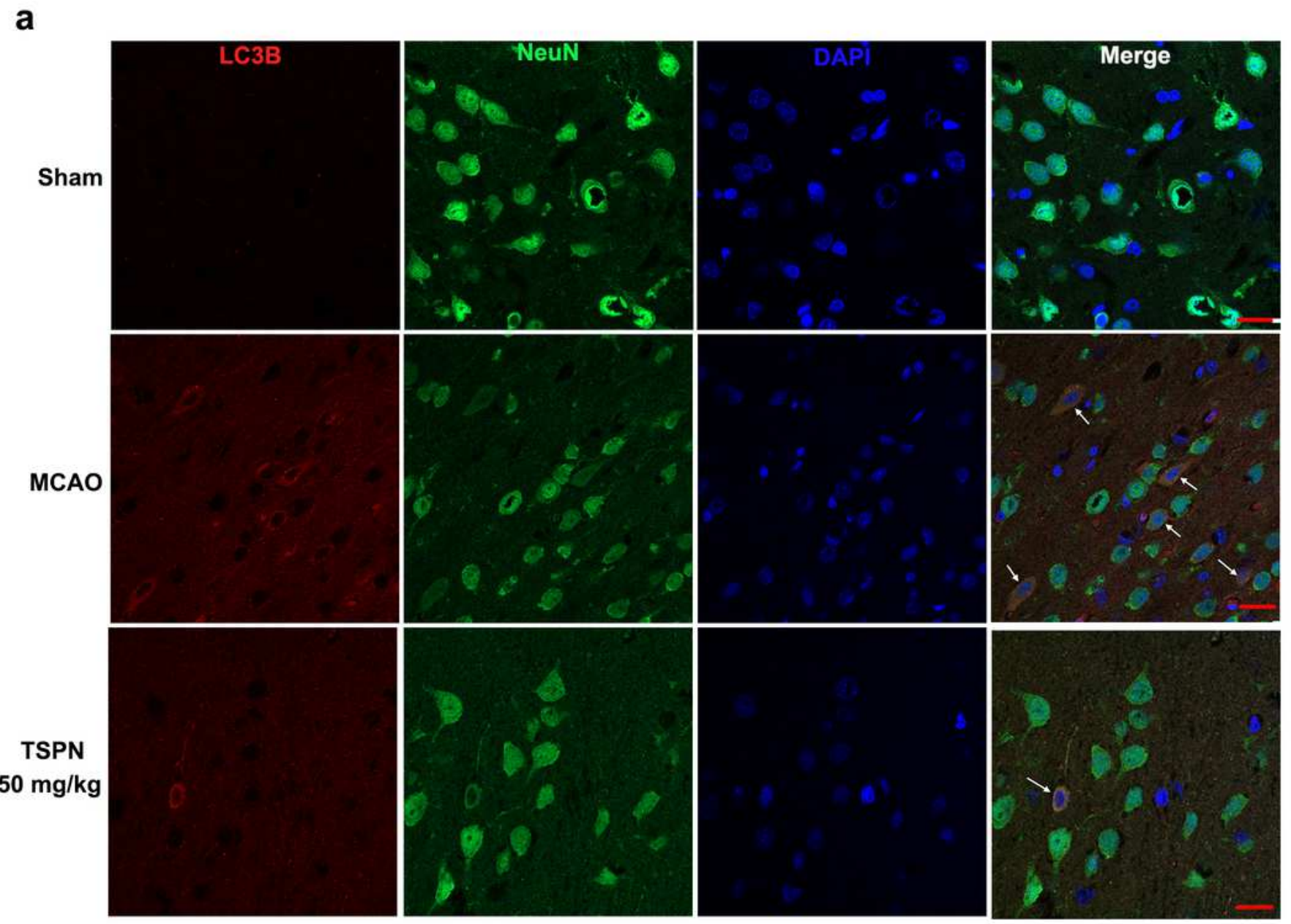

b

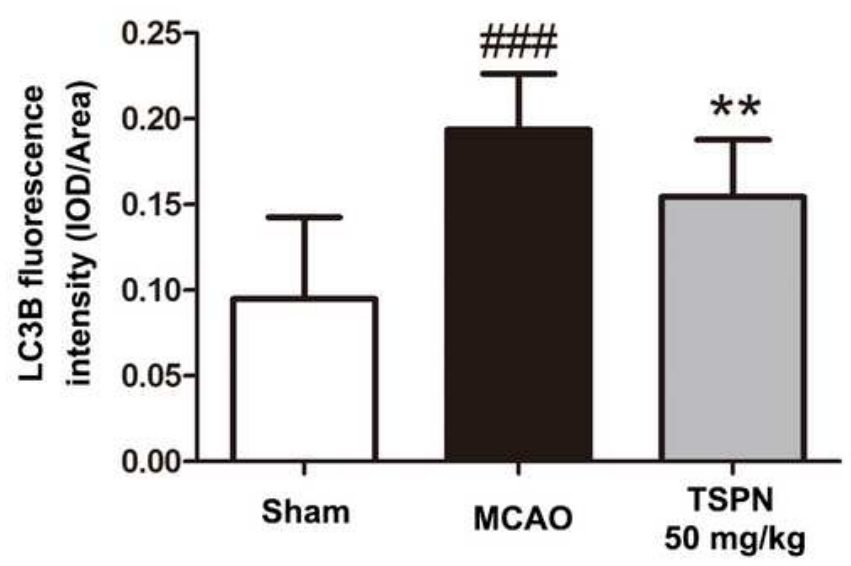

Figure 11

TSPN inhibit expression of LC3B in ischemic penumbra of MCAO rats. (a) Expressions of LC3B were identified by immunofluorescence. (b) The fluorescence intensity of LC3B was analyzed using ImageJ $(40 \times$ objective, scale bar $=20 \mu \mathrm{m})$. \#\#\#P $<0.001$ vs Sham group, $* \star P<0.01$ vs MCAO group, $n=4$. The data are expressed as means \pm SD. 


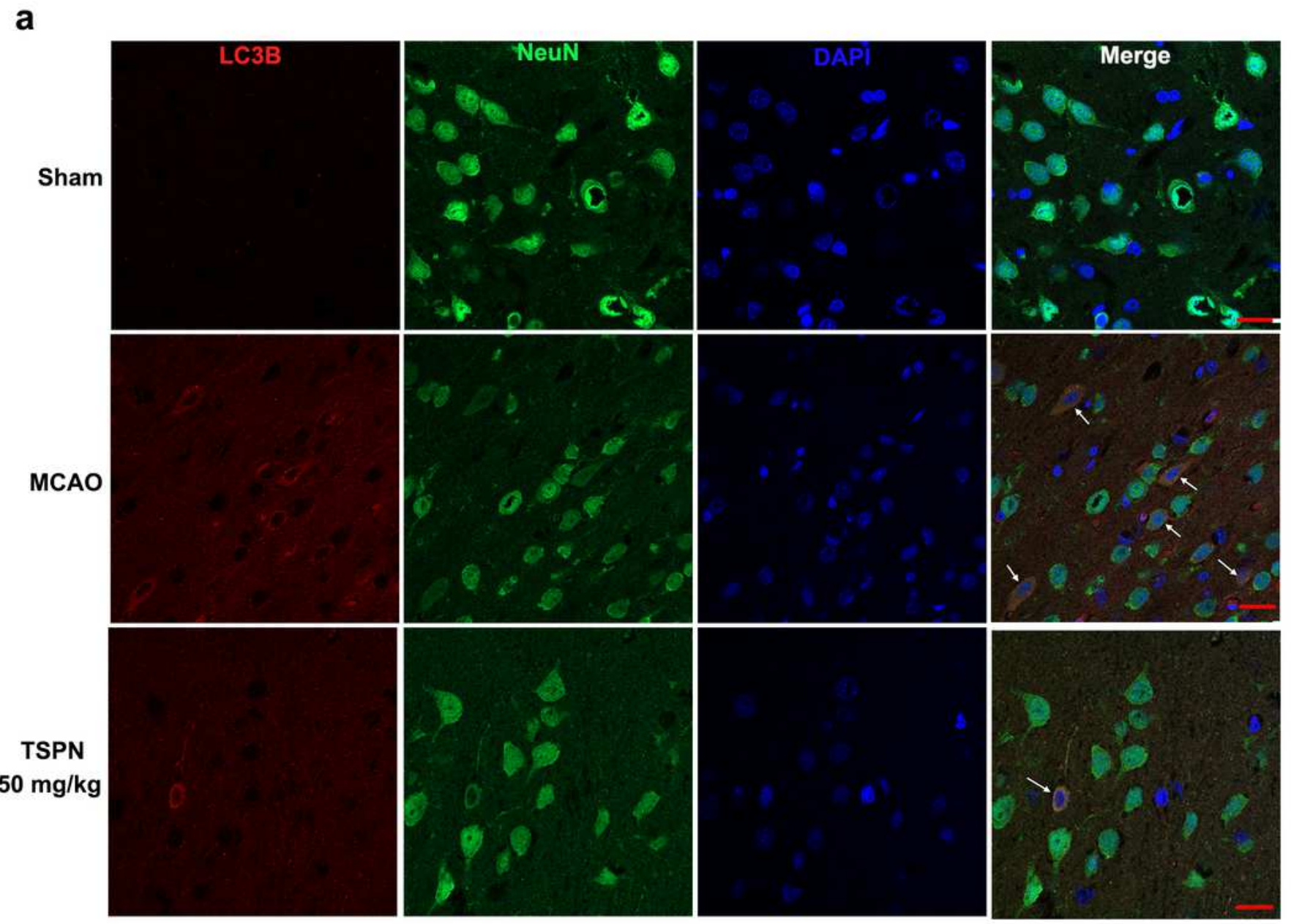

b

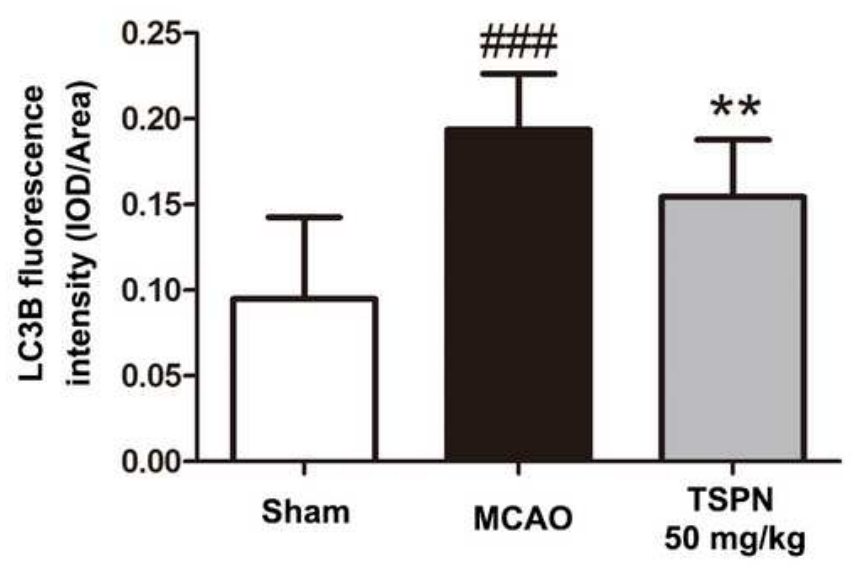

Figure 11

TSPN inhibit expression of LC3B in ischemic penumbra of MCAO rats. (a) Expressions of LC3B were identified by immunofluorescence. (b) The fluorescence intensity of LC3B was analyzed using ImageJ $(40 \times$ objective, scale bar $=20 \mu \mathrm{m})$. \#\#\#P $<0.001$ vs Sham group, $* \star P<0.01$ vs MCAO group, $n=4$. The data are expressed as means \pm SD. 